\author{
Accelerator Development Department \\ Accelerator Physics Division \\ BROOKHAVEN NATIONAL LABORATORY \\ Associated Universities, Inc. \\ Upton, NY 11973
}

Accelerator Physics Technical Note No. 40

\title{
Long Term Tracking with PATRIS \\ Part 2: Aberration Effects
}

J. Milutinovic and A.G. Ruggiero

April 1992 


\title{
Long Term Tracking with PATRIS Part 2: Aberration Effects
}

\author{
J.Milutinovic and A.G.Ruggiero \\ Accelerator Development Department \\ Brookhaven National Laboratory \\ Upton, NY 11973
}

\section{Introduction}

We continue our tracking studies of the RHICAGR lattice ${ }^{1}$, beyond those limited to determination of physical and dynamic aperture only ${ }^{2}$. In particular, we explore tune dependence on the amplitude, by examining Fourier spectrum of betatron oscillations. This spectrum was found to be very rich for some directions in the $\left(\epsilon_{\mathrm{X}}, \epsilon_{\mathrm{y}}\right)$ plane and displayed an anomalous secondary peak, whose height for certain values of the initial particle amplitude, and at some but not all locations in the lattice, exceeded the height of the primary peak. The position of the latter is customarily interpreted as the tune value at that particular amplitude. This prompted us to examine the phase space of the tracked particles in more detail. By examining the Floquet coordinates of the particles at the lattice bottleneck $\mathrm{BC} 2$, we were able to see a 5 -th integer resonance along $X \neq 0, Y=0$ direction, a 3-rd integer resonance for $X=0, Y \neq 0$ initial coordinates, and a typical emittance exchange for $X=Y$ initial direction. We also tried to determine how particles were lost during our exploration of the dynamic aperture. Once again, our study demonstrates the importance of exploring the behavior of particles for various initial conditions, which significantly differ from each other. 


\section{Physical/Dynamic Aperture Search - A Brief Summary}

In this section we briefly summarize our method of physical/dynamic aperture search and its basic results ${ }^{2}$. Using PATRIS ${ }^{3}$, we track $N$ particles in the error-free RHICAGR lattice ${ }^{1}$, which contains chromaticity correcting sextupoles as the only type of nonlinearities. We choose 1000 turns in all cases. We also choose to track $N=26$ particles at a time. This number was chosen since $N-1=25$ enabled us to deal with a convenient rational subdivision of the interval on the emittance axis, since $1 / 25=0.04$. We could have chosen a smaller number of particles to track, but this large number ensured a rapid convergence of our searching procedure and minimized the danger that dynamic aperture might be overestimated by having overlooked some particles which are lost even though their initial amplitudes are slightly smaller than the amplitudes of some particles that survived the tracking, a phenomenon typically occurring in computer tracking with nonlinearities. In our study this appeared frequently in the pure $Y$ initial direction and for $\delta=-0.4 \%$. Occasionally, it was observed at other $\delta$ values and in the $X=Y$ and $X=-Y$ initial direction. In the last case, this behavior was so pronounced that our automated searching procedure overestimated the dynamic aperture and the latter had to be recomputed by a manual adjustment of PATRIS input files. This case will be briefly discussed later in this note. However, we did not see this phenomenon occurring frequently in the pure $X$ direction. The latter proves that this behavior, caused by the presence of nonlinearities, becomes pronounced as a result of coupling, which is absent as long as the condition $Y=0$ is strictly satisfied, and this is maintained by the equations of motion for the case of an error-free lattice, if initially $Y=0$ and $Y^{\prime}=0$.

The aperture scanning procedure now goes as follows. We choose a direction in the $\left(\epsilon_{\mathrm{X}}, \epsilon_{\mathrm{y}}\right)$ plane, as described in Ref.[2], and launch $N=26$ particles, which all start with $X^{\prime}=Y^{\prime}=0$, and which are uniformly distributed between 0 and some maximum values $\epsilon_{\mathrm{X}}$ and $\epsilon_{\mathrm{y}}$. The last two are chosen to be big enough so that at least some of the particles will be lost. The code then tracked these particles for 1000 turns and at the end it determined the particle with the smallest values of $\left(\epsilon_{\mathrm{x}}, \epsilon_{\mathrm{y}}\right)$ that was lost. Some particles that survived the tracking despite their greater initial amplitudes, a typical phenomenon in the presence of nonlinear coupling, were dismissed and not counted as surviving. The "last survivor" 
was the surviving particle with the greatest initial amplitude but still smaller than the least initial amplitude among the lost particles. This emittance interval between the last survivor and the first lost particle was now divided by $N-1=25$, and another set of $N=26$ particles were launched. This iterative procedure was repeated until the "emittance bounds error" defined to be:

$$
\Delta \epsilon=\left[\left(\epsilon_{x}^{\mathrm{LOST}}-\epsilon_{x}^{\mathrm{SURV}}\right)^{2}+\left(\epsilon_{y}^{\mathrm{LOST}}-\epsilon_{y}^{\mathrm{SURV}}\right)^{2}\right]^{1 / 2}
$$

dropped below a predetermined value $\Delta \epsilon_{M}$. In our case this was taken to be $\Delta \epsilon_{M}=0.1 \pi$ $\mathrm{mm} \cdot \mathrm{mrad}$ and was reached in just two iterations. A smaller number of tracked particles would require more iterations for a fixed $\Delta \epsilon_{M}$. At the end of the procedure the code printed the so-called "final emittance bounds"

$$
\left[\epsilon_{x}^{\mathrm{SURV}}, \epsilon_{x}^{\mathrm{LOST}}\right], \quad\left[\epsilon_{y}^{\mathrm{SURV}}, \epsilon_{y}^{\mathrm{LOST}}\right]
$$

which meant that the limiting case, i.e. the border between being reasonably assured of surviving and being empirically found to be lost, is lying between these intervals. For each lost particle, PATRIS also printed the location of the loss. This procedure enabled us to determine physical/dynamic aperture for each direction in the $\left(\epsilon_{\mathrm{x}}, \epsilon_{\mathrm{y}}\right)$ plane very rapidly and with high confidence.

The criteria of particle loss were as follows. For physical aperture determination, with or without sextupoles, a particle was considered lost if its distance from the origin in any direction exceeded the available magnet aperture at the lattice bottleneck, i.e. at the magnet $\mathrm{BC} 2$, that limits the lattice acceptance determined by the code to be $5.08 \pi$ $\mathrm{mm} \cdot \mathrm{mrad}$. On the other hand, in the dynamic aperture search a particle was considered lost if its distance from the origin exceeded $1000 \mathrm{~mm}$, at any checking point in the lattice. The checking points were the previously mentioned bottlenecks, all of the sextupoles and the middle point of each quadrupole.

The aperture was determined for momentum deviation values $\delta=-0.6,-0.4,-0.2$, $0.0,+0.2,+0.4,+0.6$ percent. The results are reported in our previous note ${ }^{2}$. For the sake of completeness, we give here only the results for $\delta=0.0$, since all the analysis in this note is referred to the on-momentum case. The final emittance bounds are given in Table 1. An improved application of the method described above gave results which are more 
stringent than those shown in Ref.[2], especially for directions \# 2 and \# 4 (and of course \# 6 and \# 8, by mid-plane symmetry).

\section{Search for Tune Dependence on the Amplitude and Subsequent Analysis}

In addition to various analytic means, particle tracking is one of the methods of determining the dependence of the tune on the particle amplitude. The tune is taken to be the position of the highest peak in the Fourier spectrum of betatron oscillations.

In order to get reliable functional dependence, we launched particles in five out of eight directions described in our previous note. The remaining three directions \# 6, \# 7 and \# 8, corresponding to negative values of the initial vertical amplitude, were not examined since the lattice exhibits a perfect mid-plane symmetry in the absence of errors, and these characteristics of the lattice were clearly manifested in our previous tracking studies of physical/dynamic aperture. We launched $N=26$ equally spaced particles in the emittance intervals of $1 \pi \mathrm{mm} \cdot \mathrm{mrad}$ and this means that we examined amplitudes corresponding to emittances spaced by $1 / 25=0.04 \pi \mathrm{mm} \cdot \mathrm{mrad}$ intervals, starting from 0 and ending at the edge of the physical aperture in the presence of sextupoles. This provided typically $\sim 100$ points - values of tune between the particle with zero emittance and the edge of the physical aperture.

To understand better the results of computing tune versus emittance, by particle tracking, we also generated phase space diagrams of various types. We were particularly urged to go into this kind of analysis by puzzling results for the direction \# 3 (initial displacement in pure $Y$ direction). In general, we generated phase space diagrams both at the starting point, i.e. in the middle of a defocusing quad at the beginning of the arc, and at the lattice bottleneck $\mathrm{BC} 2$. We generated plots both in the standard transverse coordinates $\left(X, X^{\prime}, Y, Y^{\prime}\right)$ and in the Floquet coordinates which are related to the former ones by the relations:

$$
\begin{aligned}
& u=X / \sqrt{\beta_{x}}, \quad u^{\prime}=\left(\alpha_{x} / \sqrt{\beta_{x}}\right) X+\sqrt{\beta_{x}} X^{\prime} \\
& v=Y / \sqrt{\beta_{y}}, \quad v^{\prime}=\left(\alpha_{y} / \sqrt{\beta_{y}}\right) Y+\sqrt{\beta_{y}} Y^{\prime}
\end{aligned}
$$


Note that the Floquet coordinates of a particle satisfy the relations:

$$
u^{2}+u^{\prime 2}=\epsilon_{x}, \quad v^{2}+v^{\prime 2}=\epsilon_{y}
$$

and that in the absence of coupling the quantities $\epsilon_{\mathrm{x}}$ and $\epsilon_{\mathrm{y}}$ are separately invariant. All these phase space diagrams were generated by PATRIS, which had been equipped with the necessary routines to extract the phase space coordinates of a particle at any desired location in the lattice, and to transform them into the Floquet representation before plotting, if so desired.

For the sake of completeness and for further reference we mention the linearized equations of motion for a particle in an error-free lattice, modified in the presence of sextupoles by thin lens perturbations (kicks) at their right hand sides:

$$
\begin{aligned}
X^{\prime \prime}+\left(\frac{1}{\rho^{2}}-K\right) X+\frac{\delta}{\rho} & =S\left(X^{2}-Y^{2}\right) / 2 \\
Y^{\prime \prime}+K Y & =-S X Y .
\end{aligned}
$$

To make our discussion complete and to have some comparison of our tune dependence on emittance, obtained by tracking, with analytic results, we also present the results from $\mathrm{MAD}^{4}$ and SYNCH${ }^{5}$. Linear perturbation theory ${ }^{6}$ gives the following expressions for tuneshifts experienced by the particles with nonzero emittances $\epsilon_{\mathrm{x}}$ and $\epsilon_{\mathrm{y}}$

$$
\begin{aligned}
& \Delta \nu_{x}=a \epsilon_{x}+b \epsilon_{y} \\
& \Delta \nu_{y}=b \epsilon_{x}+c \epsilon_{y} .
\end{aligned}
$$

Analytic calculations implemented in MAD and SYNCH fully agree on the values of these coefficients, which are given below

$$
\begin{aligned}
& a=-2.362 \mathrm{E}+03 \\
& b=-1.889 \mathrm{E}+03 \\
& c=1.378 \mathrm{E}+03
\end{aligned}
$$

and which will be compared with our tracking results at small values of emittances, i.e. in the regions where the linear approach is presumed valid.

We will now present the results of our analysis for the five directions in the $\left(\epsilon_{\mathrm{x}}, \epsilon_{\mathrm{y}}\right)$ plane. Directions \# 1 and \# 5 display the same qualitative behavior and the same is true for \# 2 and \# 4. Consequently, we group them together while direction \# 3 stands alone. 


\section{Results for Directions \# 1 and \# 5}

For these two directions the initial conditions $Y=0$ and $Y^{\prime}=0$ are fulfilled. Furthermore, they are preserved throughout the entire particle motion, as seen from the equations of motion (4) and (5), for an error-free lattice that we simulate. Therefore, the vertical motion never get excited. We present phase space plots for direction \# 1 in Figures 1 - 5 . Depending on the particle emittance the phase space diagrams are:

1. An ellipse (a circle in Floquet variables) for small values of $\epsilon_{\mathrm{X}}$, shown in Figure 1.

2. A pentagon for medium values of $\epsilon_{\mathrm{X}}$, shown in Figure 2 .

3. Five islands for large values of $\epsilon_{\mathrm{X}}$, shown in Figure 3 .

This proves the existence of a fifth-integer systematic resonance, excited by the sextupoles. We also show the behavior of the particles near the edge of the dynamic aperture. Figure 4 shows the phase space diagram of a particle at the very edge (within our tracking accuracy, of course) of the dynamic aperture, while Figure 5 shows a particle whose emittance is barely beyond the edge of the dynamic aperture. It makes just 209 turns instead of 1000. Direction \# 5 is very similar to direction \# 1 , so all the comments going with Figures 1 - 5 also apply to Figures 6 - 10. Note that the dynamic aperture for direction \# 5 is just slightly smaller than the same quantity for direction \# 1, as seen from Table 1. That explains why the particle \# 22 behaves as "the last survivor" in Figure 4, while at the same time it appears to be "the first lost particle" in Figure 10. A closer examination of the trajectories in Figures 4 and 9 shows that they behave "regularly" with a "closed" pattern, whereas the trajectories in Figures 5 and 10 are clearly of "chaotic" behavior and reach larger amplitudes. Thus the dynamic aperture seems to be born by some chaoticity at large amplitudes, induced solely by the presence of sextupoles.

Tune dependence on the emittance is shown in Figure 11 for direction \# 1 and Figure 12 for direction \# 5. In both cases $\nu_{x}$ is a monotonically decreasing function of $\epsilon_{\mathrm{x}}$. The solid straight line in each case represents the results of analytic evaluations on the basis of linear approximation by MAD and SYNCH, which fully agree with one another. The hollow circles represent the results obtained by tracking with PATRIS. The tune is descending in a stepwise manner, which simply reflects the fact that FFT routine in PATRIS evaluates the tune to the accuracy of $1 /$ (No. of turns) $=1 / 1000=0.001$, hence the changes are 
always abrupt, by an increment of 0.001 . An increased number of tracking turns would also augment the number of these "stairs" per an emittance interval and make them shorter and narrower. Finally, a sufficient increase would remove this "staircase" picture and arrange the circles along a curve. This functional dependence is (within our tracking accuracy and with 1000 turns) the same for both directions in the emittance interval $[0.0,2.12]$ $\pi \mathrm{mm} \cdot \mathrm{mrad}$. In this interval the agreement between tracking based results, and MAD and SYNCH on the other hand is good, while for the smaller emittances $\leq 1 \pi \mathrm{mm} \cdot \mathrm{mrad}$ it is even better. This shows that the three codes agree in the region where the dependence is predominantly linear, but fail to agree for larger emittances where PATRIS picks up the nonlinear contributions, as obvious from the shapes of the curves that would interpolate the sequences of circles in Figures 11 and 12 . Beyond $2.12 \pi \mathrm{mm} \cdot \mathrm{mrad}$ there are very slight differences in tracking based results, apparently due to nonlinearities. Note that Figure 11 displays functional dependence of the tune until the emittance $4.36 \pi \mathrm{mm} \cdot \mathrm{mrad}$ is reached, while Figure 12 does the same up to $4.84 \pi \mathrm{mm} \cdot \mathrm{mrad}$. The difference comes from the fact that the physical aperture, unlike its dynamic counterpart, is slightly greater for direction \# 5 that for direction \# 1.

\section{Results for Directions \# 2 and \# 4}

These two directions are different from directions \# 1,3 and 5 in the magnitude of the effects of coupling. As we have mentioned, directions \# 1 and \# 5 effectively describe a one-dimensional nonlinear motion, owing to the fact that the vertical degree of freedom never gets excited. As far as direction \# 3 is concerned, the magnitude of coupling is not very great. The reason for this is the fact that for these initial conditions the horizontal degree of freedom gets excited only through the second order $Y^{2}$ perturbation on the RHS of Eq. (4), and this is small when compared to the first order effects, as we shall see later. On the other hand, for directions \# 2 and \# 4 the initial displacements $X$ and $Y$ are equal in magnitude, and Eq. (5) shows that the perturbation of the vertical degree of freedom is linear in the horizontal displacement $X$. Therefore, the two degrees of freedom strongly influence each other through their coupling.

This strong coupling produced some trouble during the procedure of determining the dynamic aperture. As we have mentioned in the introductory section, some particles were 
lost even though their initial amplitudes were slightly smaller than the amplitudes of some particles that did survive the tracking. There is a strong potential for overestimating the dynamic aperture in these circumstances, since a finite number of particles are spread over an emittance interval and some of these regions of instability may not be probed at all. A classical example is what we present in Table 2. In trying to generate illustrative graphs for direction \# 4, we launched $N=26$ particles in the emittance interval [2.82, $6.60] \pi \mathrm{mm} \cdot \mathrm{mrad}$. This means that the spacing between two adjacent particles was $(6.60$ - 2.82) $/ 25=0.15 \pi \mathrm{mm} \cdot \mathrm{mrad}$, which is not too rough a subdivision at all! Nevertheless, we see from the table that despite the fact that particles \# 2, 3, 4 and 6 were lost, all the particles between \# 7 and 17 survived and that covered the emittances between 3.73 and $5.24 \pi \mathrm{mm} \cdot \mathrm{mrad}$. Tables 3 and 4 represent the results of probing the subinterval [3.73, $5.24] \pi \mathrm{mm} \cdot \mathrm{mrad}$ with $N=26$ particles. The spacing is now reduced to $((5.24-3.73) / 25$ $=0.06 \pi \mathrm{mm} \cdot \mathrm{mrad}$. Now the fine structure starts appearing and we detect some of the lost particles in this interval, completely overlooked in the initial scanning. What is even more amazing is the difference between results in Table 3 and 4 . Table 3 represents the interval $[3.730,5.240] \pi \mathrm{mm} \cdot \mathrm{mrad}$ while Table 4 represents [3.730, 5.239] $\pi \mathrm{mm} \cdot \mathrm{mrad}$, which is really a minute change in the initial conditions of the 26 particles spread within the interval we explore. This explains why it was difficult to determine the dynamic aperture in this direction and why we first overestimated this quantity. Eventually we had to resort to a manual brute-force exploration to find out that the dynamic aperture for the on-momentum case stood at $2.318 \pi \mathrm{mm} \cdot \mathrm{mrad}$. It is worthwhile to mention that the smallest emittance of any lost particle that we observed was $2.323 \pi \mathrm{mm} \cdot \mathrm{mrad}$, while at the same time the largest emittance of any particle that survived 1000 turns stood at $7.056 \pi \mathrm{mm} \cdot \mathrm{mrad}$ !

These considerations have led us to conclude that methods used by many to determine the physical/dynamic aperture are faulty, at least for some initial conditions. They consist of initiating a search at larger amplitudes, then proceeding toward smaller amplitudes in small steps and finally stopping the procedure when the first "surviving" particle is detected. Contrary to somebody's expectation that this "survivor" marks the edge of the aperture, there is a richer structure that we have observed, typical of $X=Y$ and $X=-Y$ cases; the other cases with $X=0$ or $Y=0$ have shown a more regular, straightforward 
behavior. This is enough to caution interpretation of tracking results based on the "first look" approach.

If we generate phase space plots in the Floquet variables for emittances which are not too large (say $\leq 1 \pi \mathrm{mm} \cdot \mathrm{mrad}$ ), we obtain circles. They are shown in Figures 13 and 14 for direction \# 2 and Figures 15 and 16 for direction \# 4. These circles (phase space ellipses in ordinary variables $X, X^{\prime}, \dot{Y}, Y^{\prime}$ ) are now fairly thick. This indicates that each emittance is changing its value from turn to turn. This emittance exchange is a typical consequence of the coupling, provided by the chromaticity correcting sextupoles. To examine this, we plot the values:

$$
u^{2}+u^{\prime 2}=\epsilon_{x}, \quad v^{2}+v^{\prime 2}=\epsilon_{y}
$$

versus number of turns, for initial values $\epsilon_{\mathrm{x}}=\epsilon_{\mathrm{y}}=1$. We notice that the sum of the two emittances

$$
\epsilon_{x}+\epsilon_{y} \approx 2
$$

remains fairly constant, with only up to about 10 percent modulation. This situation is displayed in Figures 17 and 18 for the directions \# 2 and \# 4, respectively. In the figures we plot the two effective emittances and their sum, versus the number of turns. The exchange between the two emittances is obvious, as well as an approximate constancy of their sum, with up to 10 percent modulation. The horizontal emittance shows a greater average value, i.e. it usually expands away from its initial value $1.0 \mathrm{mmm} \cdot \mathrm{mrad}$, while the vertical emittance more often contracts. The latter property explains why the circles for the vertical Floquet variables have most of their interiors filled up (especially for direction \# 2.), while for the horizontal variables the circles remain hollow. When comparing direction \# 2 and \# 4 one also observes that the emittance exchange is somewhat more rapid for direction \# 4 and also that the average sum of the two effective emittances, unlike its counterpart in direction \# 2, has a tendency to fluctuate above its initial value $2.0 \pi \mathrm{mm} \cdot \mathrm{mrad}$. We also made the same exercise for medium range emittances of $1.8 \pi \mathrm{mm} \cdot \mathrm{mrad}$. The results are shown in Figures 19 and 20. All comments from the previous case apply here. We would only add that the emittance exchange proceeds at a rate which is about 1.8 times faster than the rate for the previous case of emittance being $1.0 \pi \mathrm{mm} \cdot \mathrm{mrad}$. Apparently, the coupling is more effective for larger initial emittances. 
We generate phase space plots for larger emittances too. As we increase them, the circles for the horizontal degree of freedom gradually change shape to become thickened pentagons, thereby revealing the presence of a fifth-integer resonance which we very clearly observed in directions \# 1 and \# 5. However, the vertical degrees of freedom produce phase space plots too smeared to reveal any obvious pentagonal structure. This is represented in Figures 21 and 22 for direction \# 2, and 23 and 24 for direction \# 4. The pentagons in the figures are obtained at emittance values close to the edge of the dynamic aperture. In Figures 21 and 22 the emittance is 2.52 and the dynamic aperture is 2.63, while in Figures 23 and 24 it is 2.24 versus $2.32 \pi \mathrm{mm}$. mrad. Within the available dynamic aperture, the pentagons do not display a tendency to break up to form islands. However, if we go slightly beyond the dynamic aperture and generate diagrams for stable particles (Figures 25 through 28), we see the beginning of the tendency to form islands, as obvious from Figures 25 and 27 . We also present phase space plots of unstable particles, lying just beyond the edge of the dynamic aperture, in Figures 29 through 32. It is clearly observed that the dynamic aperture is set by some chaotic behavior in the proximity of the fifth-order resonance islands.

Finally, in Figures 33 and 34, we present the results describing tune dependence on the emittance within the physical aperture. The tunes are monotonic functions of the emittance in the whole interval from 0 to the edge of the physical aperture (excluding the behavior of the vertical tune in a tiny interval around $\epsilon_{\mathrm{x}}=\epsilon_{\mathrm{y}}=0.5 \pi \mathrm{mm} \cdot \mathrm{mrad}$ ). For comparison we show also the analytical results from $\mathrm{SYNCH}$ and MAD. All the comments and clarifications made about Figures 11 and 12 apply here. However, the agreement between MAD and SYNCH, on the one hand, and PATRIS on the other, is poorer. The reason is obvious. Figures 11 and 12 represent essentially a one-dimensional nonlinear motion, with no coupling, whereas Figures 33 and 34 refer to a fully two-dimensional motion, with a fairly strong coupling, and as a result the disagreement begins at smaller emittance values than for directions \# 1 and \# 5 .

\section{Results for Direction \# 3}

This direction was the most puzzling one in our initial investigations of tune dependence on the emittance, and in fact prompted us to explore all of the directions in more detail, by 
generating the phase space diagrams in Floquet variables at the lattice bottleneck $\mathrm{BC} 2$. To see the cause of our puzzle, the reader should look at Table 5, which shows tune dependance on the emittance in the interval between 0 and $1 \pi \mathrm{mm} \cdot \mathrm{mrad}$. We should point out that, though initially $X=0$, according to Eq.(4) a horizontal motion nevertheless develops, with smaller amplitudes. PATRIS in turn, detecting the motion, tries the Fourier expansion and the measurement of the horizontal betatron tune.

These results were somewhat surprising, as the table indicates. Whereas the fractional part of the vertical tune displayed the expected monotonous functional dependence on the emittance, its horizontal counterpart behaved so in a limited interval around 0 and then suddenly dropped to the value $\nu_{x}=0.650$, for $\epsilon_{\mathrm{y}}=0.36 \pi \mathrm{mm} \cdot \mathrm{mrad}$. It stayed there, as we were increasing the emittance, until $\epsilon_{\mathrm{y}}=0.48 \pi \mathrm{mm} \cdot \mathrm{mrad}$ was reached, where it jumped back to its original value $\nu_{x}=0.825$, which happens to be the tune value for zero emittance, found analytically from the lattice transfer matrix. Another "anomalous" region was reached at $\epsilon_{\mathrm{y}}=0.80 \mathrm{\pi mm} \cdot \mathrm{mrad}$, while "normal" behavior was restored at $\epsilon_{\mathrm{y}}=0.92 \pi \mathrm{mm} \cdot \mathrm{mrad}$. Aside from these dips, the horizontal tune was monotonically decreasing with the emittance, whereas the vertical tune was monotonically increasing without any irregularities. Similar behavior was also displayed for larger values of $\epsilon_{\mathrm{y}}$, until the physical aperture $\epsilon_{\mathrm{y}}=4.6 \pi \mathrm{mm} \cdot \mathrm{mrad}$ is reached for this direction.

In Figures 35 and 36 we display the spectra of one particle from a "normal" region, i.e. having an emittance dependent tune continuously connected to the tune at zero amplitude, and one particle from an "anomalous" region, i.e. where a dip occurs. In both cases the $X$ direction is showing a great richness of the betatron oscillation frequency spectrum, but qualitatively the two spectra are not different from each other. In addition to the main peak in the $X$ direction, there are other pronounced peaks and we suspected that the secondary peak somehow prevailed over the primary one in a region which we call "anomalous".

As the next step, we generated phase space diagrams, at the starting point in the lattice, in the variables $\left(X, X^{\prime}, Y, Y^{\prime}\right)$. They had been made before we equipped PATRIS with routines to generate plots based on Floquet variables. (The very reason why we made this option available in PATRIS was our desire to better understand the whole puzzle about direction \# 3.) We display some of the graphs corresponding to the emittances in 
the interval $[0.0,1.0] \pi \mathrm{mm} \cdot \mathrm{mrad}$, which is also covered by Table 5 . The $\left(Y, Y^{\prime}\right)$ phase space diagrams throughout the whole interval are just fairly thin ellipses with steadily growing axes as the emittance increases. An example is shown in Figure 37. On the other hand, the $\left(X, X^{\prime}\right)$ phase space diagrams are very different. If plotted on the same scale as the $\left(Y, Y^{\prime}\right)$ quantities, they appear just as smeared blobs. This is because of the smallness of the horizontal motion, which is being generated entirely through the second order coupling, as seen from Eq.(4). However, if magnified by a factor 10, these blobs expand into clean triangular shapes which reveal the presence of a third-integer resonance. These diagrams are displayed in Figures 38 through 48. Figures 38, 39 and 40 are in a "normal" region, 41 and 42 in an "anomalous" one. Figures 38 and 39 show that the triangular shape is expanding, while Figure 40 shows the beginning of a contracting phase. As we enter the first "anomalous" region the area rapidly contracts, as seen in Figure 41, and after that rapidly expands, as shown in Figure 42. Now, as we reenter a "normal" region, the area keeps expanding (Figures 43 and 44) and then recontracts, as seen in Figures 45 and 46. This shape does not change much in the second "anomalous" region (Figure 47) or in the subsequent "normal" region (Figure 48).

To understand the behavior of tune versus emittance better, we modified the code PATRIS so that it can evaluate and print not only the emittance dependent tune, i.e. the position of the highest peak in the betatron oscillation frequency spectrum, but also the magnitudes and positions of the first ten most pronounced peaks. The results of this analysis for the first three peaks are shown in Tables 6,7 and 8. Apparently, there are two strongest peaks, one typically in the neighborhood of 0.825 and the other around 0.650 . The secondary peak obviously grows larger in these "anomalous" regions and surpasses the main peak - interpreted as the tune at that emittance. Also, our analysis shows that as we increase $\epsilon_{\mathrm{y}}$ the normal and anomalous regions alternate from $\epsilon_{\mathrm{y}}=0$ until $\epsilon_{\mathrm{y}}$ for which the particle reaches the edge of the dynamic aperture and gets lost. The reader will notice from Table 6 that we started with $0.00000001 \pi \mathrm{mm} \cdot \mathrm{mrad}$ as the smallest emittance. We had to move slightly away from the exact zero to get any betatron motion. The horizontal and vertical tune are respectively 0.825 and 0.824 , which matches the analytic values obtained from the lattice transfer matrix, to the accuracy of 0.001 which is dictated by $n=1000$ turns used in our tracking. We also notice the presence of very small peaks 
in the columns marked by PEAKX and PEAKY, respectively. In Table 7, which covers the section describing the second peak, we notice that the value DNUEX2, describing the position of the second peak, is 0.649 , which means that the second peak for $X$ direction was developed, even though it was too small to be printed as a non-zero value in the column marked by PEAKX2. However, DNUEY2 is 1.0 which means that the second peak did not develop for $Y$ direction at this small value of the emittance. We also notice that the presence of 1.0 in the columns DNUEX3 and DNUEY3 of Table 8 means that the third peak did not develop for both $X$ and $Y$ direction. However, as soon as we move away from this tiny emittance all three peaks, as well as the higher ones, develop.

Tables 6, 7 and 8 explain the origin of these "anomalous" regions. In the vertical plane we notice a very strong highest peak and heavily suppressed second and third peak, which are of the same magnitude. Therefore, the highest peak stays at its position 0.824 or slowly moves away in a monotonic dependence on the increased emittance. This position is then interpreted as tune at that emittance. In the horizontal plane the situation is very different. The motion is excited by the coupling and is small compared to the vertical motion. The first two highest peaks are of the same order of magnitude, while the third one is suppressed when compared with the first two, especially for not too large emittances. In those "anomalous" regions the second peak, positioned around 0.650 , surpasses in magnitude the peak positioned around 0.825 . We also observed that locations of these "anomalous" regions depend on the observation point. Some confusion was initially created after we moved the observation point from the beginning of the lattice to the last sextupole before the insertion. We observed the interval from 4.0 to $4.6 \pi \mathrm{mm} \cdot \mathrm{mrad}$, i.e. from 4.0 to the edge of the physical aperture and noticed that the "anomalies" suddenly disappeared. Only after extending exploration throughout the whole physical aperture, we "rediscovered anomalies," but now at different locations in the emittance interval. The reasons for this strange behavior are now obvious. As Figures 38 through 48 indicate, the $X$ motion of the particles, within the triangular shape, shows elements of chaoticity, and with the first and second peak being of the same magnitude it is obvious that the local beta function will have some influence in deciding which of the two peaks will prevail at some spot in the lattice. Also, our analysis shows that as we increase $\epsilon_{\mathrm{y}}$ the "normal" 
and "anomalous" regions alternate from $\epsilon_{\mathrm{y}}=0$ until $\epsilon_{\mathrm{y}}$ for which the particle reaches the edge of the dynamic aperture and gets lost.

Now we are ready to display tune dependence on the emittance. The tune is obviously determined by the peak which starts from the position matching the tune value determined analytically from the lattice transfer matrix and which is in most cases, i.e. outside those anomalous dips, still the highest peak. As far as the tune value is concerned, these dips can simply be ignored and the tune value interpolated in these narrow regions. A dip (or bump) over a wider region would, of course, require looking at the printout of the second peak and reading the appropriate values for the tune in an "anomalous" region there. However, we did not face that problem at least not at several places in the lattice where we observed the tune dependence on the emittance. It is also possible that a finer subdivision of the emittance interval and a more dense probing would reveal the presence of other narrower dips (or maybe bumps), but we did not do that exercise since it would not alter the whole qualitative picture. The tune dependence is now given in Figure 49. The dips can simply be ignored. In the figure they appear as missing points (i.e. as missing hollow circles) since the scale used for plotting does not allow them to appear within the page. We show for comparison also the tune variations evaluated by MAD and SYNCH. The agreement is good, especially in the vertical plane where the motion is essentially linear in $Y$, with $X$ acting as a small perturbation of the focusing strength constant $K$, as seen from Eq. (5).

Given the fact that we discovered a third-integer resonance, we wanted to see if it played any direct role in the particle loss. To determine this, we first moved the observation point from the initial place from which we launched the particles to the lattice bottleneck BC2. We generated phase space diagrams in the variables $\left(X, X^{\prime}, Y, Y^{\prime}\right)$ and in addition to small triangular shapes in the $\left(X, X^{\prime}\right)$ variables we also observed tilted and elongated ellipses in the $\left(Y, Y^{\prime}\right)$ plane, which indicated that particles were lost as a result of hitting the vacuum chamber, essentially on its top or bottom ( $X$ remains small when compared with $Y$ ).

We then removed physical aperture restrictions and examined the behavior of the particles until they reach the edge of the dynamic aperture and beyond. We generated phase space diagrams in Floquet variables at the lattice bottleneck BC2. An example of a particle well within the dynamic aperture is shown in Figures 50 and 51. The horizontal motion. 
remains confined. Note that Figure 50, describing the horizontal motion, is magnified by a factor 5 when compared with Figure 51. Figures 52 and 53 display the situation near the edge of the dynamic aperture. Notice the fairly rounded corners of the triangular shape in Figure 52. In Figures 54 and 55 we display the horizontal phase space diagrams for a particle slightly beyond the edge of the dynamic aperture. Figure 55 presents the same situation as Figure 54, but its magnification factor was taken to be only 1.5 and not 5.0 to prevent some particles from being outside the diagram. Figure 56 shows the vertical motion for the same particle as in Figures 54 and 55. From Figures 51, 53 and 56, we see that the ellipses describing the vertical motion steadily expand until the stability of the particle motion is lost. On the other hand, the triangular, densely filled shape, describing the horizontal motion, does not display any tendency to expand initially.

\section{Conclusion}

A careful analysis of tracking results with different initial conditions and small coordinate increments has revealed to us a behavior of motion with large variations. We have offered physical explanations which at closer look we judge reasonable and justified. Because of these results, we like to caution against tendencies to determine physical/dynamic aperture in storage rings by simply adopting the $X=Y$ case and proceeding from larger to lower amplitudes of motion. Actually, because of the fragmented behavior in some of the explored directions, we have some concerns about the definition itself of the "aperture".

\section{References}

1. A.G. Ruggiero, "RHICAGR. A most simplified RHIC lattice", AD/AP-26, BNL, August 1991.

2. J. Milutinovic and A. G. Ruggiero, "Long-term Tracking with PATRIS. Part 1. Search of Physical and Dynamic Aperture with Sextupoles", AD/AP-34, BNL, January 1992.

3. J. Milutinovic and A.G. Ruggiero, "Comparison of Accelerator Codes for a RHIC Lattice", AD/AD/TN-9, BNL, 1988.

4. H. Grote and F.C. Iselin, "The MAD Program, Ver. 8.4", CERN/SL/90-13 (AP) (Rev.2). 
5. A.A. Garren, et al., "A User's Guide to SYNCH", FN-420, Fermilab, June 1985.

6. T.L. Collins, "Distortion Functions", Fermilab-84/114, Fermilab, October 1984. 
Table 1. On-Momentum Physical/Dynamic Aperture without or with the Sextupoles

Physical Aperture - Sextupoles Off

\begin{tabular}{ccc} 
Dir. & Epsx & \multicolumn{2}{c}{ Epsy } \\
1. & {$\left[\begin{array}{cc}5.08, & 5.08\end{array}\right]$} & {$\left[\begin{array}{ll}0.00, & 0.00\end{array}\right]$} \\
2. & {$\left[\begin{array}{ll}2.54, & 2.59\end{array}\right]$} & {$\left[\begin{array}{ll}2.54, & 2.59\end{array}\right]$} \\
3. & {$\left[\begin{array}{ll}0.00, & 0.00\end{array}\right]$} & {$\left[\begin{array}{ll}5.08, & 5.08\end{array}\right]$} \\
4. & {$\left[\begin{array}{ll}2.54, & 2.59\end{array}\right]$} & {$\left[\begin{array}{ll}2.54, & 2.59\end{array}\right]$} \\
5. & {$\left[\begin{array}{ll}5.08, & 5.08\end{array}\right]$} & {$\left[\begin{array}{ll}0.00, & 0.00\end{array}\right]$} \\
6. & {$\left[\begin{array}{ll}2.54, & 2.59\end{array}\right]$} & {$\left[\begin{array}{ll}2.54, & 2.59\end{array}\right]$} \\
7. & {$\left[\begin{array}{ll}0.00, & 0.00\end{array}\right]$} & {$\left[\begin{array}{ll}5.08, & 5.08\end{array}\right]$} \\
8. & {$\left[\begin{array}{ll}2.54, & 2.59\end{array}\right]$} & {$\left[\begin{array}{ll}2.54, & 2.59\end{array}\right]$}
\end{tabular}

Physical Aperture - Sextupoles On

Dir.

Epsx

Epsy

\begin{tabular}{|c|c|c|c|}
\hline $\begin{array}{l}1 . \\
2 . \\
3 . \\
4 . \\
5 . \\
6 . \\
7 .\end{array}$ & 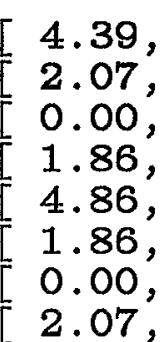 & $\begin{array}{l}4.39] \\
2.08] \\
0.00] \\
1.86] \\
4.87] \\
1.86] \\
0.00] \\
2.08]\end{array}$ & {$\left[\begin{array}{l}0.00, \\
2.07, \\
4.61, \\
1.86, \\
0.00, \\
1.86, \\
4.61, \\
2.07,\end{array}\right.$} \\
\hline
\end{tabular}

Dynamic Aperture - Sextupoles On

Dir.

Epsx

Epsy

\begin{tabular}{|c|c|c|c|}
\hline $\begin{array}{l}1 . \\
2 . \\
3 . \\
4 . \\
5 . \\
6 .\end{array}$ & 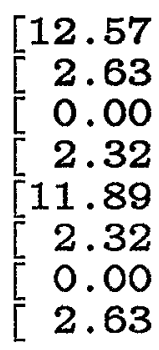 & $\begin{array}{r}12.57] \\
2.63] \\
0.00] \\
2.32] \\
11.89] \\
2.32] \\
0.00] \\
2.63]\end{array}$ & 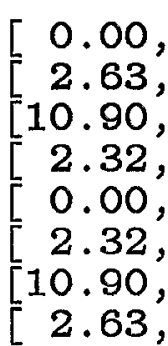 \\
\hline
\end{tabular}


Table 2. Initial Emittances and Surviving Number of Turns for a Set of Particles Launched in Direction \# 4

$\begin{array}{rrc}\text { PART.\# } & \text { TURNS } & \text { EPSX=EPSY } \\ 1 & 1000 & \\ 1 & 731 & 2.822 \\ 2 & 96 & 2.973 \\ 3 & 3.124 \\ 4 & 151 & 3.275 \\ 5 & 1000 & 3.426 \\ 6 & 198 & 3.578 \\ 7 & 1000 & 3.729 \\ 8 & 1000 & 3.880 \\ 9 & 1000 & 4.031 \\ 10 & 1000 & 4.182 \\ 11 & 1000 & 4.333 \\ 12 & 1000 & 4.484 \\ 13 & 1000 & 4.635 \\ 14 & 1000 & 4.787 \\ 15 & 1000 & 4.938 \\ 16 & 1000 & 5.089 \\ 17 & 1000 & 5.240 \\ 18 & 722 & 5.391 \\ 19 & 1000 & 5.542 \\ 20 & 1000 & 5.693 \\ 21 & 474 & 5.844 \\ 22 & 1000 & 5.996 \\ 23 & 1000 & 6.147 \\ 24 & 1000 & 6.298 \\ 25 & 143 & 6.449 \\ 26 & 919 & 6.600\end{array}$


Table 3. Initial Emittances and Surviving Number of Turns for a Set of Particles Launched in Direction \#4 Interval between Part. \# 7 and \# 17 of Table 2 (First Subdivision)

$\begin{array}{crc}\text { PART.\# } & \text { TURNS } & \text { EPSX=EPSY } \\ 1 & 1000 & 3.729 \\ 2 & 1000 & 3.789 \\ 3 & 1000 & 3.850 \\ 4 & 1000 & 3.910 \\ 5 & 1000 & 3.971 \\ 6 & 1000 & 4.031 \\ 7 & 984 & 4.092 \\ 8 & 1000 & 4.152 \\ 9 & 1000 & 4.213 \\ 10 & 113 & 4.273 \\ 11 & 937 & 4.333 \\ 12 & 1000 & 4.394 \\ 13 & 1000 & 4.454 \\ 14 & 1000 & 4.515 \\ 15 & 1000 & 4.575 \\ 16 & 1000 & 4.636 \\ 17 & 1000 & 4.696 \\ 18 & 1000 & 4.756 \\ 19 & 1000 & 4.817 \\ 20 & 1000 & 4.877 \\ 21 & 1000 & 4.938 \\ 22 & 1000 & 4.998 \\ 23 & 1000 & 5.059 \\ 24 & 883 & 5.119 \\ 25 & 519 & 5.180 \\ 26 & 745 & 5.240\end{array}$


Table 4. Initial Emittances and Surviving Number of Turns for a Set of Particles Launched in Direction \# 4 Interval between Part. \# 7 and \# 17 of Table 2 (Second Subdivision)

$\begin{array}{rrc}\text { PART } \# & \text { TURNS } & \text { EPSX=EPSY } \\ 1 & 1000 & 3.729 \\ 2 & 1000 & 3.789 \\ 3 & 1000 & 3.850 \\ 4 & 1000 & 3.910 \\ 5 & 1000 & 3.971 \\ 6 & 1000 & 4.031 \\ 7 & 1000 & 4.091 \\ 8 & 742 & 4.152 \\ 9 & 1000 & 4.212 \\ 10 & 122 & 4.273 \\ 11 & 1000 & 4.333 \\ 12 & 691 & 4.393 \\ 13 & 1000 & 4.454 \\ 14 & 1000 & 4.514 \\ 15 & 1000 & 4.575 \\ 16 & 1000 & 4.635 \\ 17 & 162 & 4.695 \\ 18 & 978 & 4.756 \\ 19 & 379 & 4.816 \\ 20 & 510 & 4.877 \\ 21 & 1000 & 4.937 \\ 22 & 475 & 4.997 \\ 23 & 1000 & 5.058 \\ 24 & 1000 & 5.118 \\ 25 & 1000 & 5.179 \\ 26 & 1000 & 5.239\end{array}$


Table 5. Tune Dependence on the Amplitude - Direction \# 3

$\begin{array}{rcccc}\text { Part. \# } & \text { EPSX } & \text { DNUEX } & \text { EPSY } & \text { DNUEY } \\ 1 & 0.00000000 & 0.82500000 & 0.00000001 & 0.82400000 \\ 2 & 0.00000000 & 0.82500000 & 0.04000001 & 0.82400000 \\ 3 & 0.00000000 & 0.82500000 & 0.08000001 & 0.82400000 \\ 4 & 0.00000000 & 0.82500000 & 0.12000001 & 0.82400000 \\ 5 & 0.00000000 & 0.82500000 & 0.16000001 & 0.82500000 \\ 6 & 0.00000000 & 0.82500000 & 0.20000001 & 0.82500000 \\ 7 & 0.00000000 & 0.82500000 & 0.24000001 & 0.82500000 \\ 8 & 0.00000000 & 0.82500000 & 0.28000001 & 0.82500000 \\ 9 & 0.00000000 & 0.82500000 & 0.32000001 & 0.82500000 \\ 10 & 0.00000000 & 0.65000000 & 0.36000001 & 0.82500000 \\ 11 & 0.00000000 & 0.65000000 & 0.40000001 & 0.82500000 \\ 12 & 0.00000000 & 0.65000000 & 0.44000001 & 0.82500000 \\ 13 & 0.00000000 & 0.82500000 & 0.48000001 & 0.82500000 \\ 14 & 0.00000000 & 0.82500000 & 0.52000000 & 0.82500000 \\ 15 & 0.00000000 & 0.82500000 & 0.56000000 & 0.82500000 \\ 16 & 0.00000000 & 0.82500000 & 0.60000000 & 0.82500000 \\ 17 & 0.00000000 & 0.82500000 & 0.64000000 & 0.82500000 \\ 18 & 0.00000000 & 0.82500000 & 0.68000000 & 0.82500000 \\ 19 & 0.00000000 & 0.82400000 & 0.72000000 & 0.82500000 \\ 20 & 0.00000000 & 0.82400000 & 0.76000000 & 0.82500000 \\ 21 & 0.00000000 & 0.65100000 & 0.80000000 & 0.82500000 \\ 22 & 0.00000000 & 0.65100000 & 0.84000000 & 0.82500000 \\ 23 & 0.00000000 & 0.65100000 & 0.88000000 & 0.82500000 \\ 24 & 0.00000000 & 0.82400000 & 0.92000000 & 0.82600000 \\ 25 & 0.00000000 & 0.82400000 & 0.96000000 & 0.82600000 \\ 26 & 0.00000000 & 0.82400000 & 1.00000000 & 0.82600000\end{array}$


Table 6. First Peak in the Betatron Oscillation Frequency Spectrum Tune Dependence on the Amplitude

\begin{tabular}{|c|c|c|c|c|c|c|}
\hline Part. \# & EPSX & DNUEX & PEAKX & EPSY & DNUEY & PEAKY \\
\hline $\begin{array}{r}1 \\
2 \\
3 \\
4 \\
5 \\
6 \\
7 \\
8 \\
9 \\
10 \\
11 \\
12 \\
13 \\
14 \\
15 \\
16 \\
17 \\
18 \\
19 \\
20 \\
21 \\
22 \\
23 \\
24 \\
25 \\
26\end{array}$ & $\begin{array}{l}0.00000000 \\
0.00000000 \\
0.00000000 \\
0.00000000 \\
0.00000000 \\
0.00000000 \\
0.00000000 \\
0.00000000 \\
0.00000000 \\
0.00000000 \\
0.00000000 \\
0.00000000 \\
0.00000000 \\
0.00000000 \\
0.00000000 \\
0.00000000 \\
0.00000000 \\
0.00000000 \\
0.00000000 \\
0.00000000 \\
0.00000000 \\
0.00000000 \\
0.00000000 \\
0.00000000 \\
0.00000000 \\
0.00000000\end{array}$ & $\begin{array}{l}0.82500000 \\
0.82500000 \\
0.82500000 \\
0.82500000 \\
0.82500000 \\
0.82500000 \\
0.82500000 \\
0.82500000 \\
0.82500000 \\
0.65000000 \\
0.65000000 \\
0.65000000 \\
0.82500000 \\
0.82500000 \\
0.82500000 \\
0.82500000 \\
0.82500000 \\
0.82500000 \\
0.82400000 \\
0.82400000 \\
0.65100000 \\
0.65100000 \\
0.65100000 \\
0.82400000 \\
0.82400000 \\
0.82400000\end{array}$ & $\begin{array}{r}0.000001 \\
2.707336 \\
6.195633 \\
11.271441 \\
17.905918 \\
24.867461 \\
30.077143 \\
31.227762 \\
26.478159 \\
15.591276 \\
19.408744 \\
22.882714 \\
40.879733 \\
55.026320 \\
61.733978 \\
60.174921 \\
51.648002 \\
39.756822 \\
32.246198 \\
36.885005 \\
40.112398 \\
44.585529 \\
47.700273 \\
52.584487 \\
56.901329 \\
58.844836\end{array}$ & $\begin{array}{l}0.00000001 \\
0.04000001 \\
0.08000001 \\
0.12000001 \\
0.16000001 \\
0.20000001 \\
0.24000001 \\
0.28000001 \\
0.32000001 \\
0.36000001 \\
0.40000001 \\
0.44000001 \\
0.48000001 \\
0.52000000 \\
0.56000000 \\
0.60000000 \\
0.64000000 \\
0.68000000 \\
0.72000000 \\
0.76000000 \\
0.80000000 \\
0.84000000 \\
0.88000000 \\
0.92000000 \\
0.96000000 \\
1.00000000\end{array}$ & $\begin{array}{l}0.82400000 \\
0.82400000 \\
0.82400000 \\
0.82400000 \\
0.82500000 \\
0.82500000 \\
0.82500000 \\
0.82500000 \\
0.82500000 \\
0.82500000 \\
0.82500000 \\
0.82500000 \\
0.82500000 \\
0.82500000 \\
0.82500000 \\
0.82500000 \\
0.82500000 \\
0.82500000 \\
0.82500000 \\
0.82500000 \\
0.82500000 \\
0.82500000 \\
0.82500000 \\
0.82600000 \\
0.82600000 \\
0.82600000\end{array}$ & $\begin{array}{r}0.293555 \\
546.885303 \\
710.683575 \\
787.904911 \\
987.837296 \\
1203.921873 \\
1418.238910 \\
1627.676769 \\
1828.317529 \\
2015.256243 \\
2183.377284 \\
2328.911600 \\
2450.316045 \\
2547.283229 \\
2618.851031 \\
2662.720486 \\
2676.372346 \\
2658.643713 \\
2610.170551 \\
2532.480955 \\
2427.067551 \\
2295.527669 \\
2140.205621 \\
2362.991826 \\
2620.410214 \\
2867.785943\end{array}$ \\
\hline
\end{tabular}


Table 7. Second Peak in the Betatron Oscillation Frequency Spectrum

\begin{tabular}{|c|c|c|c|c|c|c|}
\hline Part. \# & EPSX & DNUEX2 & PEAKX2 & EPSY & DNUEY 2 & PEAKY2 \\
\hline $\begin{array}{r}1 \\
2 \\
3 \\
4 \\
5 \\
6 \\
7 \\
8 \\
9 \\
10 \\
11 \\
12 \\
13 \\
14 \\
15 \\
16 \\
17 \\
18 \\
19 \\
20 \\
21 \\
22 \\
23 \\
24 \\
25 \\
26\end{array}$ & $\begin{array}{l}0.00000000 \\
0.00000000 \\
0.00000000 \\
0.00000000 \\
0.00000000 \\
0.00000000 \\
0.00000000 \\
0.00000000 \\
0.00000000 \\
0.00000000 \\
0.00000000 \\
0.00000000 \\
0.00000000 \\
0.00000000 \\
0.00000000 \\
0.00000000 \\
0.00000000 \\
0.00000000 \\
0.00000000 \\
0.00000000 \\
0.00000000 \\
0.00000000 \\
0.00000000 \\
0.00000000 \\
0.00000000 \\
0.00000000\end{array}$ & $\begin{array}{l}0.64900000 \\
0.64900000 \\
0.64900000 \\
0.64900000 \\
0.64900000 \\
0.64900000 \\
0.64900000 \\
0.64900000 \\
0.65000000 \\
0.82500000 \\
0.82400000 \\
0.82500000 \\
0.65000000 \\
0.65000000 \\
0.65000000 \\
0.65000000 \\
0.65000000 \\
0.65000000 \\
0.65100000 \\
0.65100000 \\
0.82400000 \\
0.82400000 \\
0.82400000 \\
0.65100000 \\
0.65100000 \\
0.65100000\end{array}$ & $\begin{array}{r}0.000000 \\
1.985193 \\
4.227346 \\
6.480984 \\
8.497409 \\
10.051886 \\
10.956820 \\
11.074539 \\
11.732394 \\
15.142403 \\
8.426323 \\
21.953666 \\
25.770509 \\
27.857628 \\
28.920790 \\
28.757540 \\
27.268477 \\
24.518730 \\
28.647198 \\
34.677966 \\
39.219719 \\
41.896415 \\
46.767104 \\
49.153031 \\
48.832867 \\
46.774423\end{array}$ & $\begin{array}{l}0.000000001 \\
0.04000001 \\
0.08000001 \\
0.12000001 \\
0.16000001 \\
0.20000001 \\
0.24000001 \\
0.28000001 \\
0.32000001 \\
0.36000001 \\
0.40000001 \\
0.44000001 \\
0.48000001 \\
0.52000000 \\
0.56000000 \\
0.60000000 \\
0.64000000 \\
0.68000000 \\
0.72000000 \\
0.76000000 \\
0.80000000 \\
0.84000000 \\
0.88000000 \\
0.92000000 \\
0.96000000 \\
1.00000000\end{array}$ & $\begin{array}{l}1.00000000 \\
0.65000000 \\
0.65000000 \\
0.65000000 \\
0.65000000 \\
0.65000000 \\
0.65000000 \\
0.65000000 \\
0.65000000 \\
0.65000000 \\
0.52500000 \\
0.52500000 \\
0.65000000 \\
0.65000000 \\
0.65000000 \\
0.65000000 \\
0.65000000 \\
0.70000000 \\
0.65100000 \\
0.70000000 \\
0.70000000 \\
0.69700000 \\
0.65000000 \\
0.65000000 \\
0.65000000 \\
0.65000000\end{array}$ & $\begin{array}{r}0.000000 \\
1.918656 \\
2.751119 \\
3.278641 \\
3.613623 \\
3.797896 \\
3.760866 \\
3.426772 \\
2.820279 \\
1.951717 \\
1.349582 \\
1.953904 \\
3.491997 \\
5.368517 \\
6.324987 \\
6.140064 \\
5.513586 \\
7.176460 \\
8.951187 \\
9.997859 \\
10.751294 \\
10.904698 \\
10.249691 \\
10.473670 \\
10.498926 \\
10.192871\end{array}$ \\
\hline
\end{tabular}


Table 8. Third Peak in the Betatron Oscillation Frequency Spectrum

\begin{tabular}{|c|c|c|c|c|c|c|}
\hline Part. \# & EPSX & DNUEX3 & PEAKX3 & EPSY & DNUEY 3 & PEAKY3 \\
\hline $\begin{array}{r}1 \\
2 \\
3 \\
4 \\
5 \\
6 \\
7 \\
8 \\
9 \\
10 \\
11 \\
12 \\
13 \\
14 \\
15 \\
16 \\
17 \\
18 \\
19 \\
20 \\
21 \\
22 \\
23 \\
24 \\
25 \\
26\end{array}$ & $\begin{array}{l}0.00000000 \\
0.00000000 \\
0.00000000 \\
0.00000000 \\
0.00000000 \\
0.00000000 \\
0.00000000 \\
0.00000000 \\
0.00000000 \\
0.00000000 \\
0.00000000 \\
0.00000000 \\
0.00000000 \\
0.00000000 \\
0.00000000 \\
0.00000000 \\
0.00000000 \\
0.00000000 \\
0.00000000 \\
0.00000000 \\
0.00000000 \\
0.00000000 \\
0.00000000 \\
0.00000000 \\
0.00000000 \\
0.00000000\end{array}$ & $\begin{array}{l}1.00000000 \\
0.52600000 \\
0.70200000 \\
0.70200000 \\
0.70100000 \\
0.70100000 \\
0.70100000 \\
0.70100000 \\
0.70000000 \\
0.70100000 \\
0.82600000 \\
0.70000000 \\
0.70000000 \\
0.70000000 \\
0.69900000 \\
0.69900000 \\
0.69900000 \\
0.69900000 \\
0.69900000 \\
0.82600000 \\
0.82600000 \\
0.69800000 \\
0.82700000 \\
0.82700000 \\
0.82700000 \\
0.82700000\end{array}$ & $\begin{array}{r}0.000000 \\
0.010761 \\
0.027963 \\
0.073681 \\
0.102901 \\
0.180663 \\
0.236774 \\
0.189214 \\
0.143691 \\
0.215942 \\
6.373534 \\
0.457387 \\
0.724482 \\
0.725364 \\
0.514806 \\
0.732018 \\
0.757789 \\
0.749951 \\
0.938930 \\
27.374020 \\
28.033887 \\
1.255497 \\
23.403429 \\
28.704833 \\
31.722357 \\
30.984136\end{array}$ & $\begin{array}{l}0.00000001 \\
0.04000001 \\
0.08000001 \\
0.12000001 \\
0.16000001 \\
0.20000001 \\
0.24000001 \\
0.28000001 \\
0.32000001 \\
0.36000001 \\
0.40000001 \\
0.44000001 \\
0.48000001 \\
0.52000000 \\
0.56000000 \\
0.60000000 \\
0.64000000 \\
0.68000000 \\
0.72000000 \\
0.76000000 \\
0.80000000 \\
0.84000000 \\
0.88000000 \\
0.92000000 \\
0.96000000 \\
1.00000000\end{array}$ & $\begin{array}{l}1.00000000 \\
0.64800000 \\
0.64600000 \\
0.64500000 \\
0.64300000 \\
0.64100000 \\
0.70100000 \\
0.70100000 \\
0.52600000 \\
0.52600000 \\
0.65100000 \\
0.65100000 \\
0.52500000 \\
0.52500000 \\
0.69900000 \\
0.69900000 \\
0.70000000 \\
0.69800000 \\
0.70000000 \\
0.65100000 \\
0.69700000 \\
0.65000000 \\
0.65200000 \\
0.69900000 \\
0.69900000 \\
0.69900000\end{array}$ & $\begin{array}{r}0.000000 \\
1.882478 \\
2.650209 \\
3.028792 \\
3.056876 \\
2.799274 \\
3.571815 \\
3.001524 \\
1.123687 \\
1.031045 \\
1.094120 \\
1.525651 \\
2.085287 \\
1.843270 \\
1.996966 \\
3.620966 \\
5.409419 \\
7.005587 \\
8.770877 \\
9.629756 \\
10.537692 \\
10.088618 \\
9.545553 \\
9.982693 \\
9.087993 \\
7.932669\end{array}$ \\
\hline
\end{tabular}




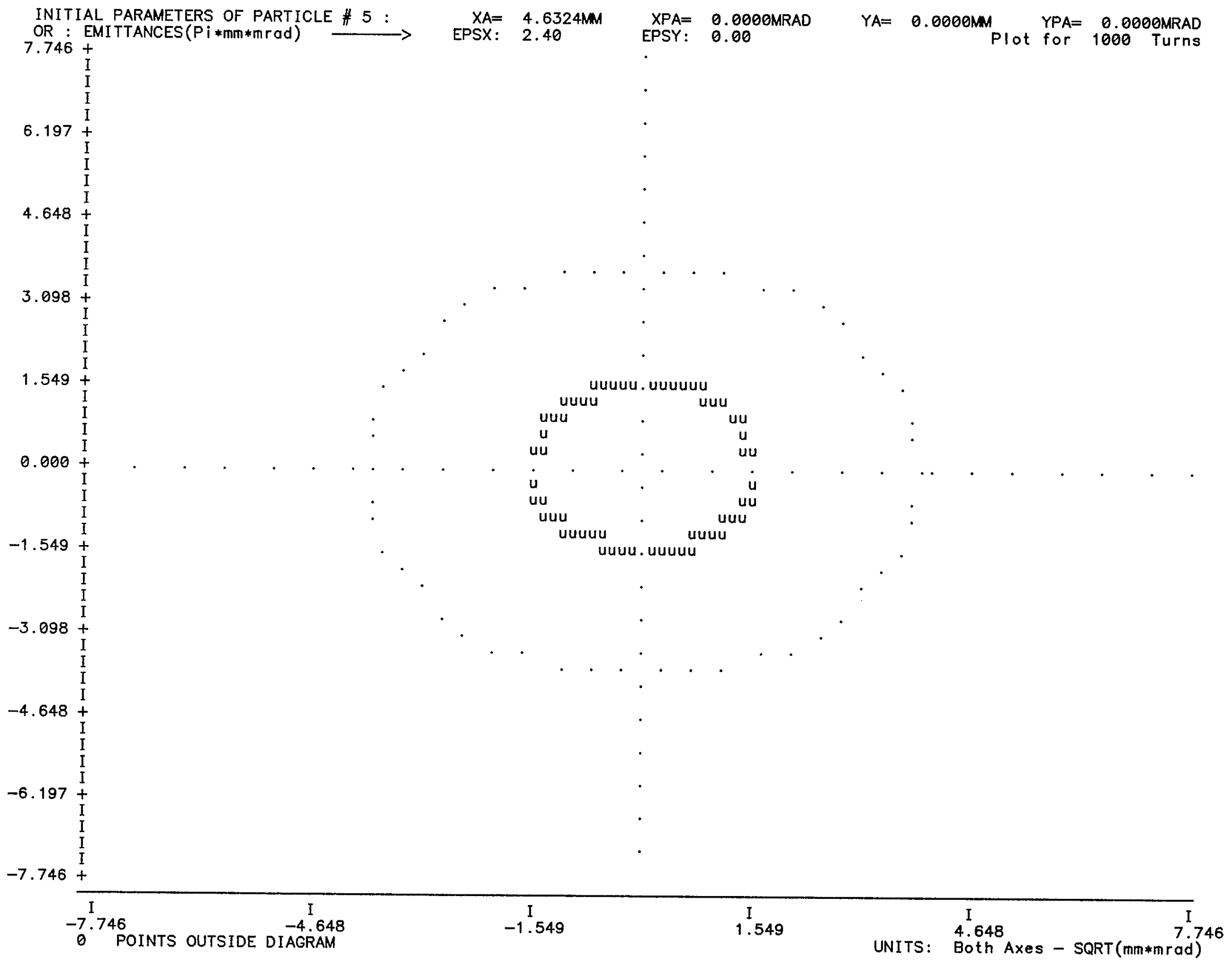

Figure 1. Particle trajectory in Floquet $\left(u, u^{\prime}\right)$ coordinates. Case \#1, small amplitude. 


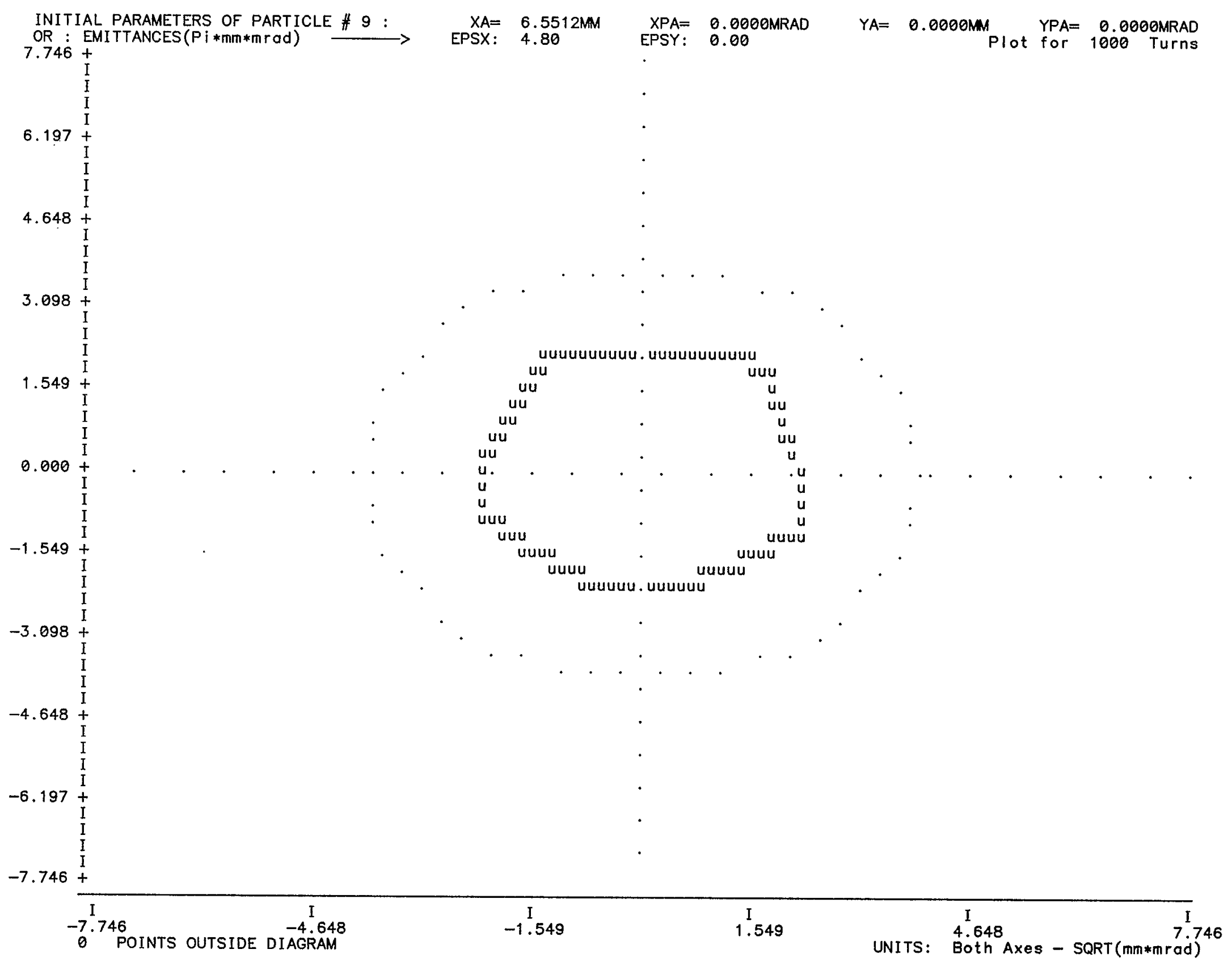

Figure 2. Particle trajectory in Floquet $\left(u, u^{\prime}\right)$ coordinates. Case \#1, medium amplitude. 


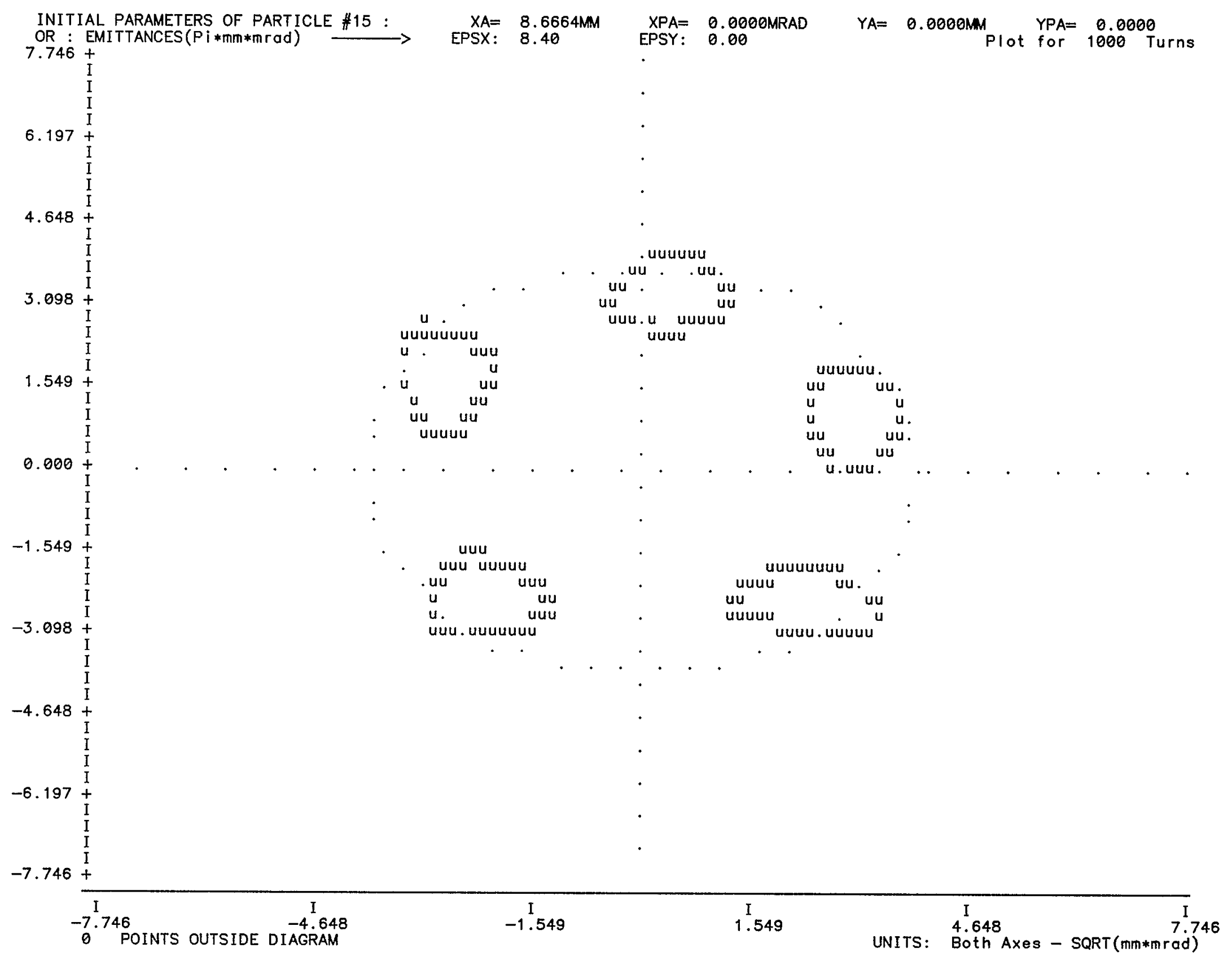

Figure 3. Particle trajectory in Floquet ( $\left.u, u^{\prime}\right)$ coordinates. Case \# 1, large amplitude. 


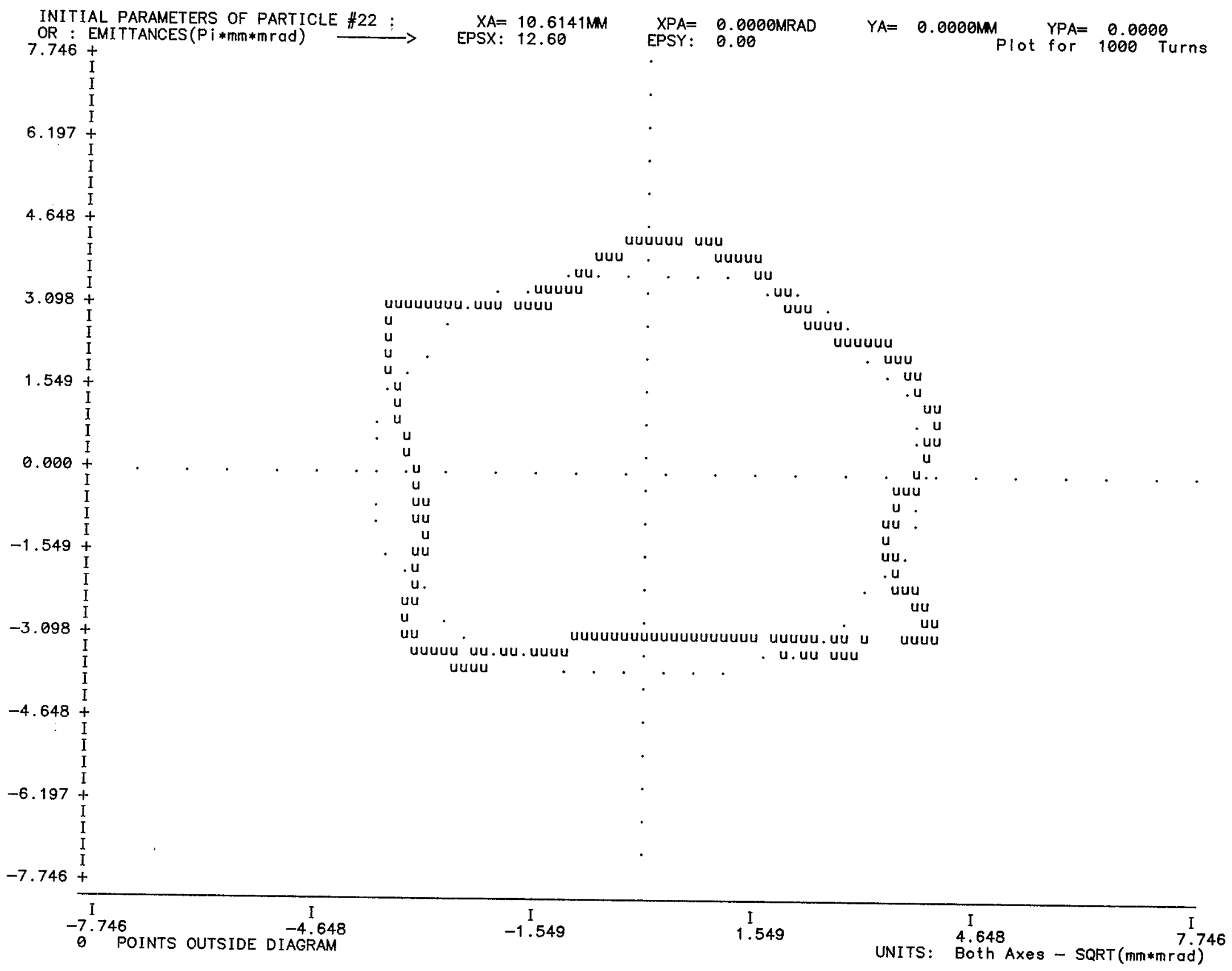

Figure 4. Particle trajectory in Floquet $\left(u, u^{\prime}\right)$ coordinates. Case \#1, amplitude close to dynamic aperture. 


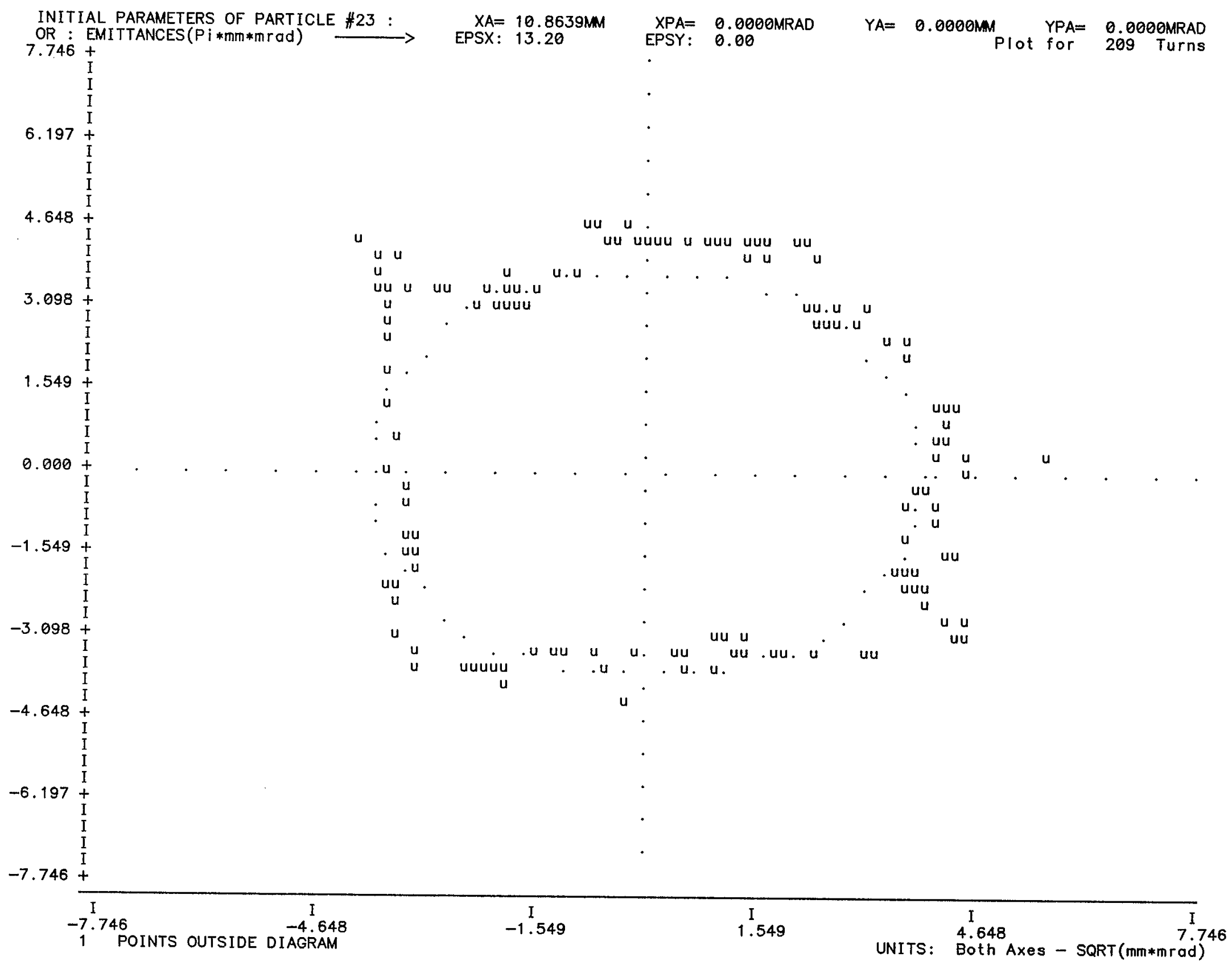

Figure 5. Particle trajectory in Floquet $\left(u, u^{\prime}\right)$ coordinates. Case \#1, amplitude just outside dynamic aperture. 


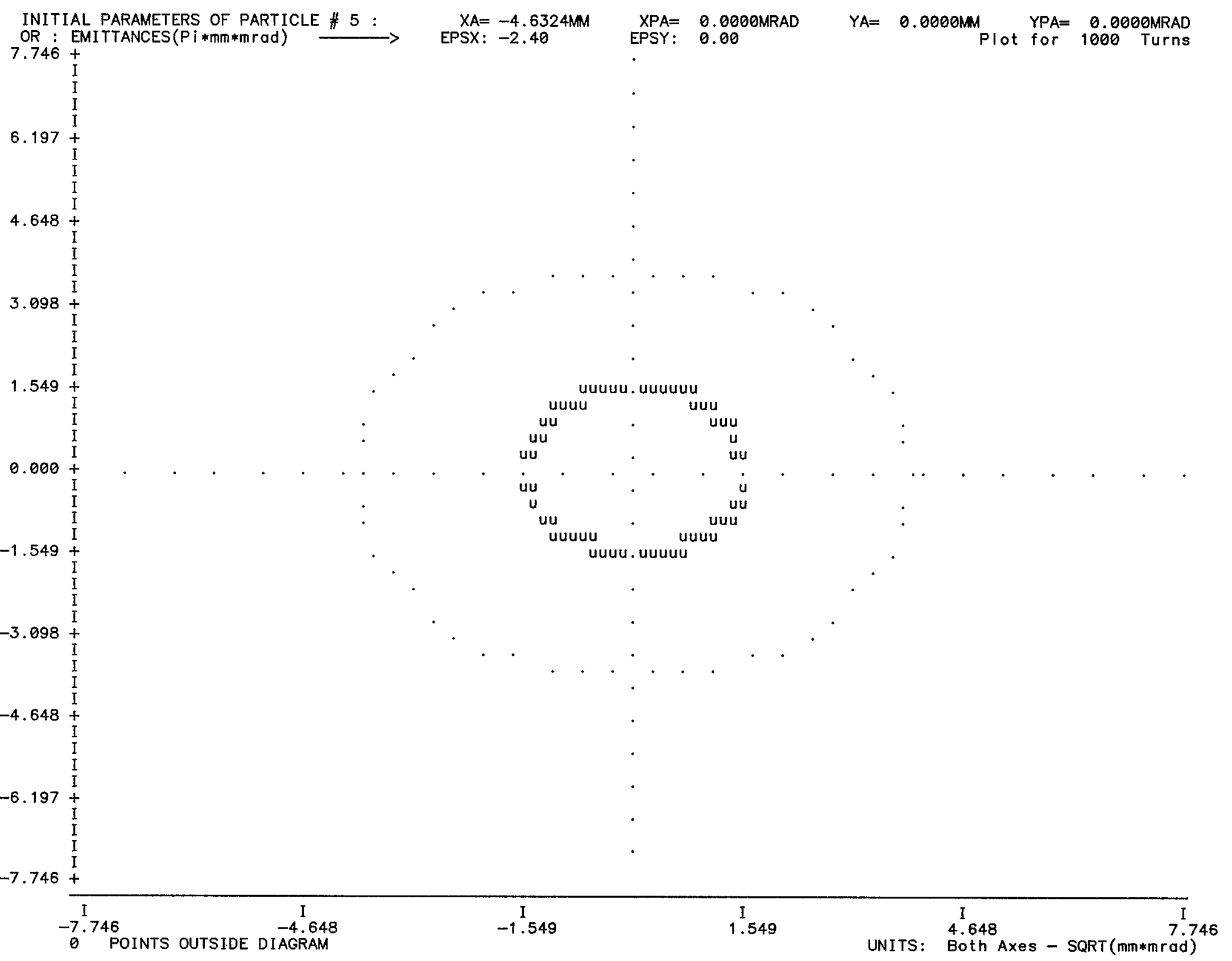

Figure 6. Particle trajectory in Floquet $\left(u, u^{\prime}\right)$ coordinates. Case \#5, small amplitude. 


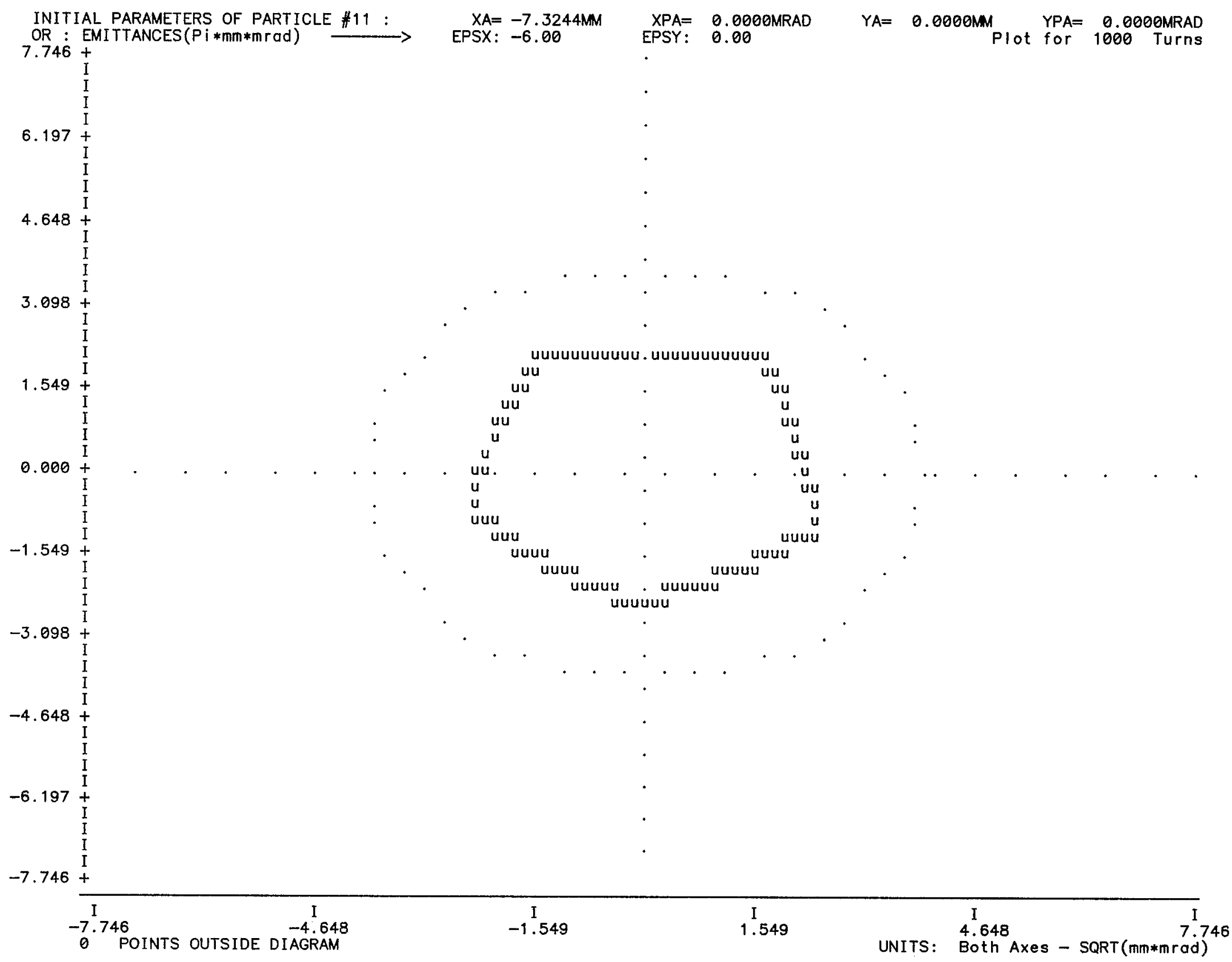

Figure 7. Particle trajectory in Floquet ( $u, u^{\prime}$ ) coordinates. Case \#5, medium amplitude. 


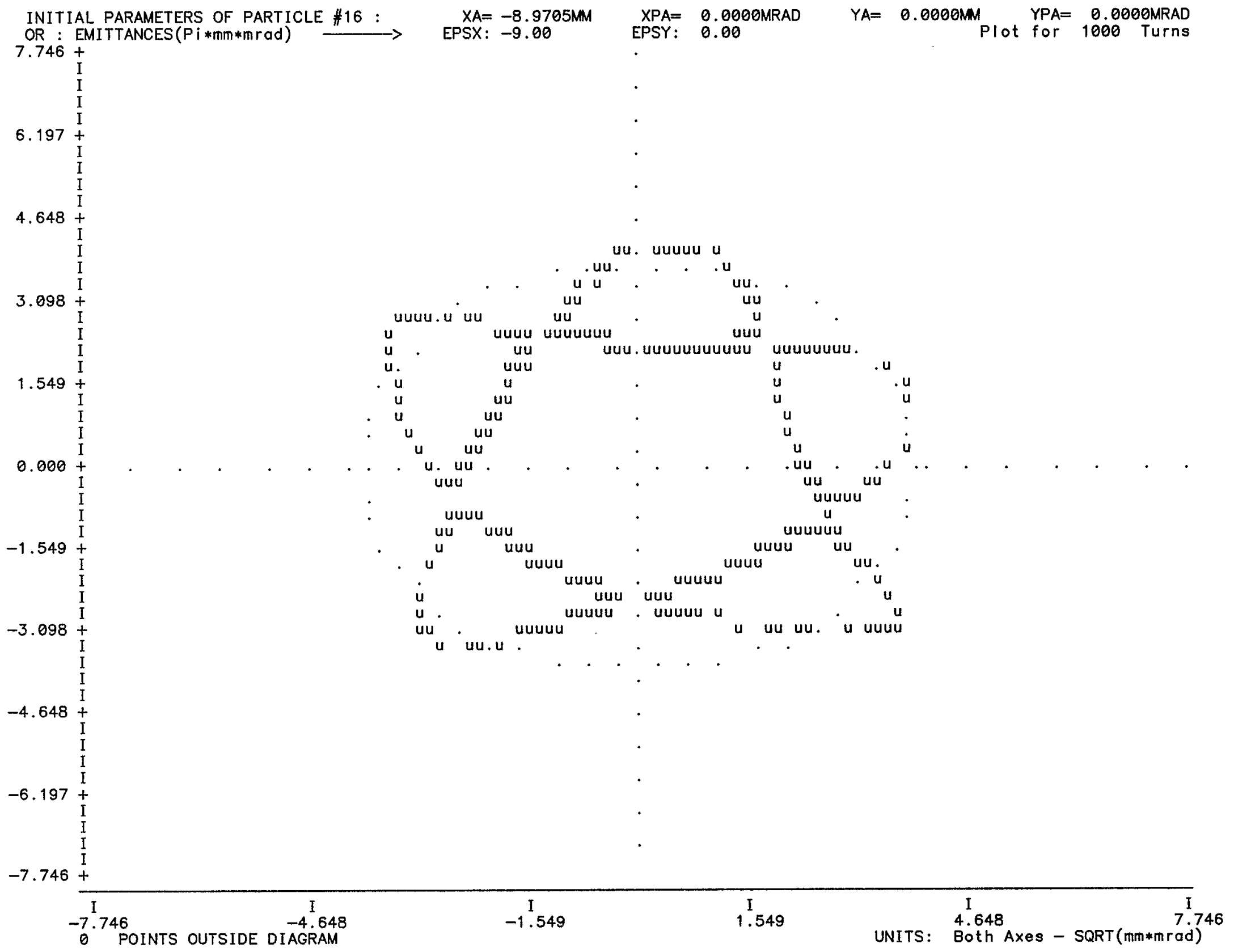

Figure 8. Particle trajectory in Floquet ( $\left.u, u^{\prime}\right)$ coordinates. Case \#5, large amplitude. 


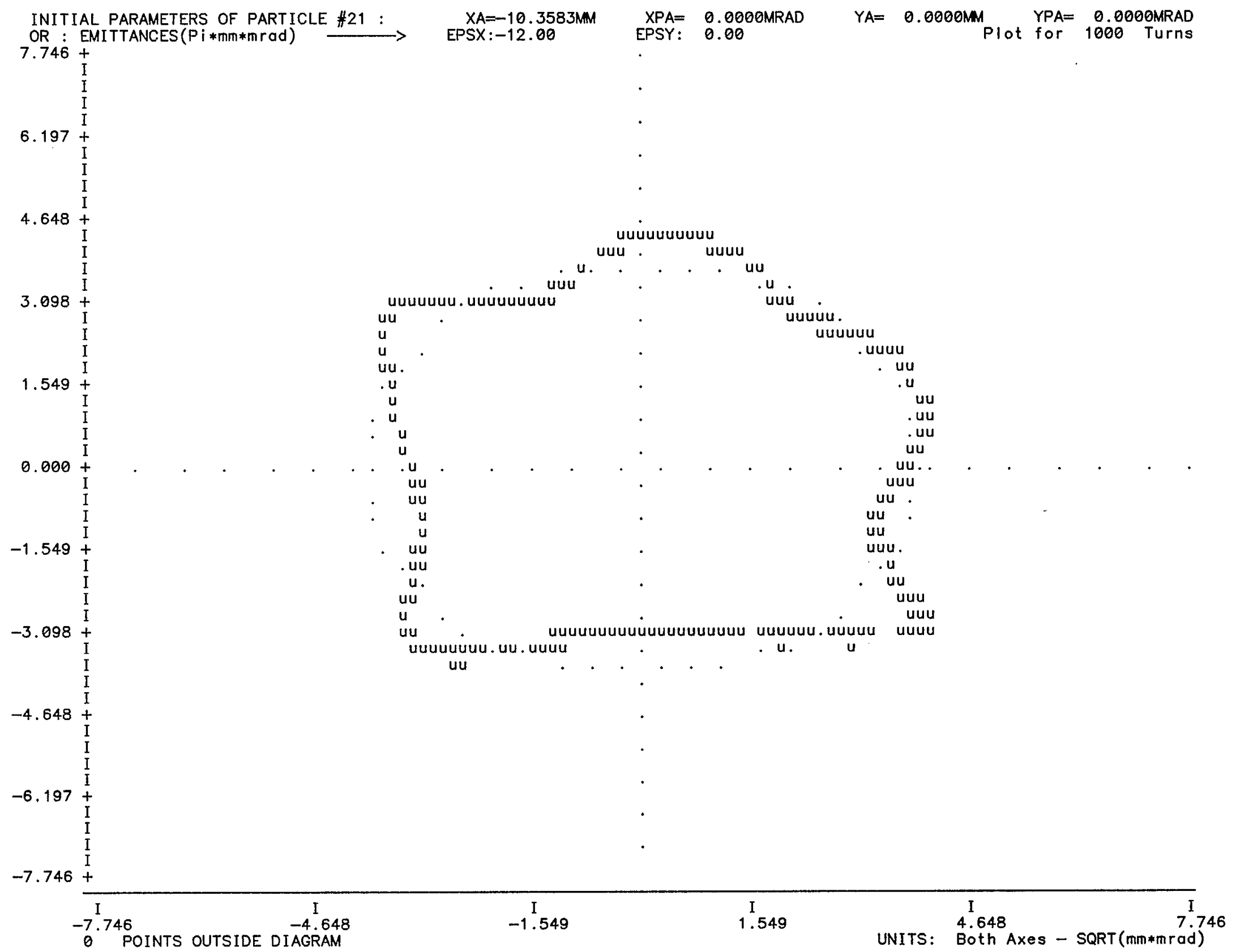

Figure 9. Particle trajectory in Floquet $\left(u, u^{\prime}\right)$ coordinates. Case \#5, amplitude close to dynamic aperture. 


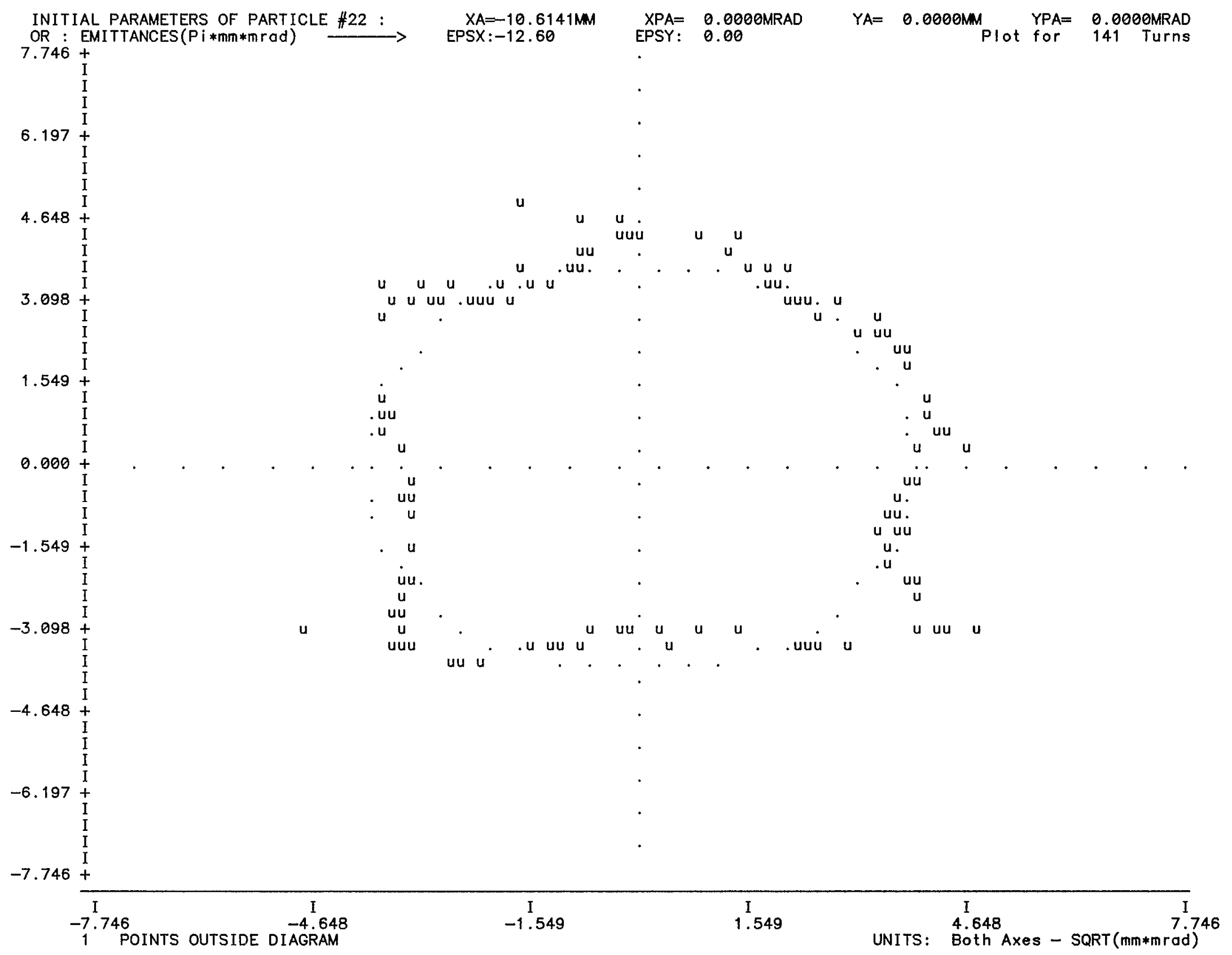

Figure 10. Particle trajectory in Floquet ( $u, u^{\prime}$ ) coordinates. Case \#5, amplitude just outside dynamic aperture. 


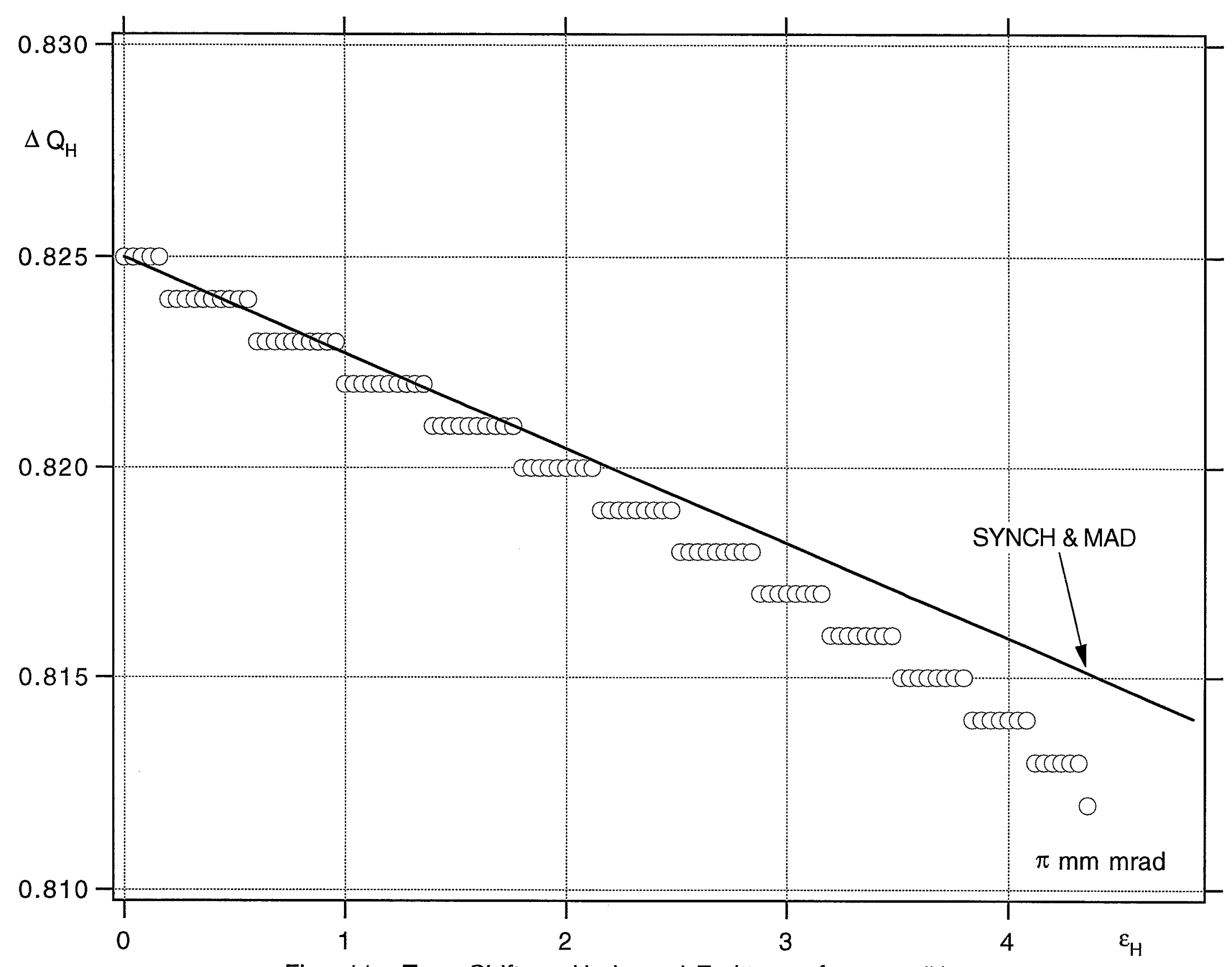

Fig. 11 Tune-Shift vs. Horizontal Emittance for case \#1 


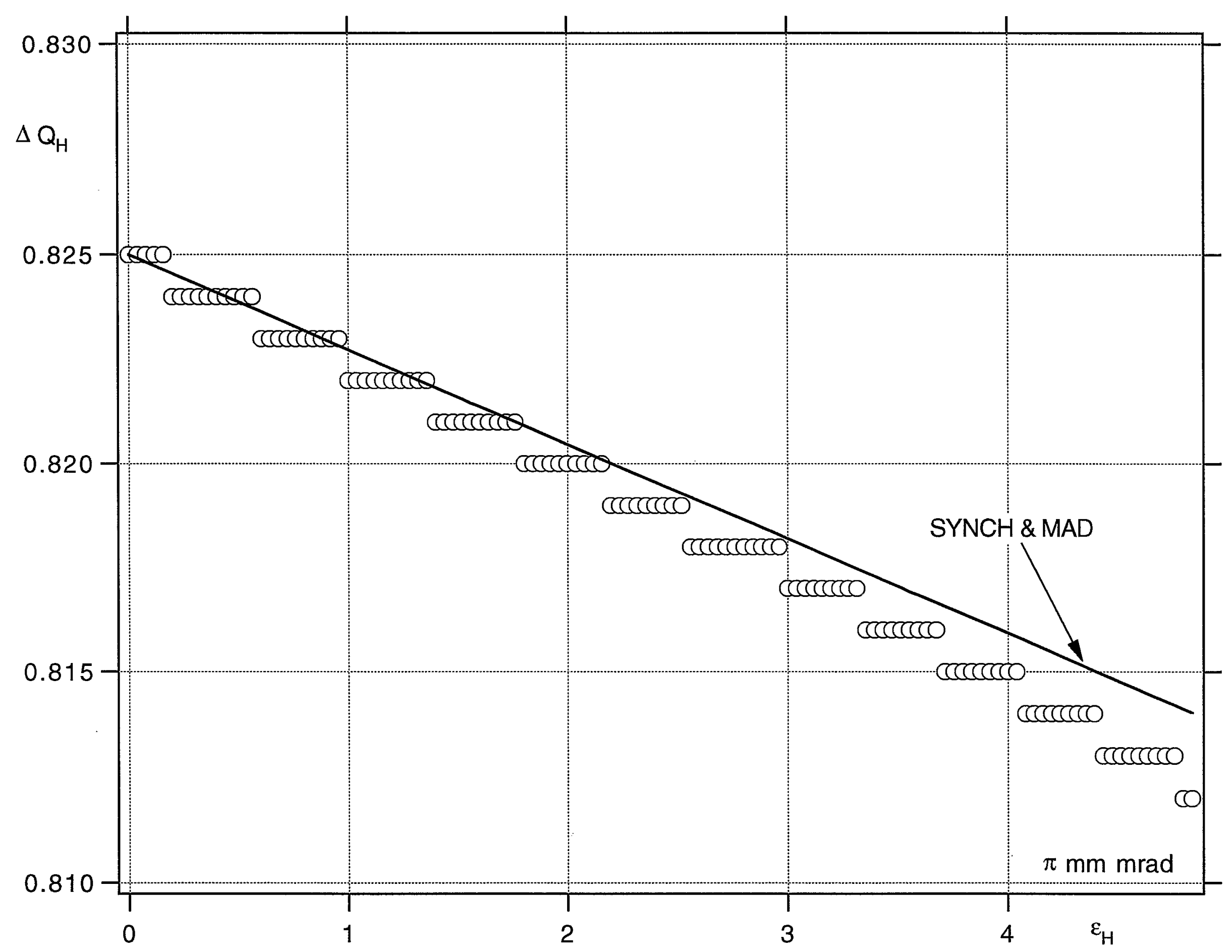

Fig. 12 Tune-Shift vs. Horizontal Emittance for case \#5 


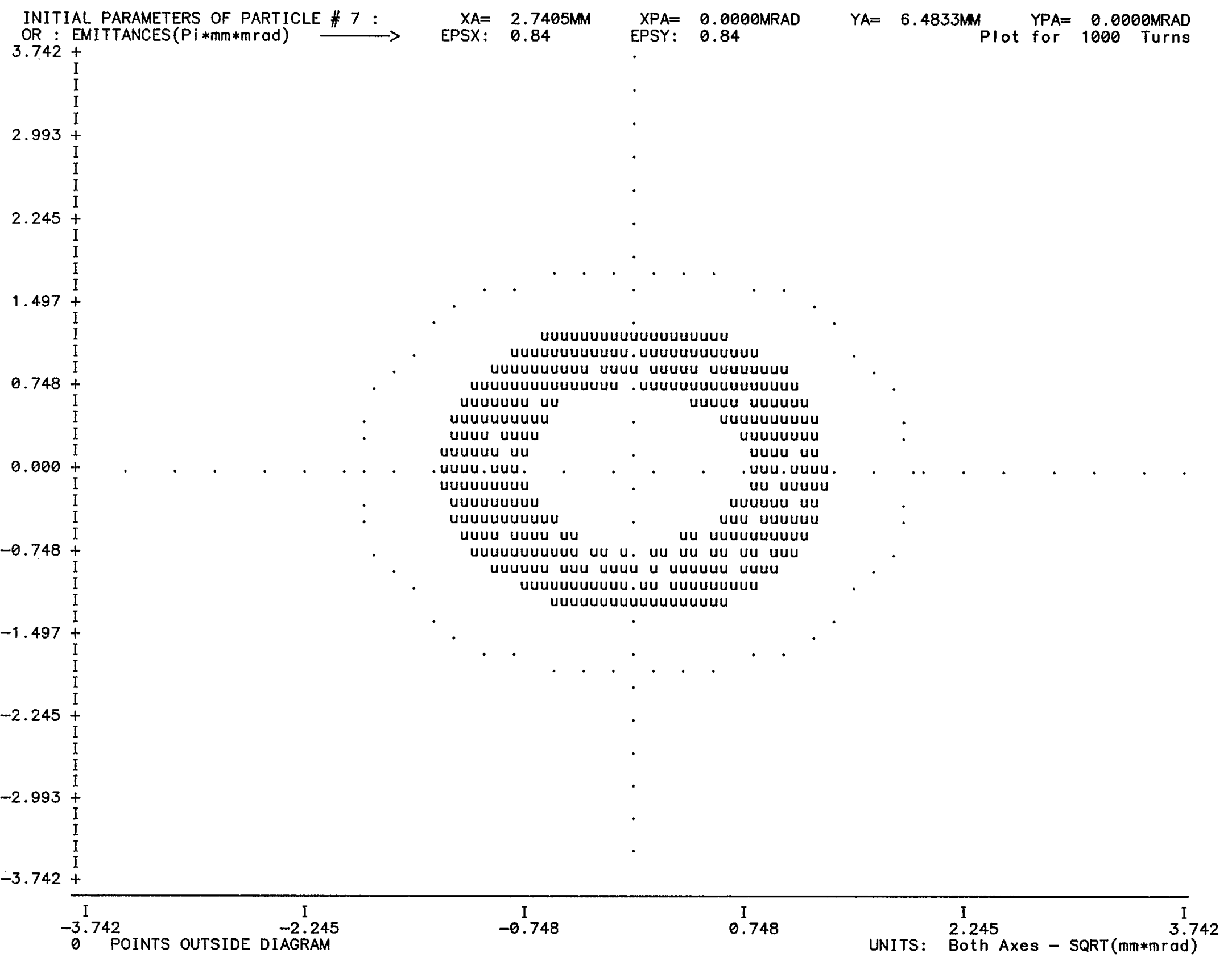

Figure 13. Particle trajectory in Floquet $\left(u, u^{\prime}\right)$ coordinates. Case \#2. 


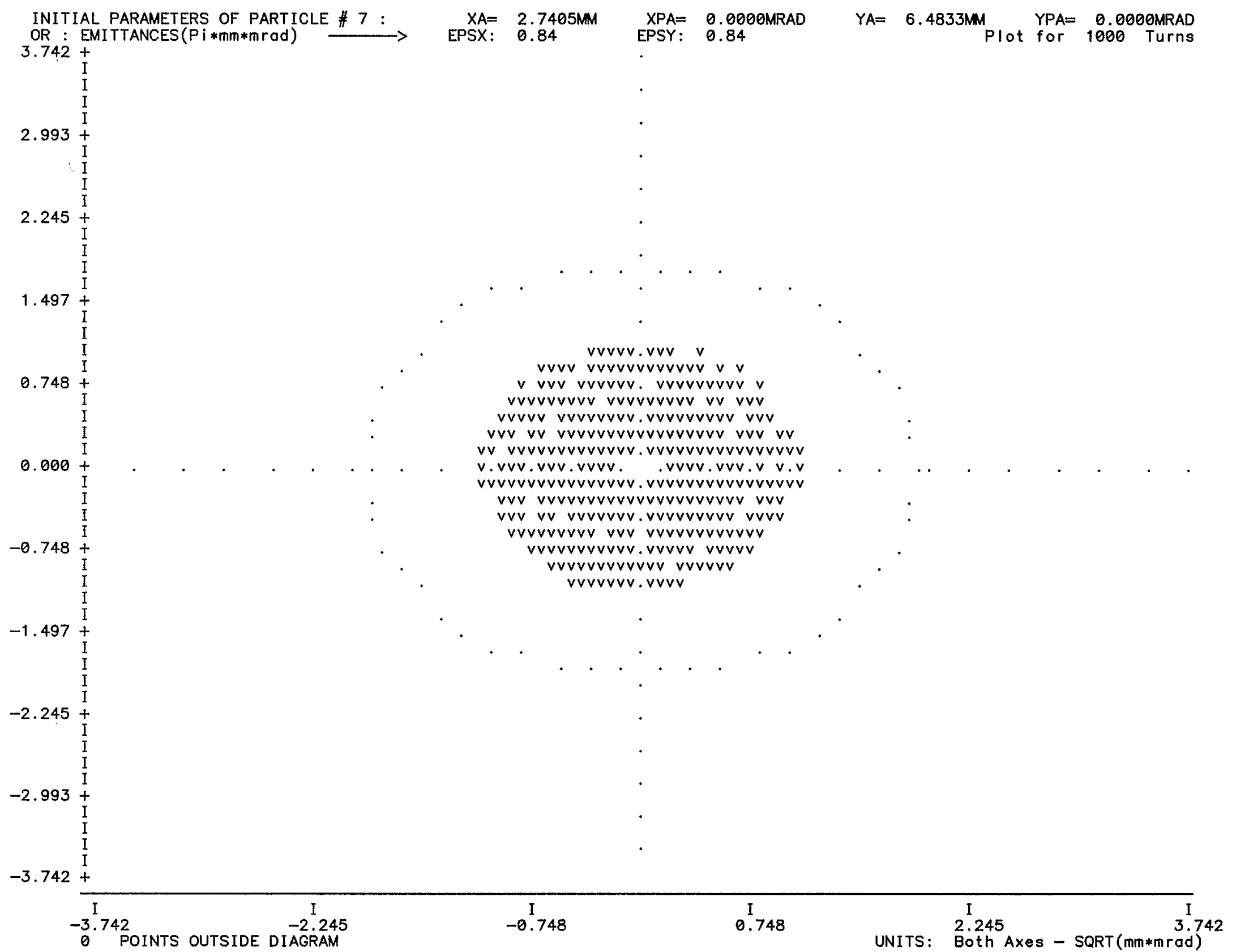

Figure 14. Particle trajectory in Floquet $\left(v, v^{\prime}\right)$ coordinates. Case \#2. 


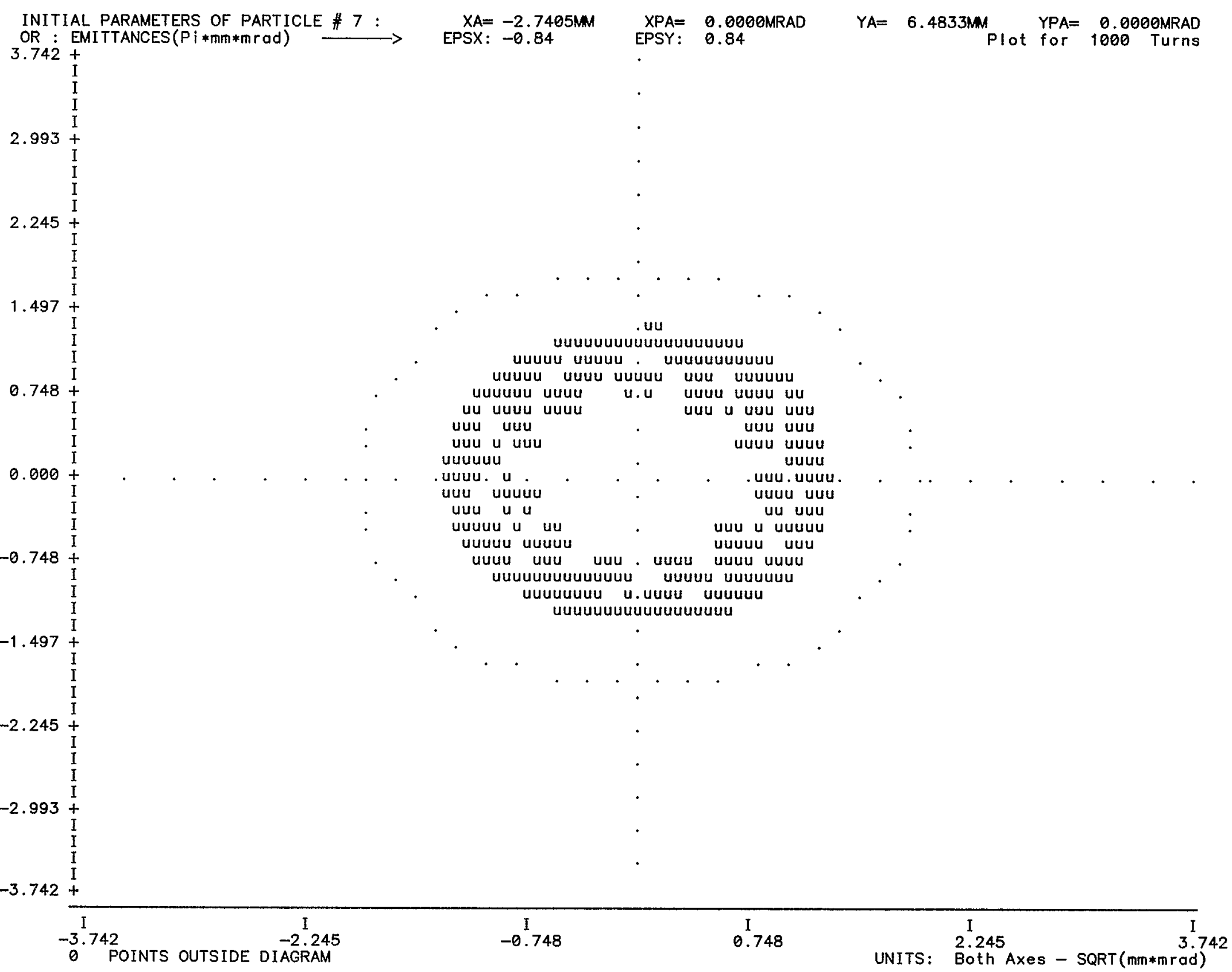

Figure 15. Particle trajectory in Floquet ( $u, u^{\prime}$ ) coordinates. Case \#4. 


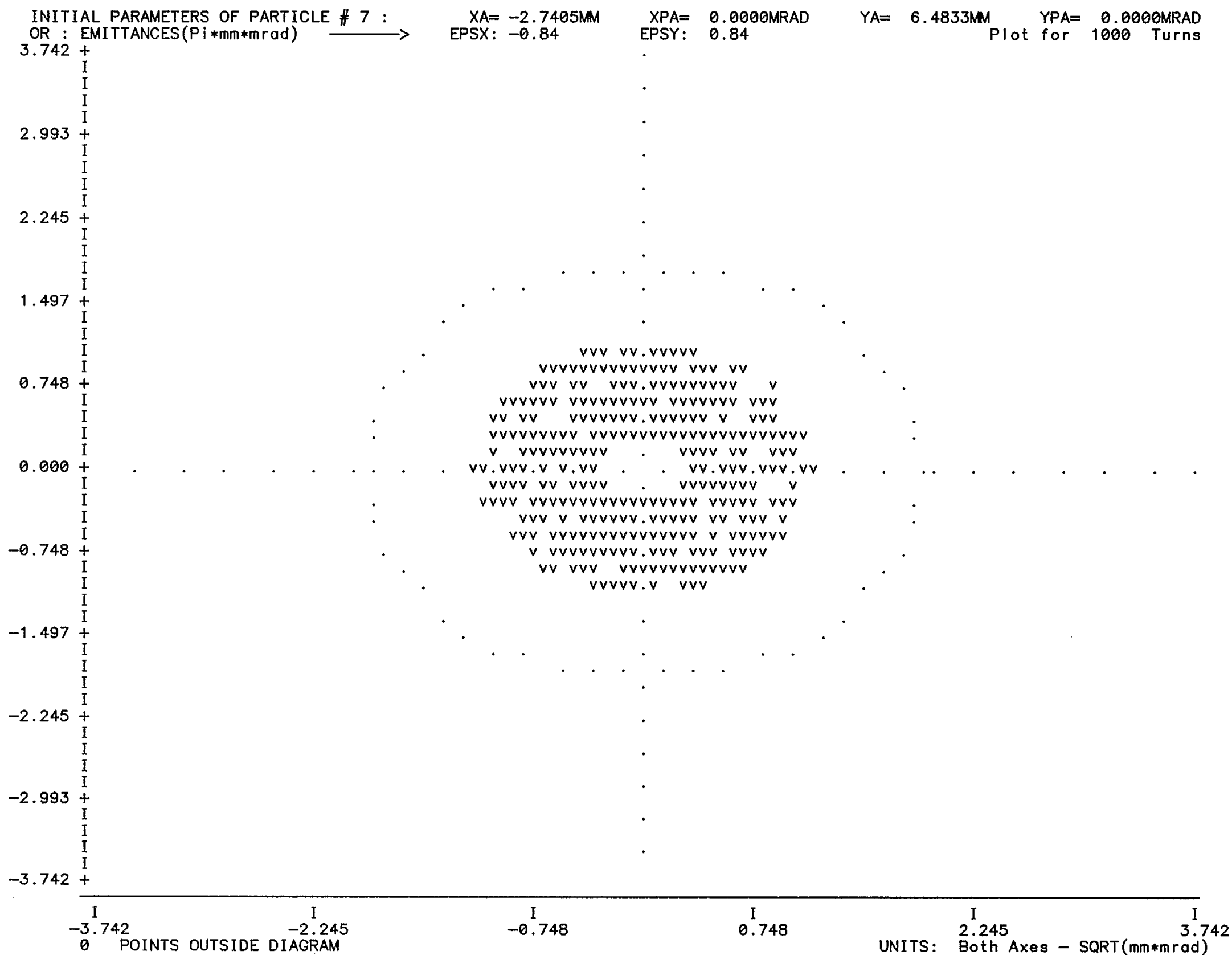

Figure 16. Particle trajectory in Floquet $\left(v, v^{\prime}\right)$ coordinates. Case \#4. 
Fig. 17 Horiz. and Vert. Emittance Case \# 2

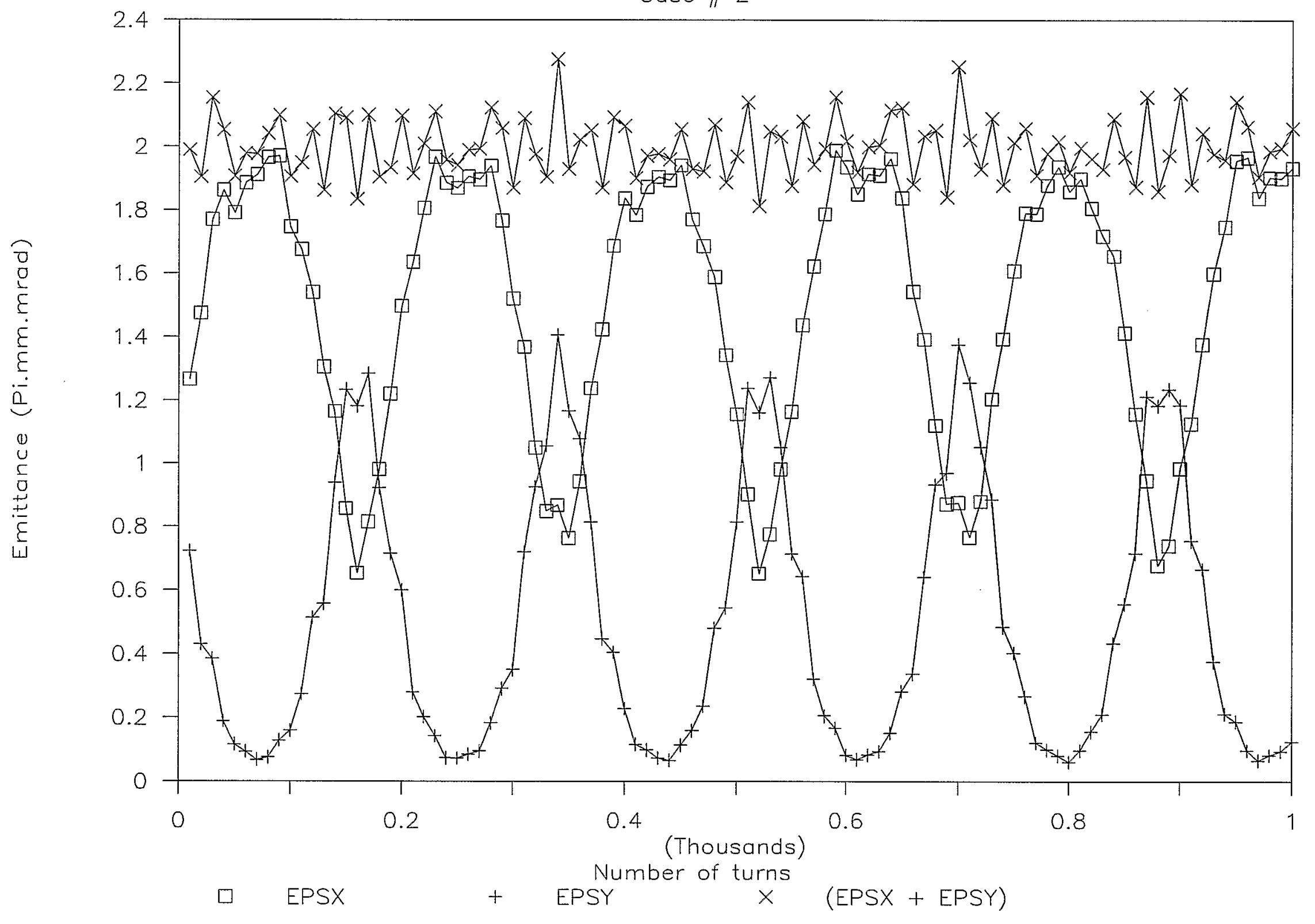


Fig. 18 Horiz. and Vert. Emittance Case \# 4

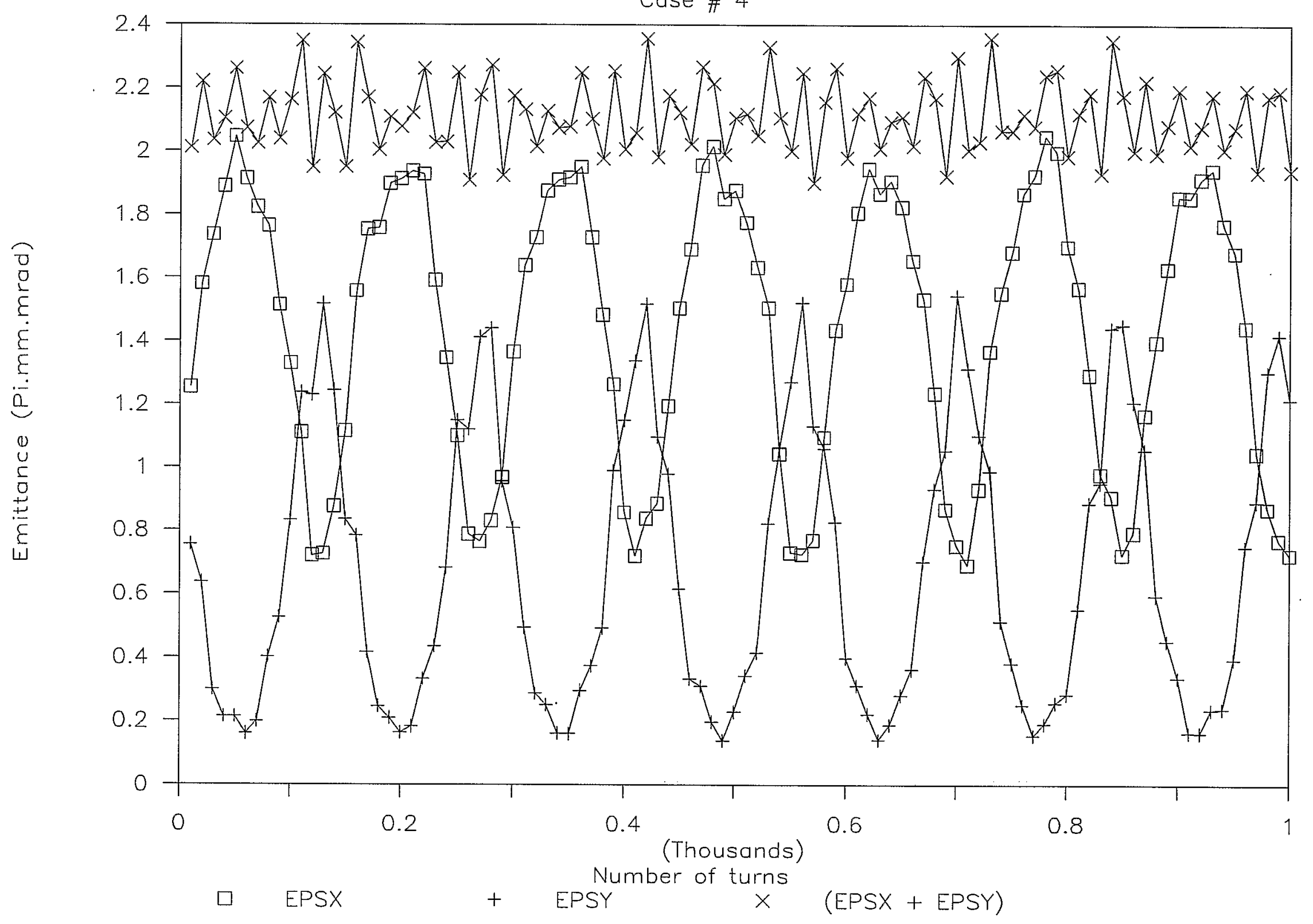


Fig. 19 Horiz. and Vert. Emittance Case \# 2, large amplitudes

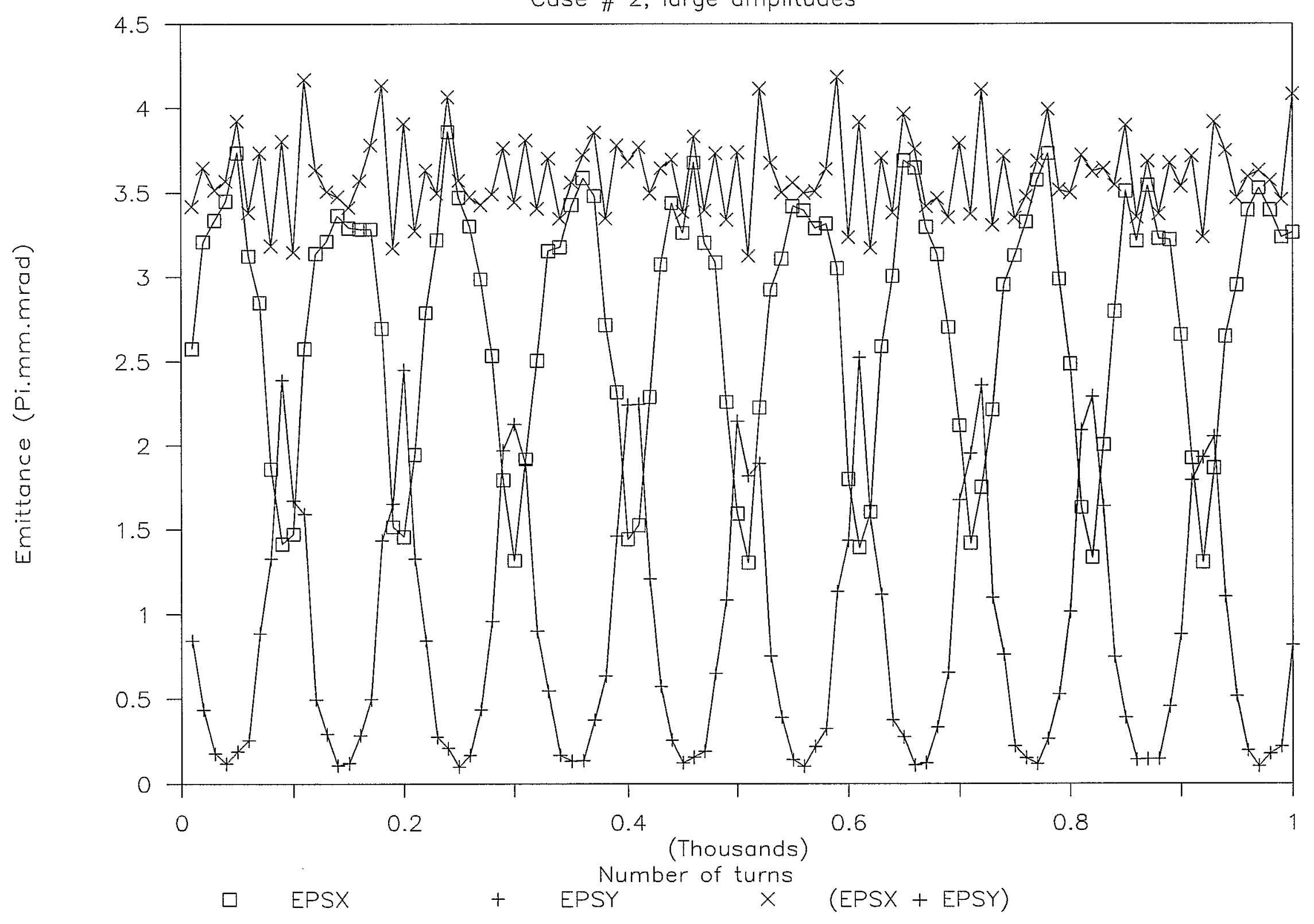


Fig. 20 Horiz. and Vert. Emittance

Case \# 4, large amplitudes

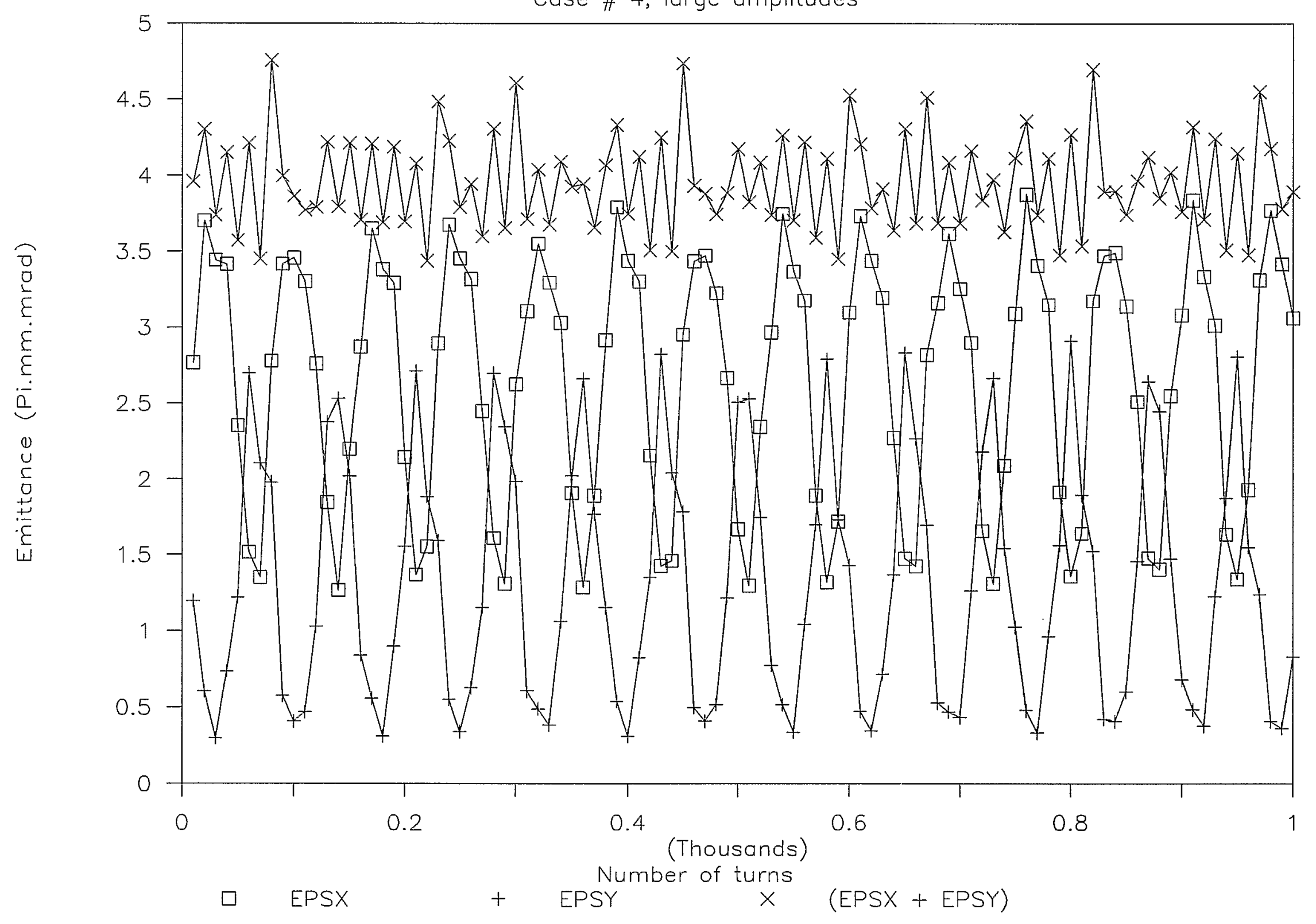




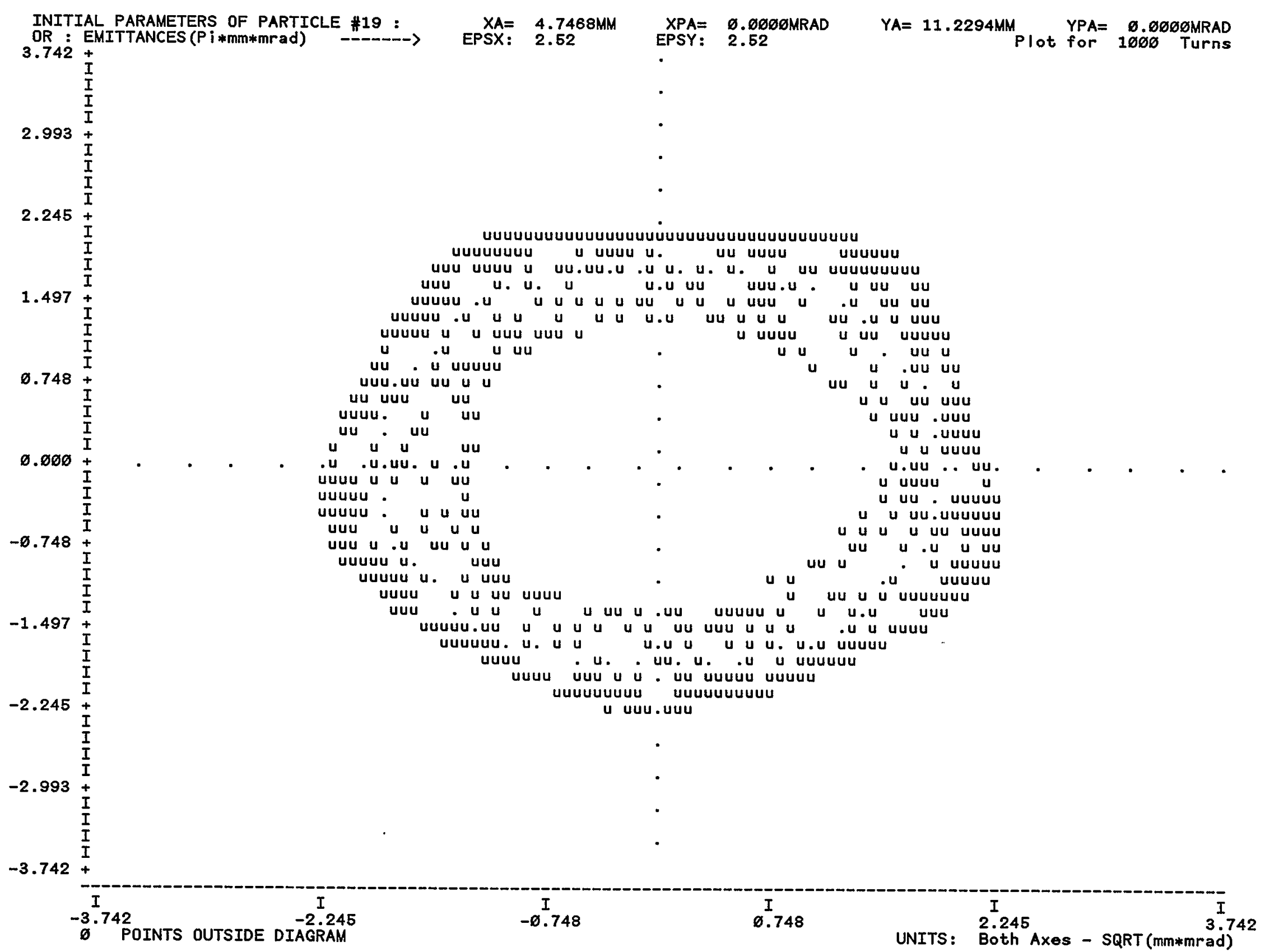

Figure 21. Particle trajectory in Floquet (u, $\left.u^{\prime}\right)$ coordinates. Case \# 2, amplitude close to dynamic aperture. 


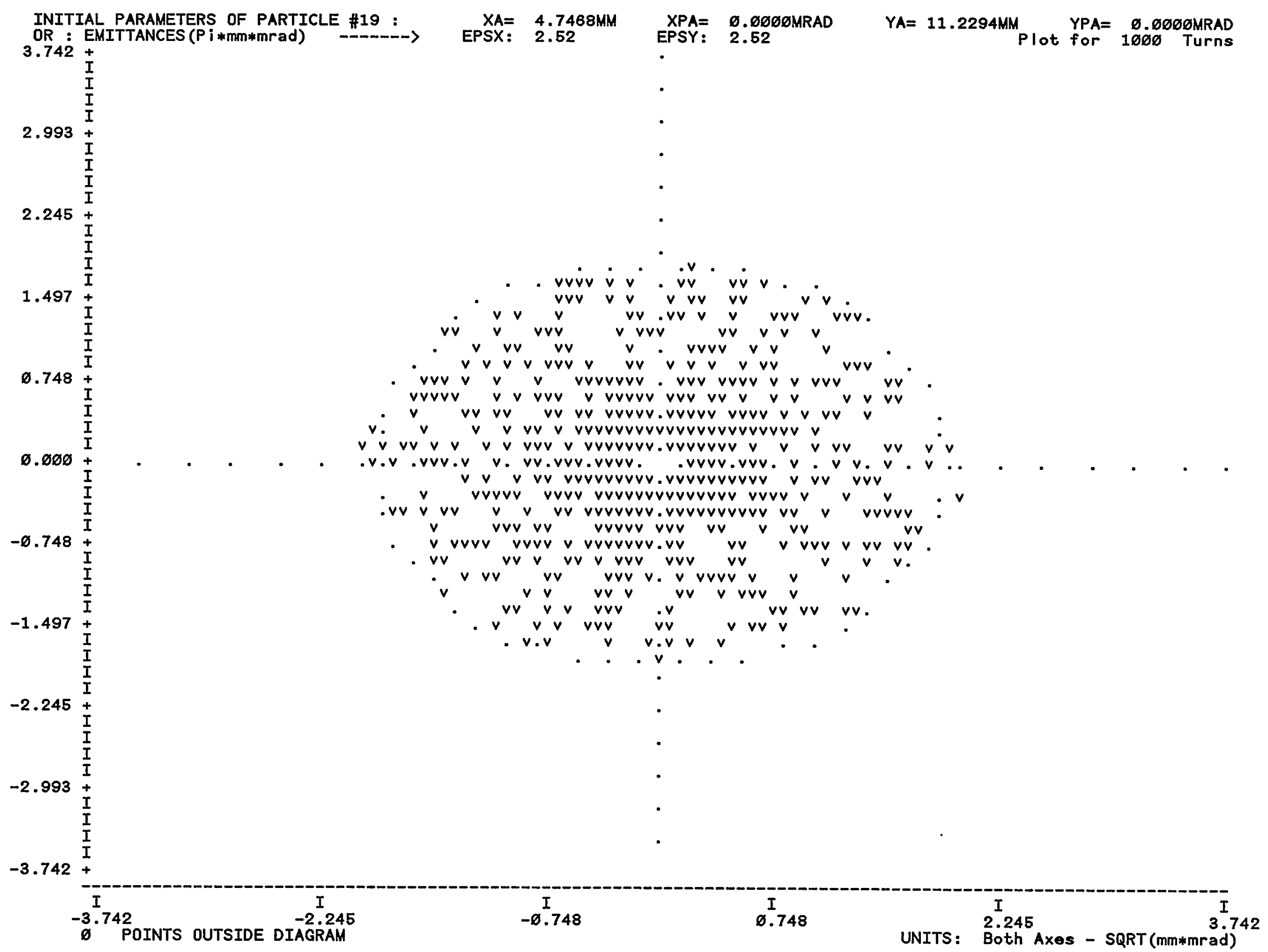

Figure 22. Particle trajectory in Floquet ( $\left.v, v^{\prime}\right)$ coordinates. Case \# 2, amplitude close to dynamic aperture. 


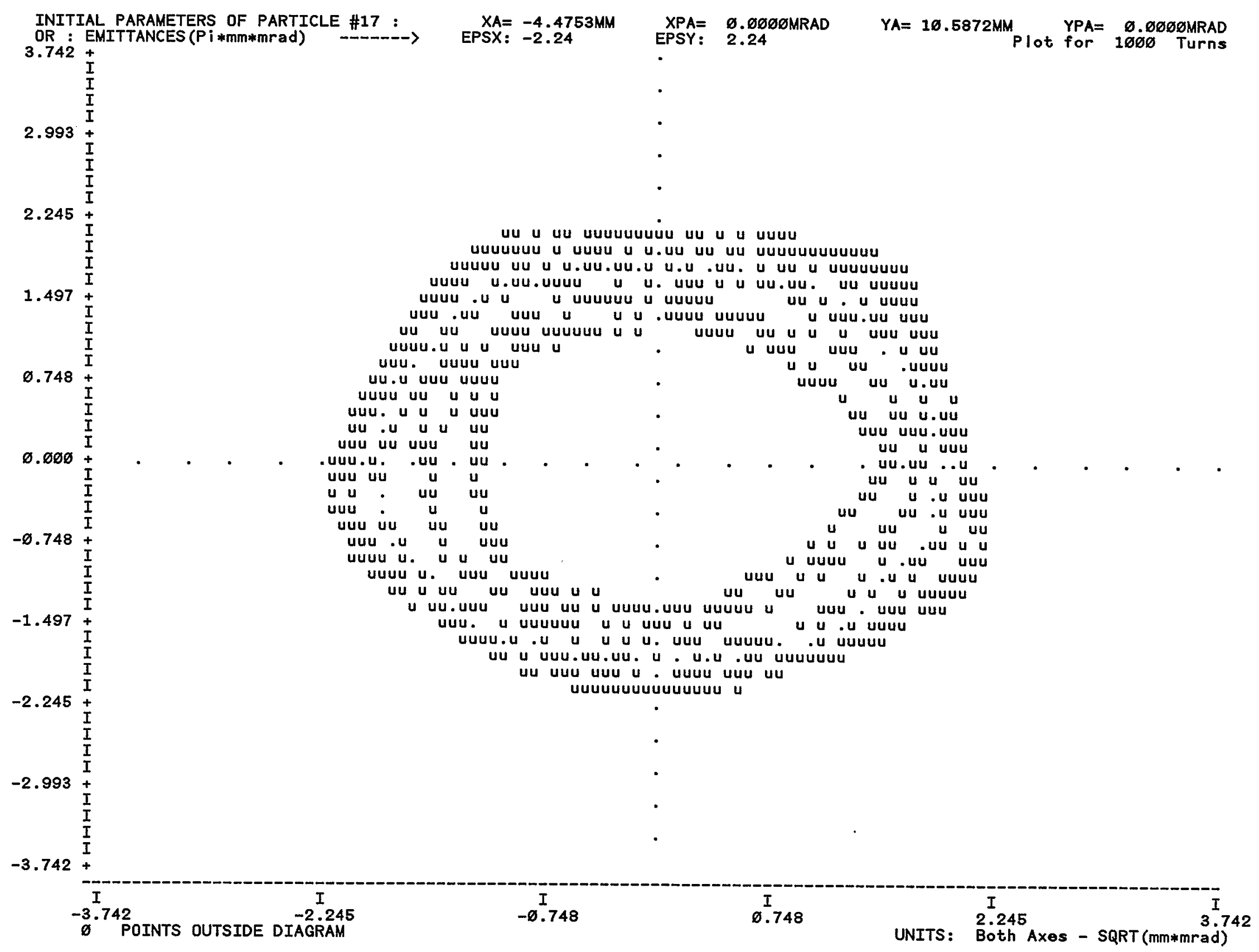

Figure 23. Particle trajectory in Floquet $\left(u, u^{\prime}\right)$ coordinates. Case \# 4, amplitude close to dynamic aperture. 


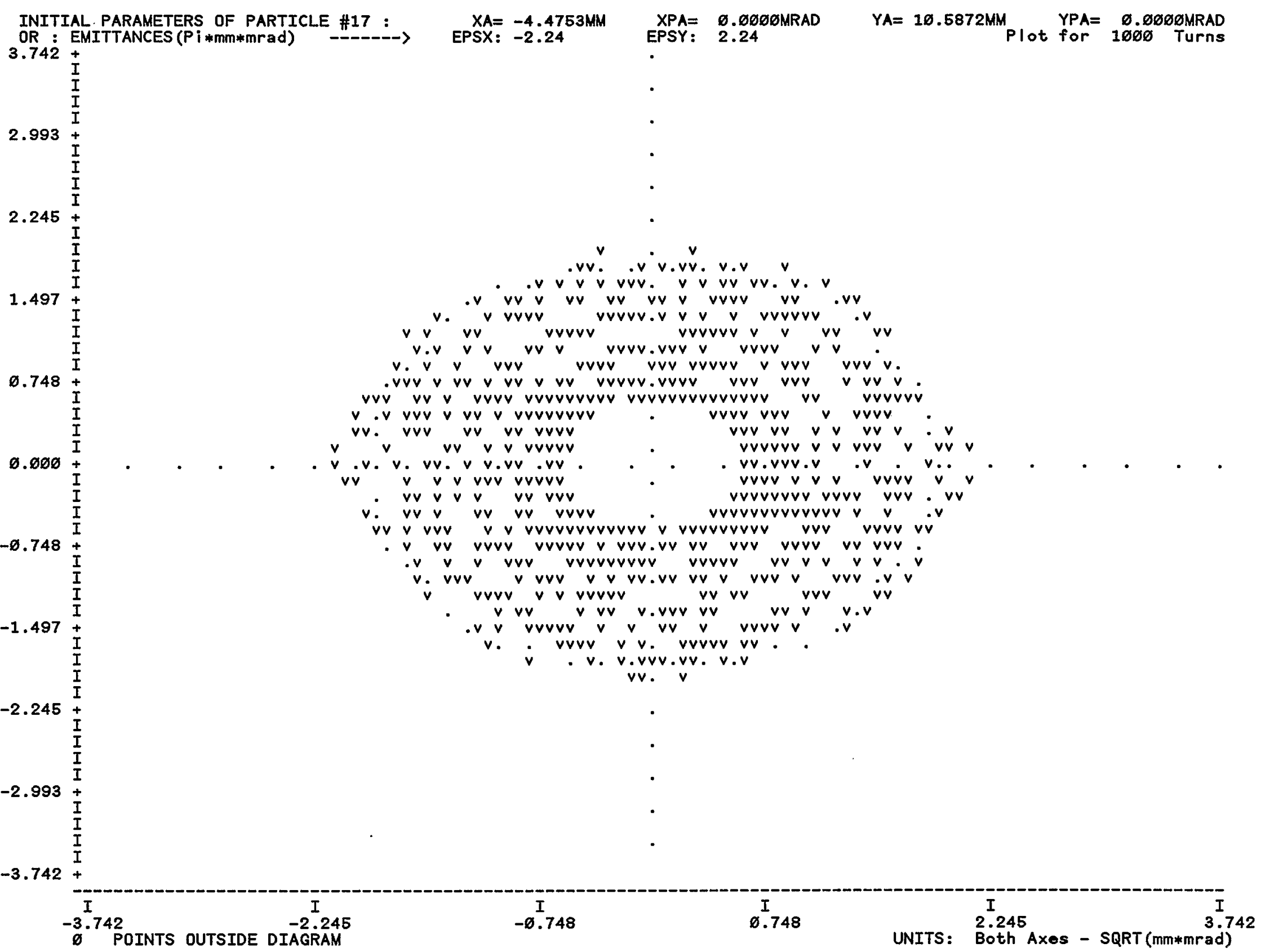

Figure 24. Particle trajectory in Floquet $\left(v, v^{\prime}\right)$ coordinates. Case \# 4 , amplitude close to dynamic aperture. 


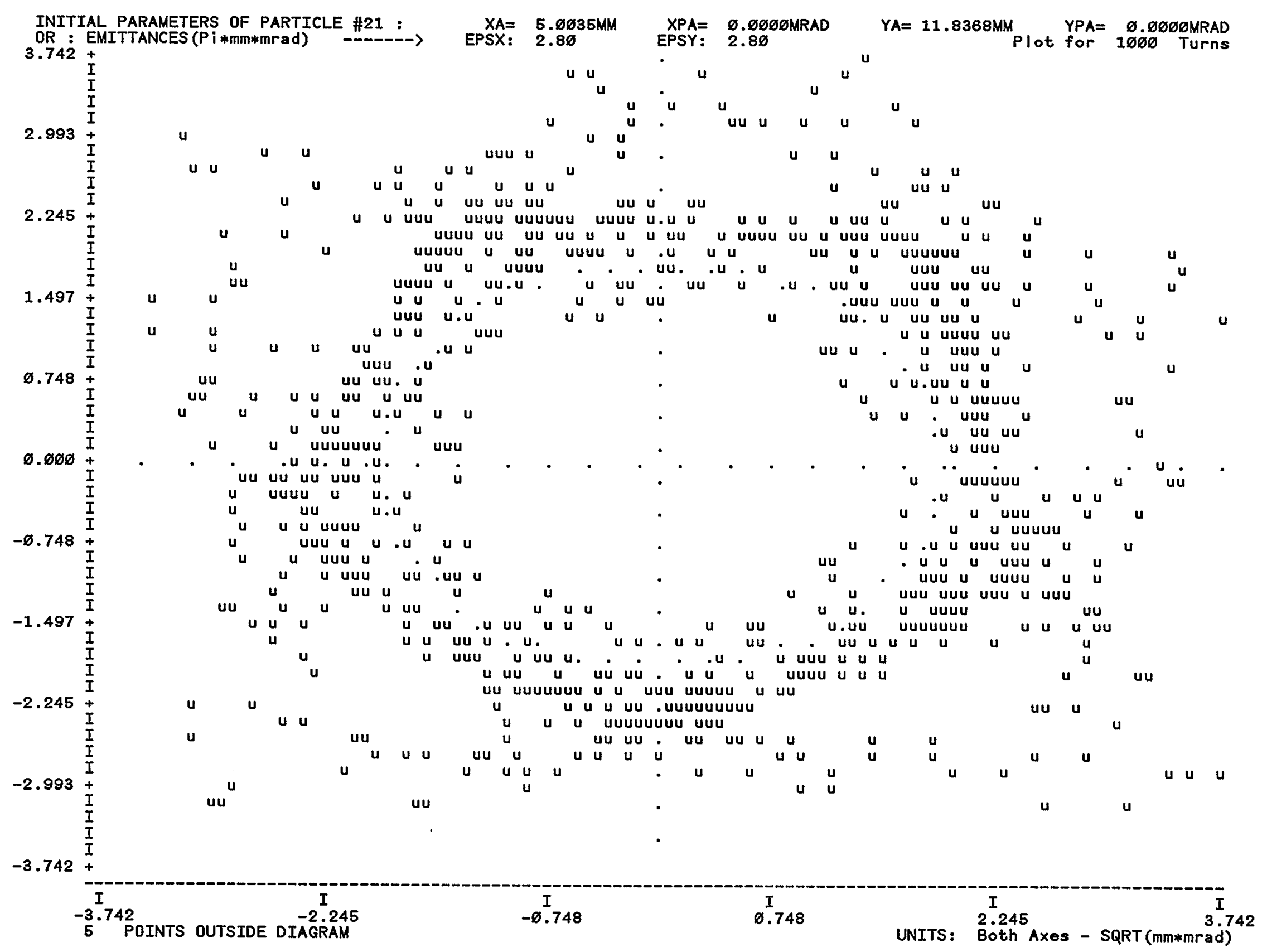

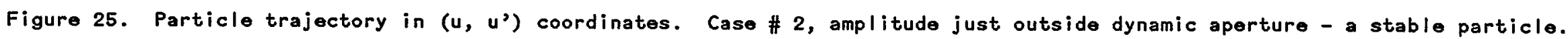




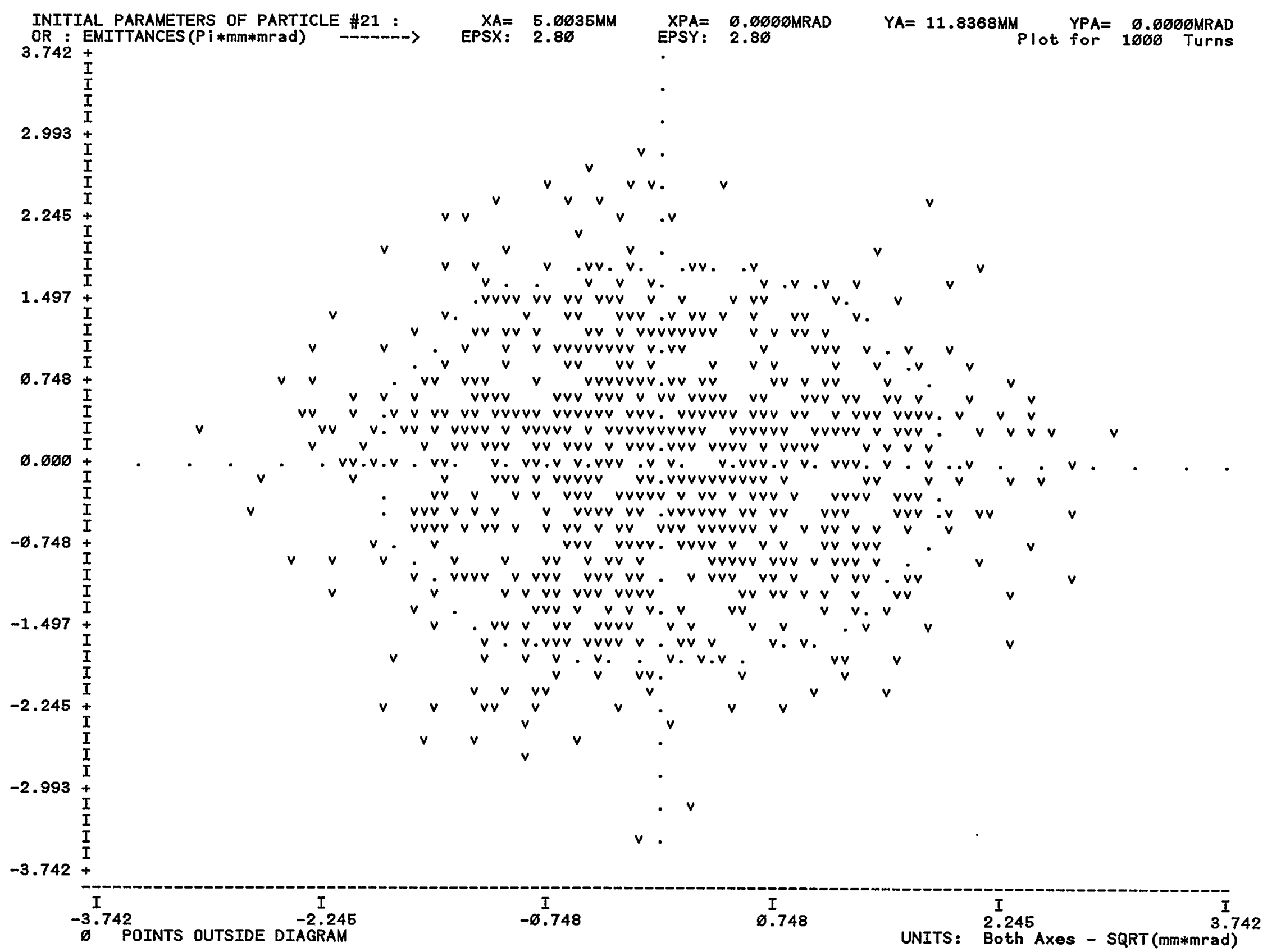

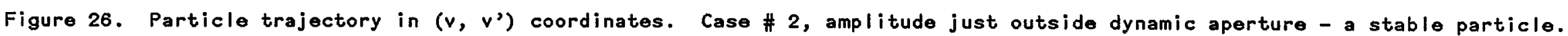




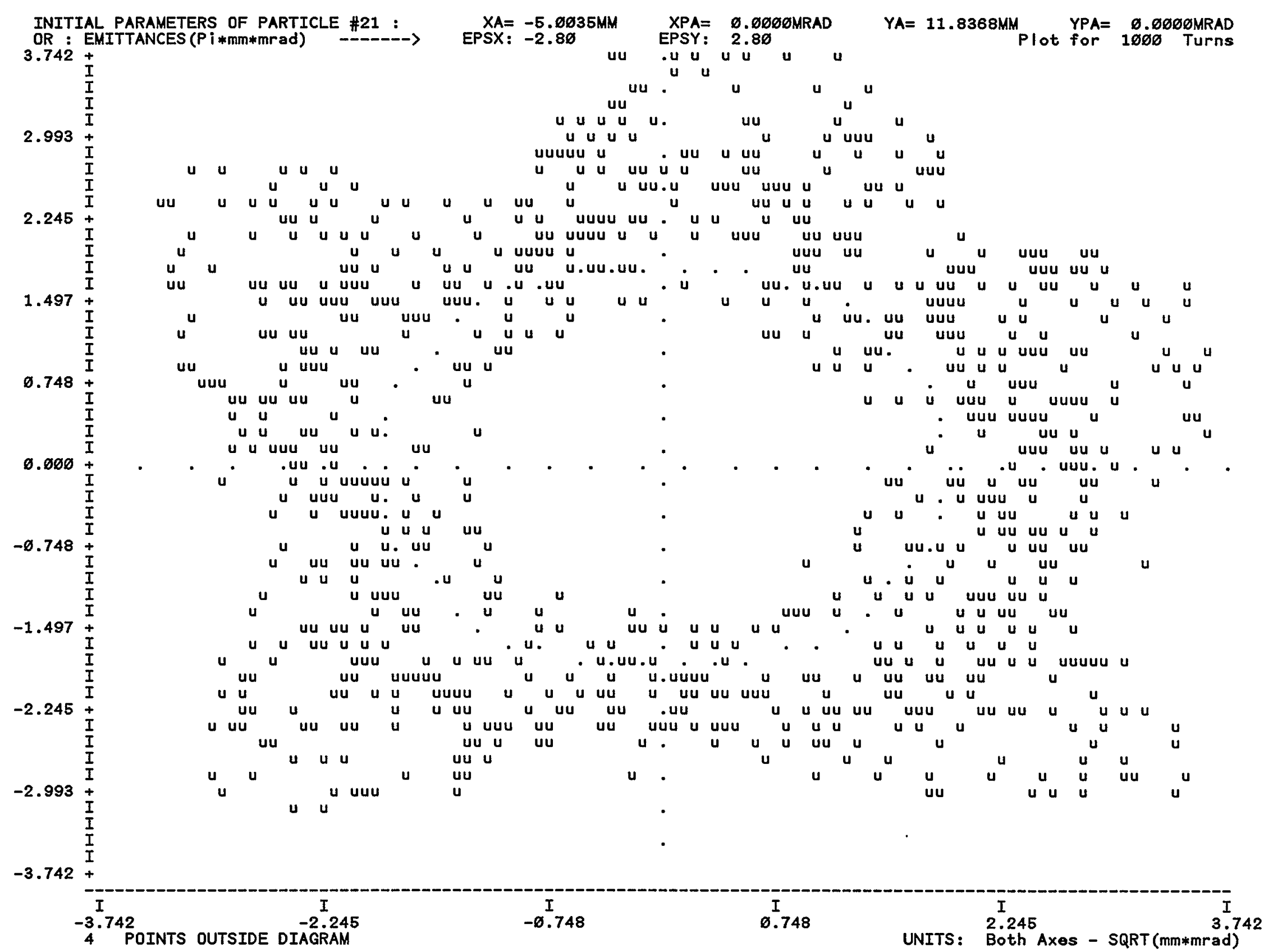

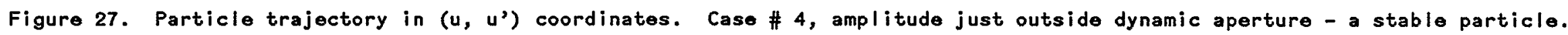




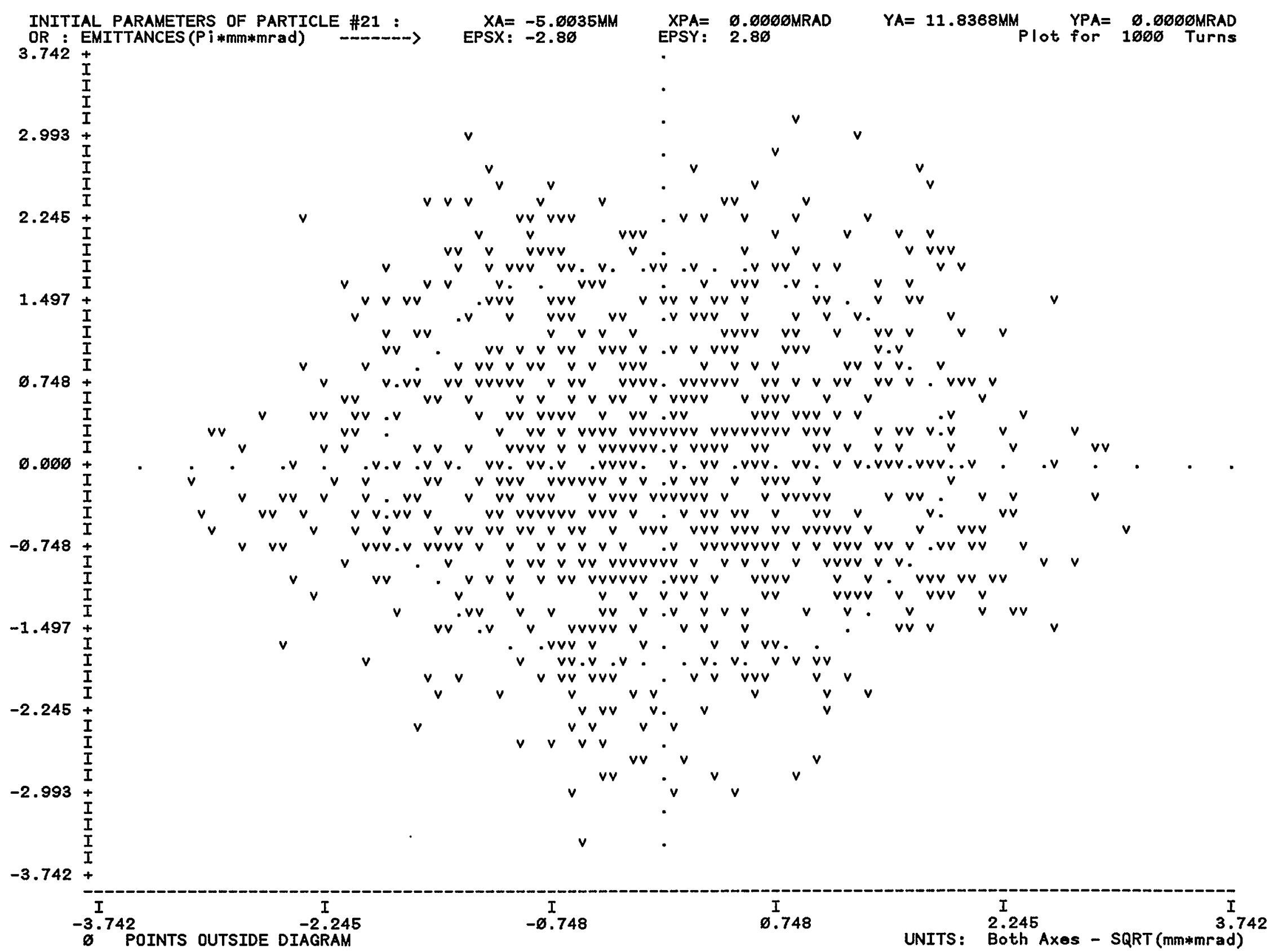

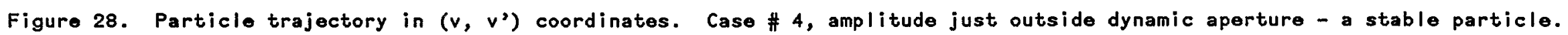




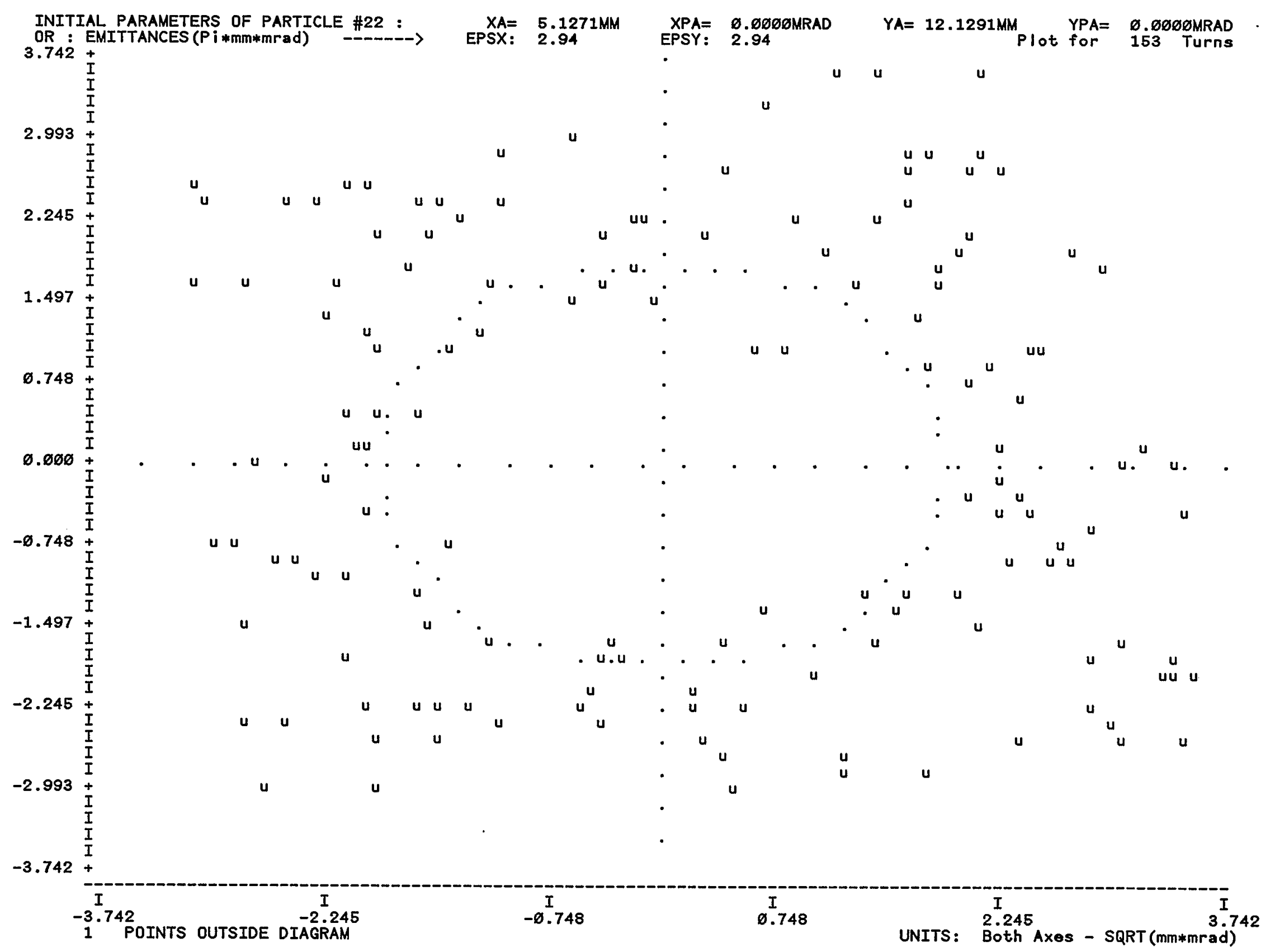

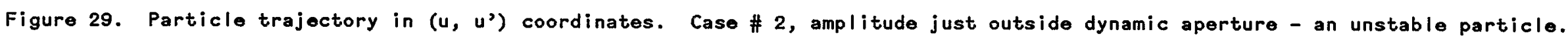




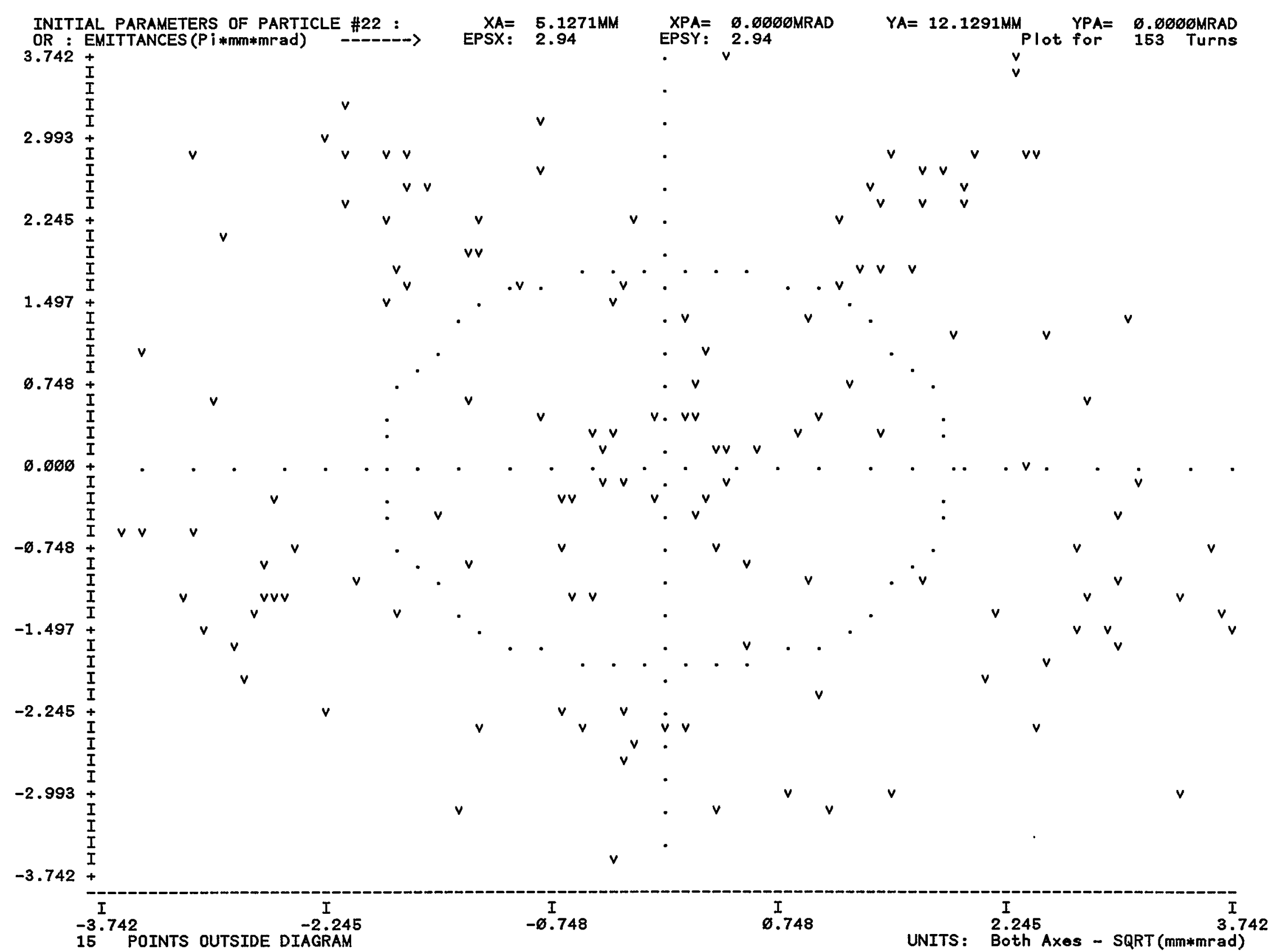

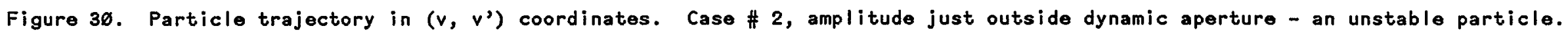




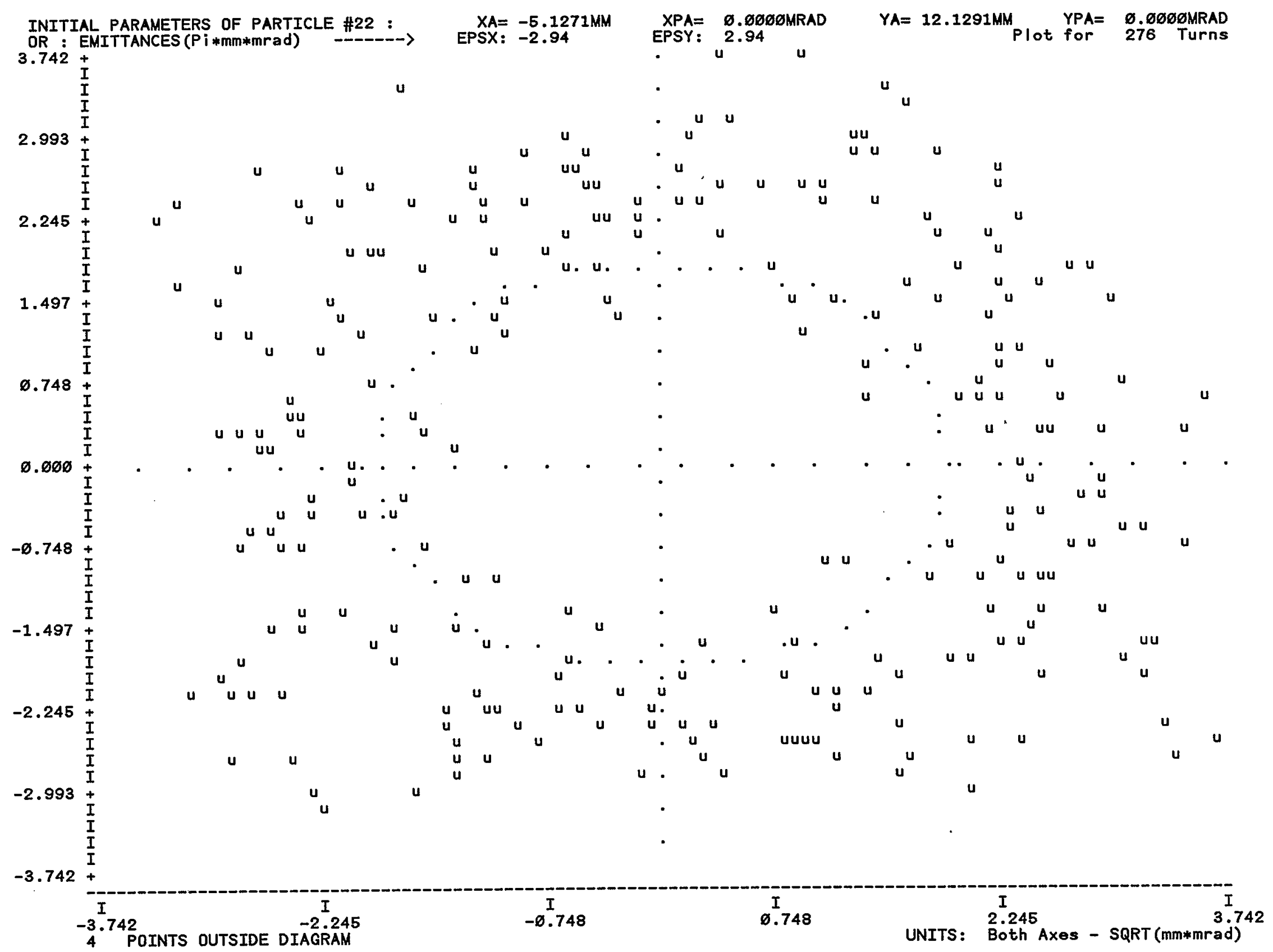

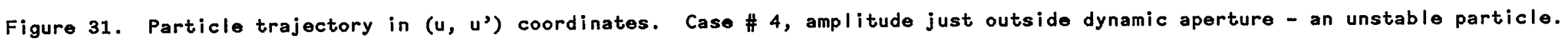




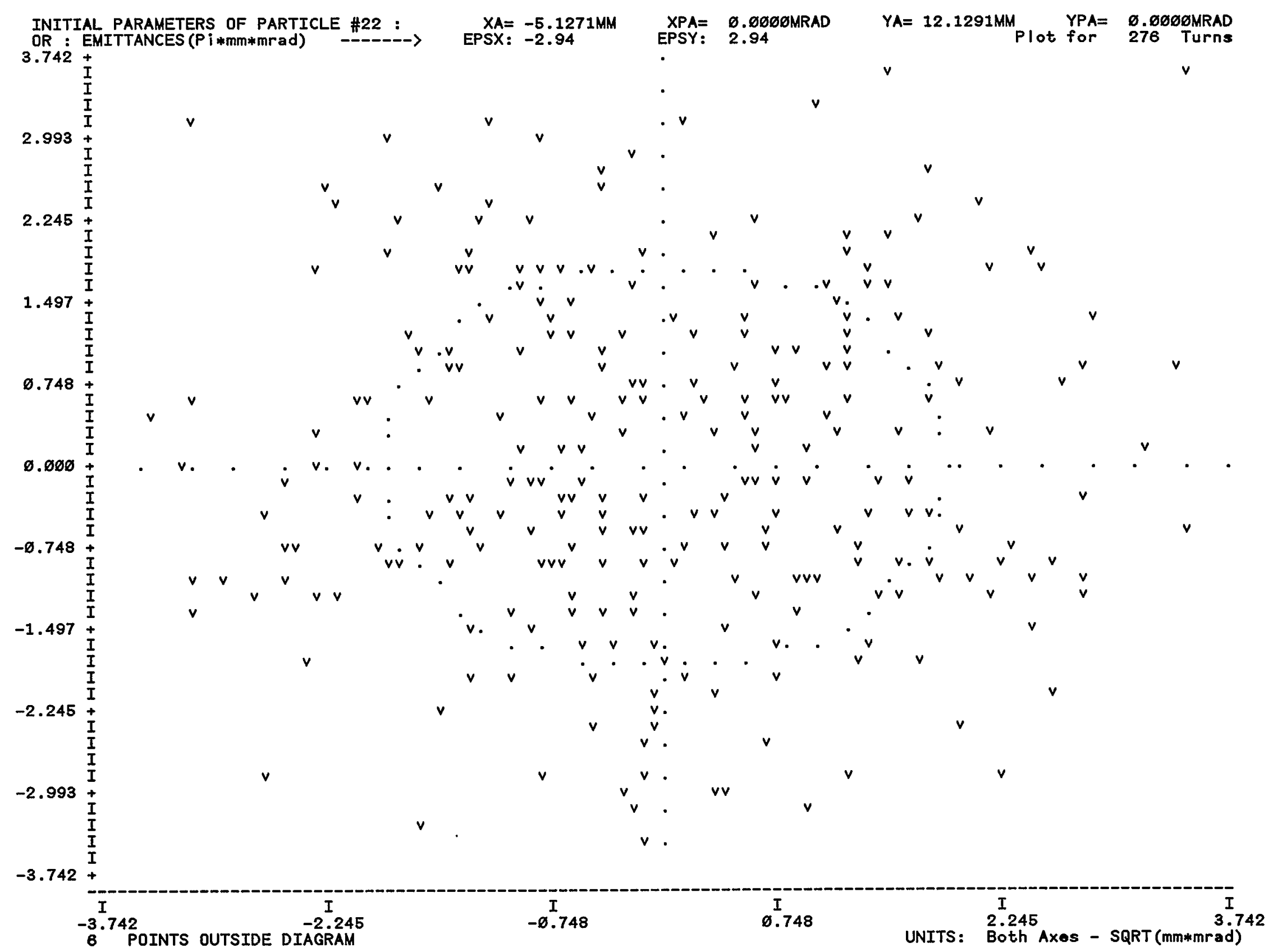

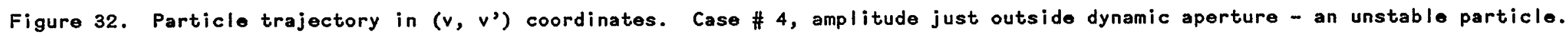




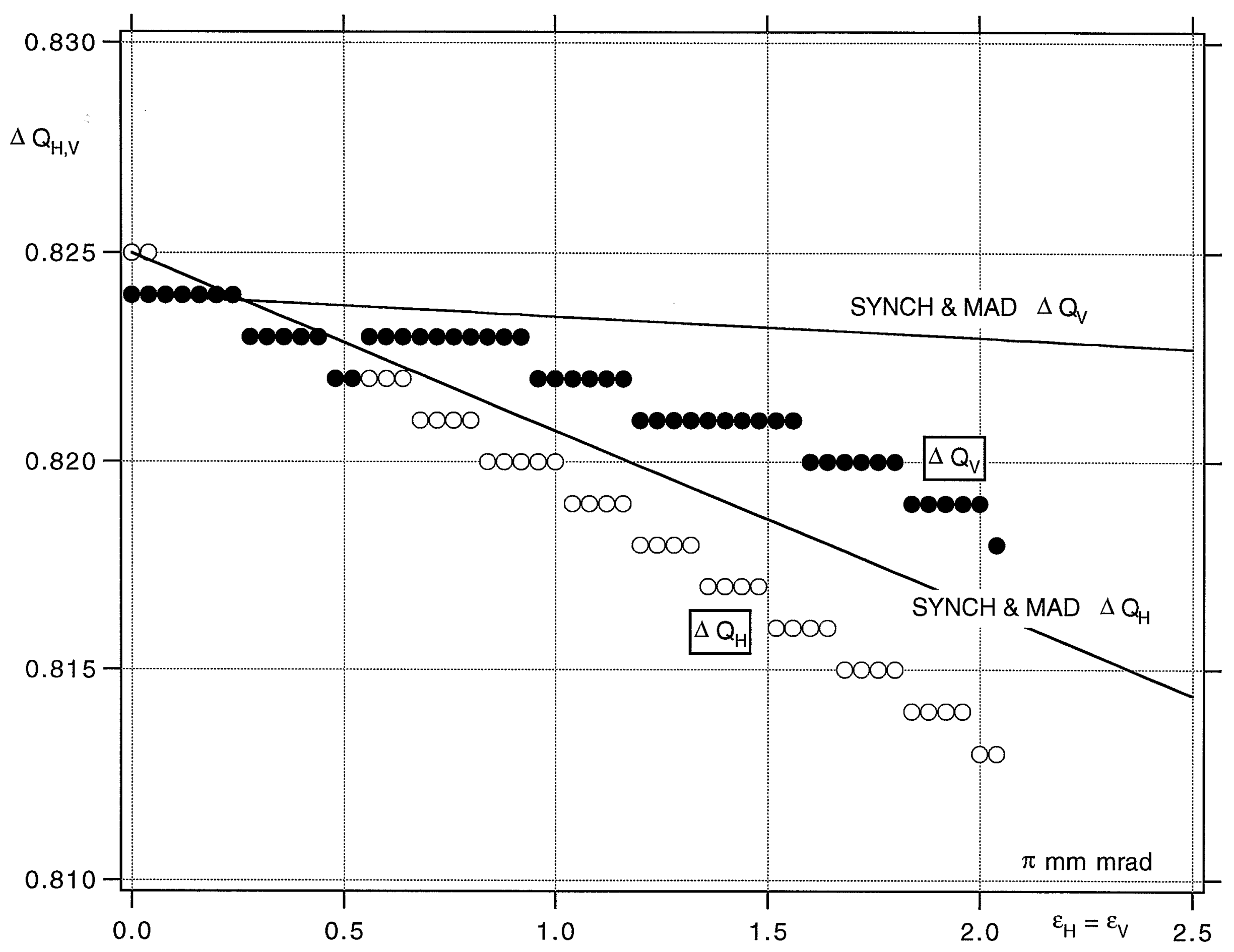

Fig. 33 Betatron Tune-Shifts vs. Emittance for case \#2 


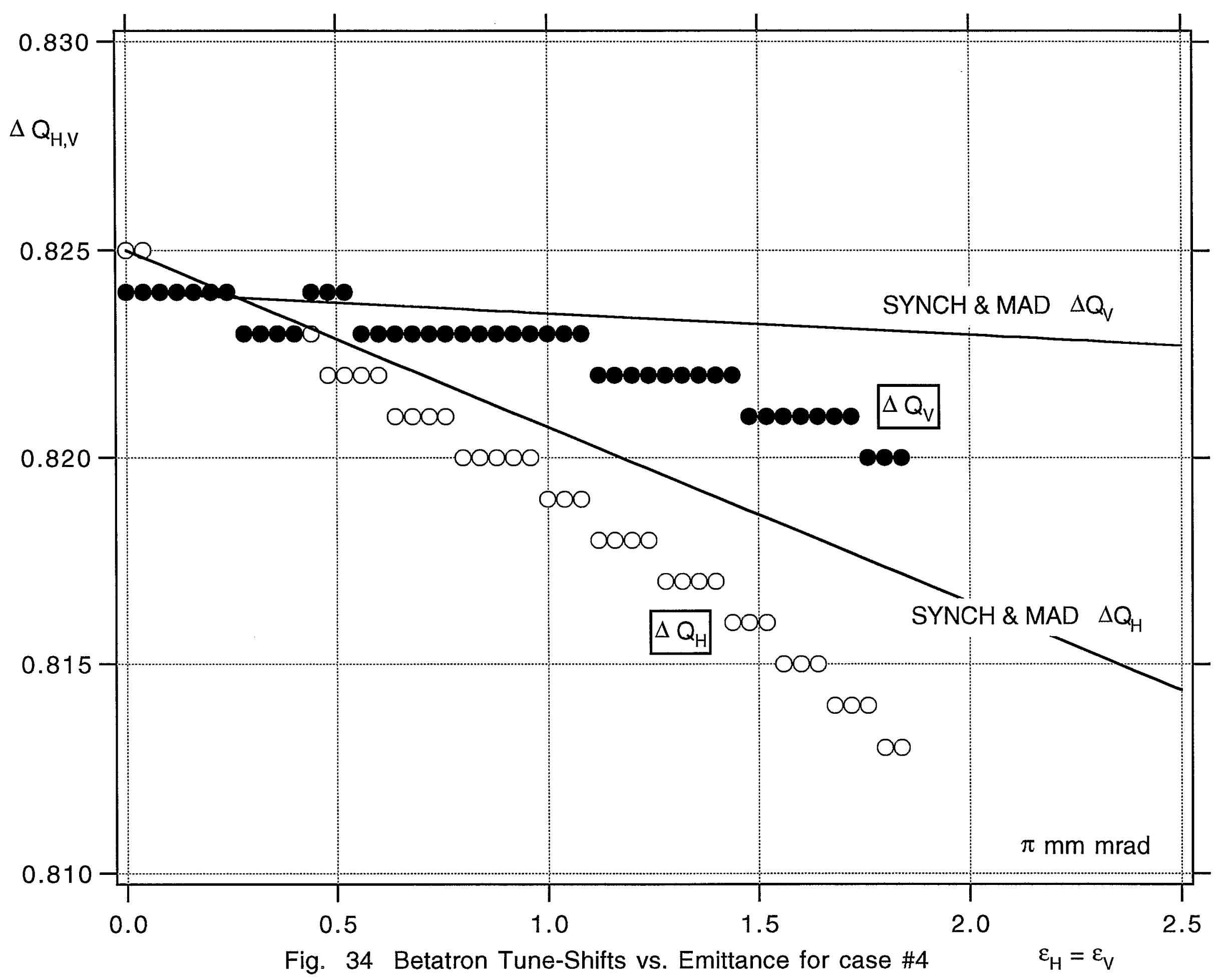


***** PARTICLE $=20 \quad * * * * *$

BETATRON OSCILLATION FREQUENCY SPECTRUM

$1.000+$

DNUEX $=0.824000$

DNUEY $=0.825000$

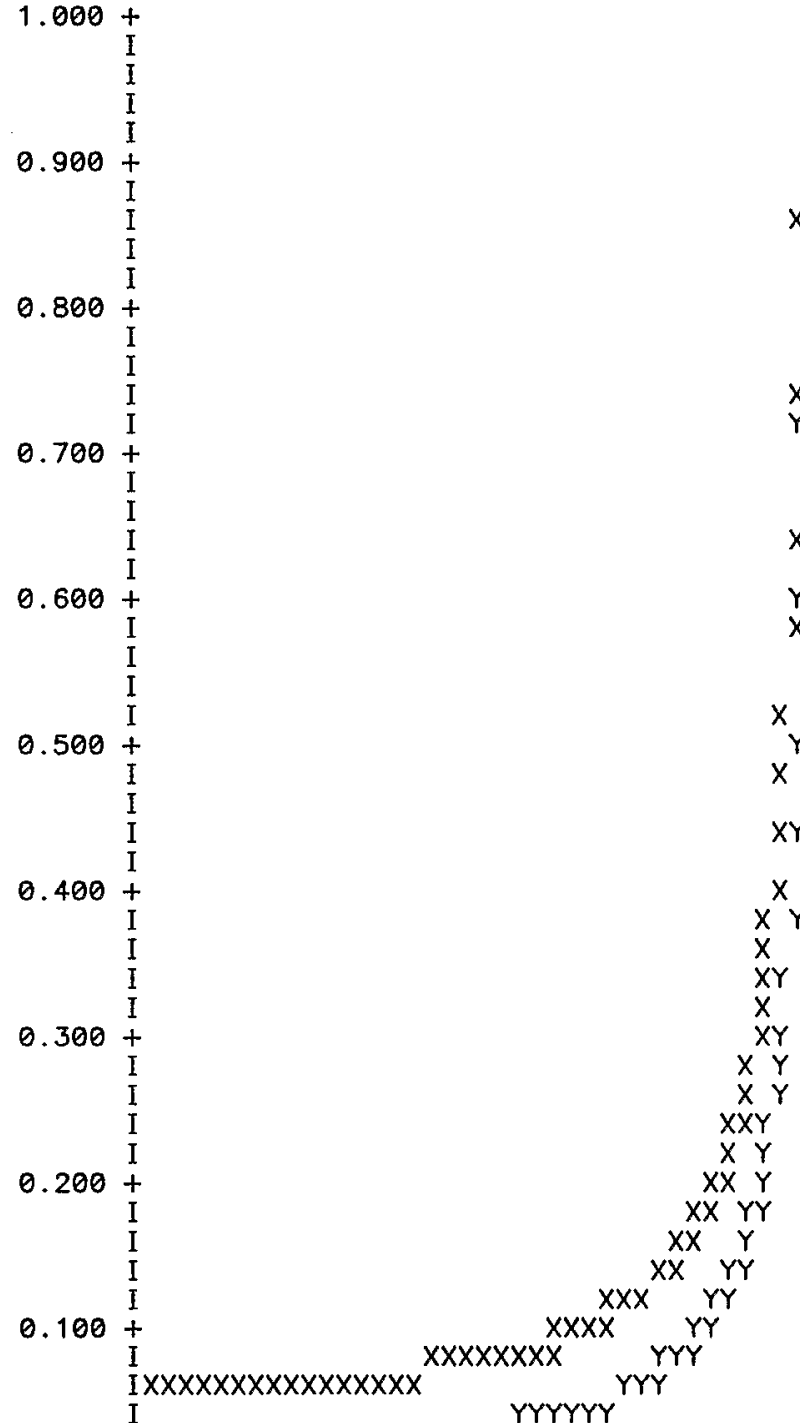

0.000 I +YYYYYYYYYY YYYYYYYYYYYY

Y

$x \times$

$Y$

$X$

$Y$
$X$

$\dot{Y} x$

$X^{Y} Y_{Y}^{X}$

$X Y$

$x_{X}^{X} Y X X$

$\begin{array}{ll}X Y \\ X & Y \\ X & Y \\ X & Y\end{array}$

$\begin{array}{ll}X Y & Y \\ X & Y X\end{array}$

$\begin{array}{rr}X Y & X \\ X & Y X\end{array}$

$Y \quad Y X X$

$\begin{array}{ll}X X Y & Y X X \\ X Y Y & Y X\end{array}$

$\begin{array}{ll}X X Y & Y X \\ X Y & Y X\end{array}$

$X X Y Y Y X X$

$\begin{array}{ll}X X X Y & Y X \\ X X Y & Y Y X\end{array}$

$\begin{array}{ll}Y Y X & Y X X \\ Y X X & Y X X \\ Y Y X & \end{array}$

$Y X X$
$Y X X$
$Y Y X X$

YYXX

\section{YYYYYYYY} $X$ SYYYYYYYYYY $x$

$\times \times$

$\times \times$

$x$

$x$
$x$

$\begin{array}{ll}x & x \\ x & x\end{array}$

$x$

$\begin{array}{ll}x & x \\ x & x \\ x & x\end{array}$

$\begin{array}{ll}x & x \\ x & x \\ x & x\end{array}$

$\begin{array}{ll}x & x \\ x & x \\ x & x\end{array}$

$\begin{array}{rl}x & x \\ x & x x \\ x & x x x\end{array}$

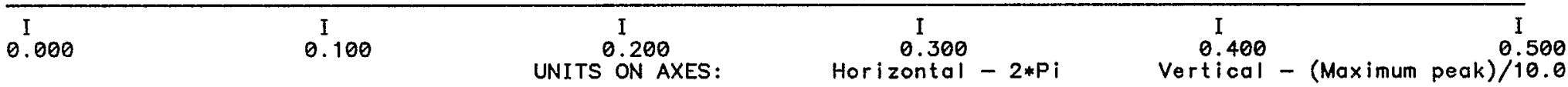

Figure 35. Betatron spectrum for particle no. 20 of case $\# 3$. Truncated at $1 / 10$ th of the maximum peak. 
***** PARTICLE $=21 \quad * * * * *$

BETATRON OSCILLATION FREQUENCY SPECTRUM

$1.000+$

I
I
I
I
I
I
I
I
$0.800+$

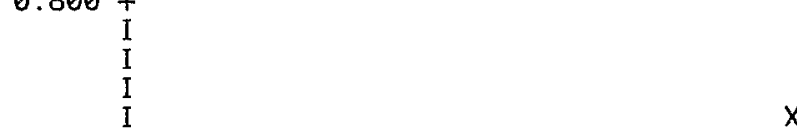

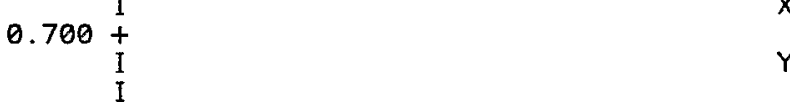

$0.600 \stackrel{\text { I }}{\mathrm{I}}$

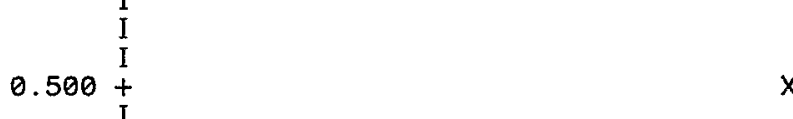

$\begin{array}{rl}0.500+ & x \\ \text { I } & x \\ \text { I } & x \\ I & x\end{array}$

$0.400+\frac{I}{1} \quad Y X X Y$

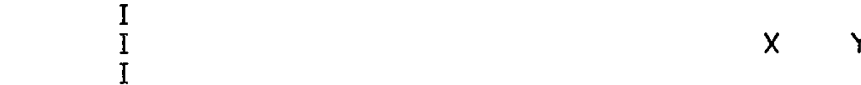

$\begin{array}{rrr}1 & r \\ I & X & Y \\ I & X & Y \\ I & Y X & Y \\ I & X & Y\end{array}$

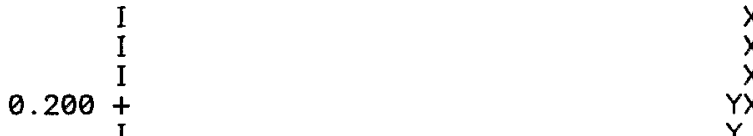

0.100

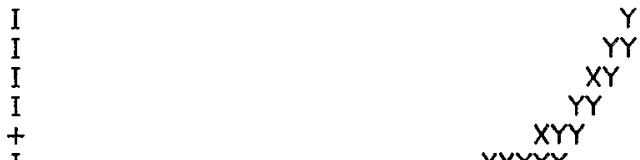

I $X X X X X Y Y Y Y Y$

IXXXXXXXXXXXXXXXXXXXXYYYYYYYY

$0.000+Y$ IYYYYYYYYYYYYYYYYYYYY

$x$

Y
DNUEX $=0.651000 \quad$ DNUEY $=0.825000$

$\mathrm{X}$

X

$X^{Y}$

$Y Y X$

$Y$
$Y$$x^{X}$

$\begin{array}{ll}Y & X \\ Y & x \\ Y Y & X \\ Y & x\end{array}$

$\begin{array}{llll}X & & X & X \\ Y & & X & X \\ Y & X & X \\ Y Y & X & X & X\end{array}$

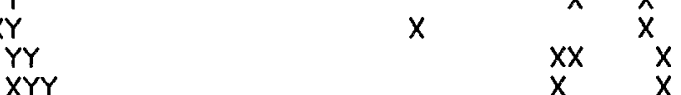

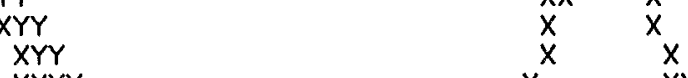

$\underset{X X Y Y}{X Y Y} \quad x \quad x \quad x \quad x$

$\begin{array}{llll}X Y Y Y Y & X & X & X X \\ X X Y Y Y Y & X & X\end{array}$

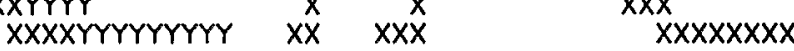

XXXXXXXXYYYYYYYYYYYYYYYYYYYYYYYYYYYYYYYXXXXXXXXXXXXXXXXXX

XX

Y YYYYYYYYYYYYYYYYYY

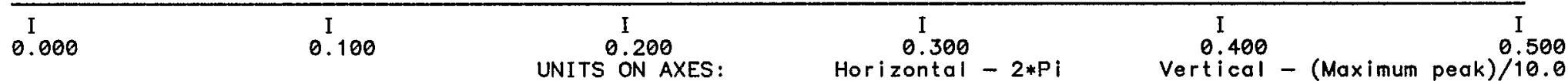

Figure 36. Betatron spectrum for particle no. 21 of case \#3. Truncated ot $1 / 10$ th of the maximum peak. 


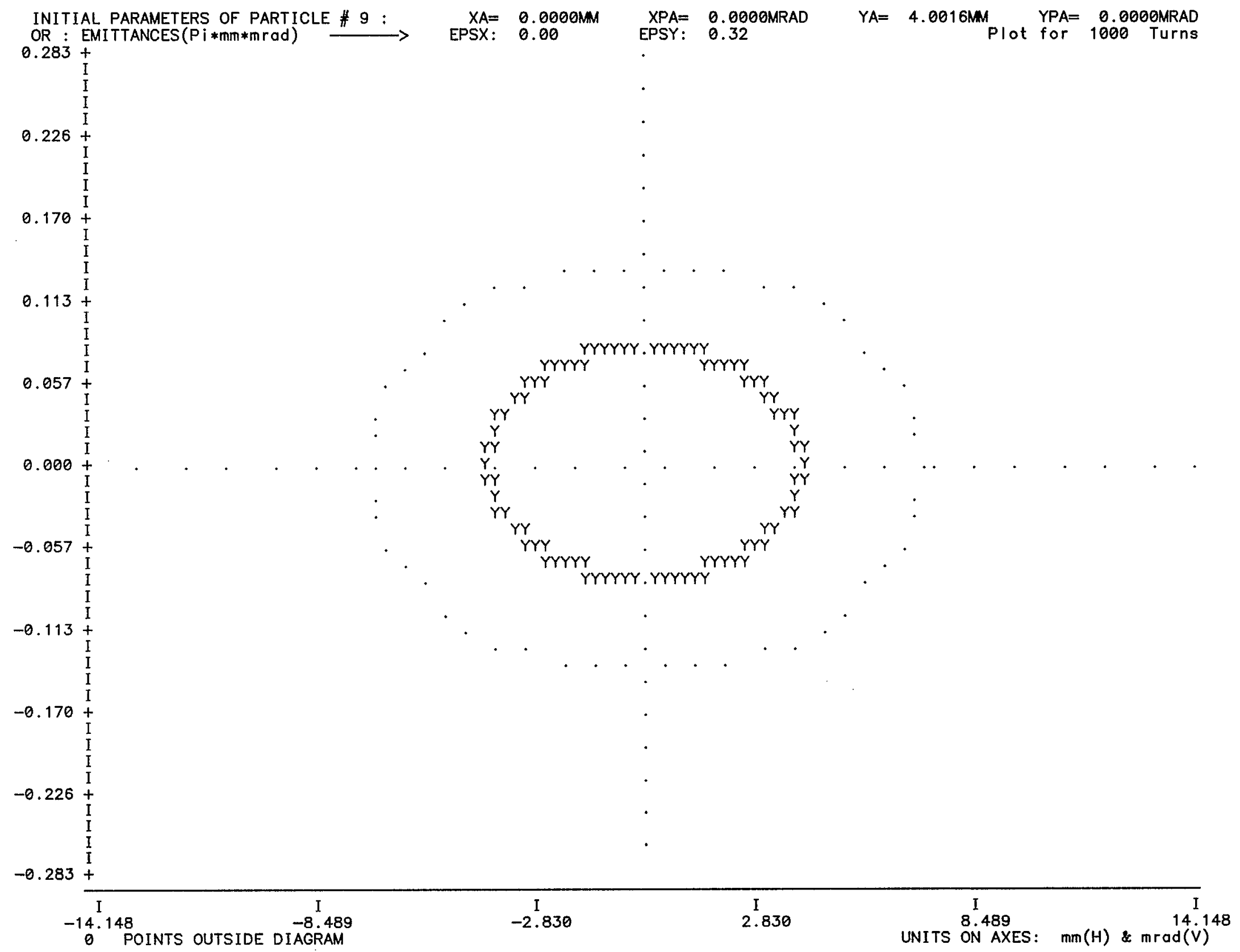

Figure 37. Particle trajectory in $\left(Y, Y^{\circ}\right)$ coordinates. Case \#3, small amplitude. 


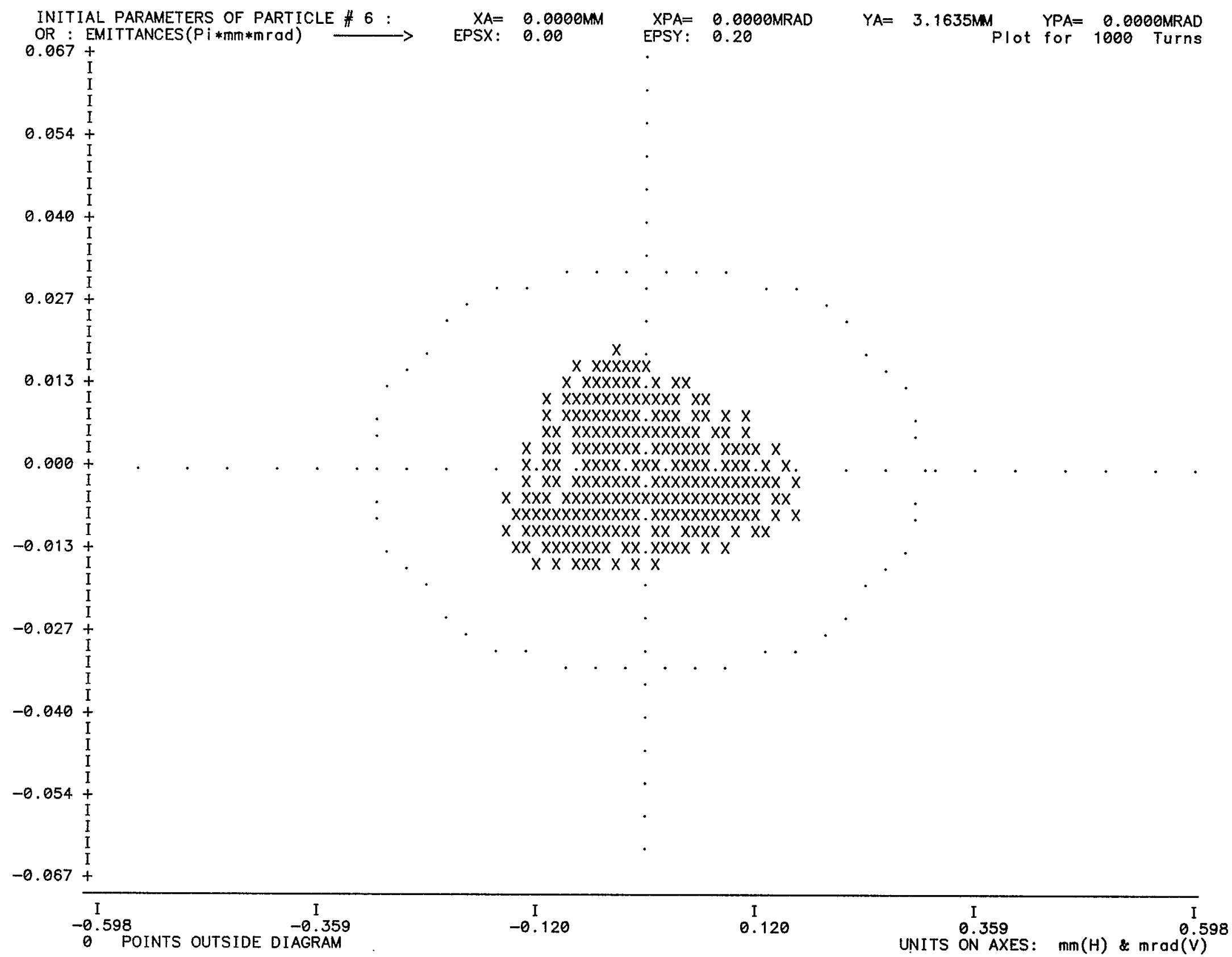

Figure 38. Particle trajectory in $\left(X, X^{\prime}\right)$ coordinates. Case \#, particle no. 6 


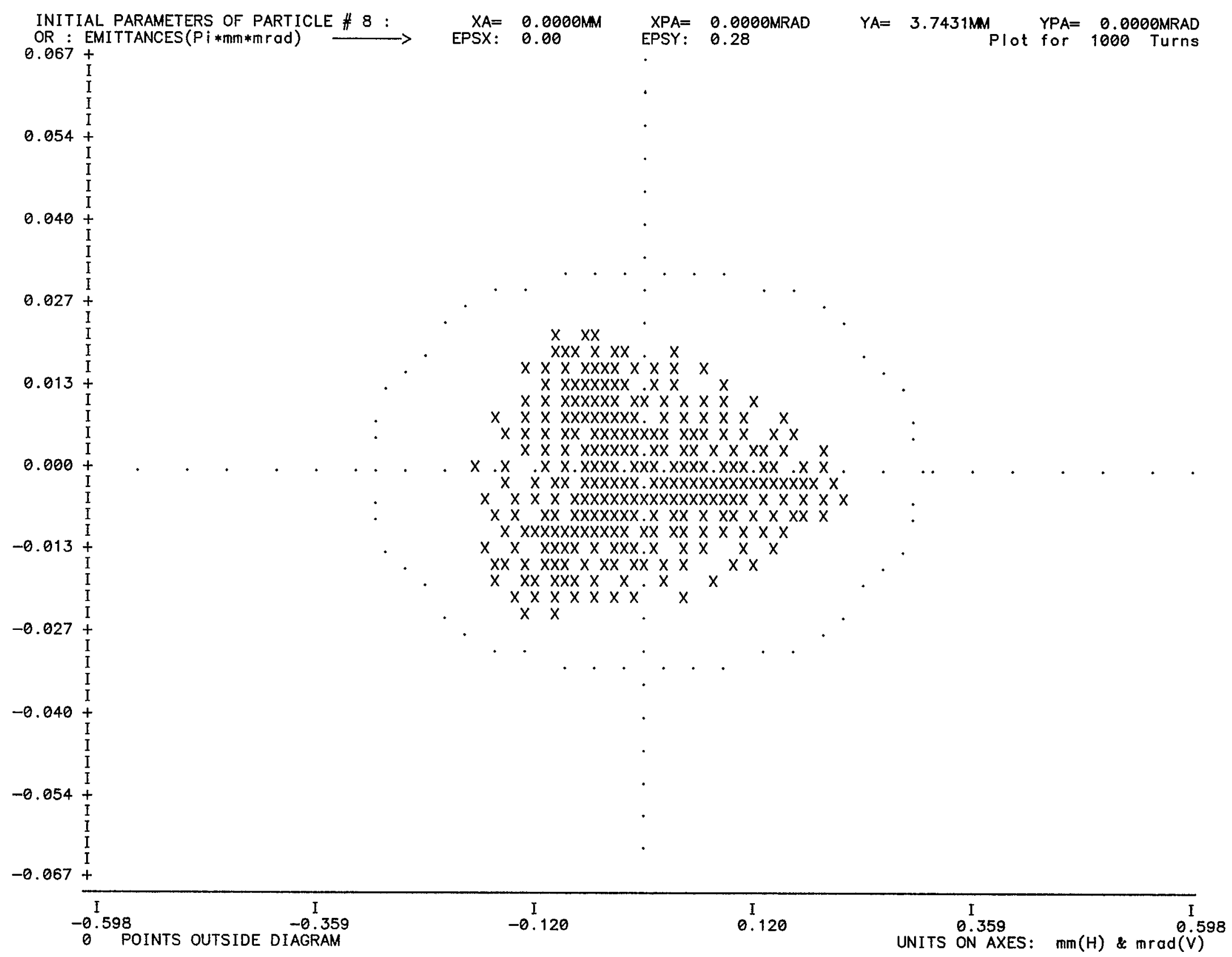

Figure 39. Particle trajectory in $\left(X, X^{\prime}\right)$ coordinates. Case \# 3 , particle no. 8 


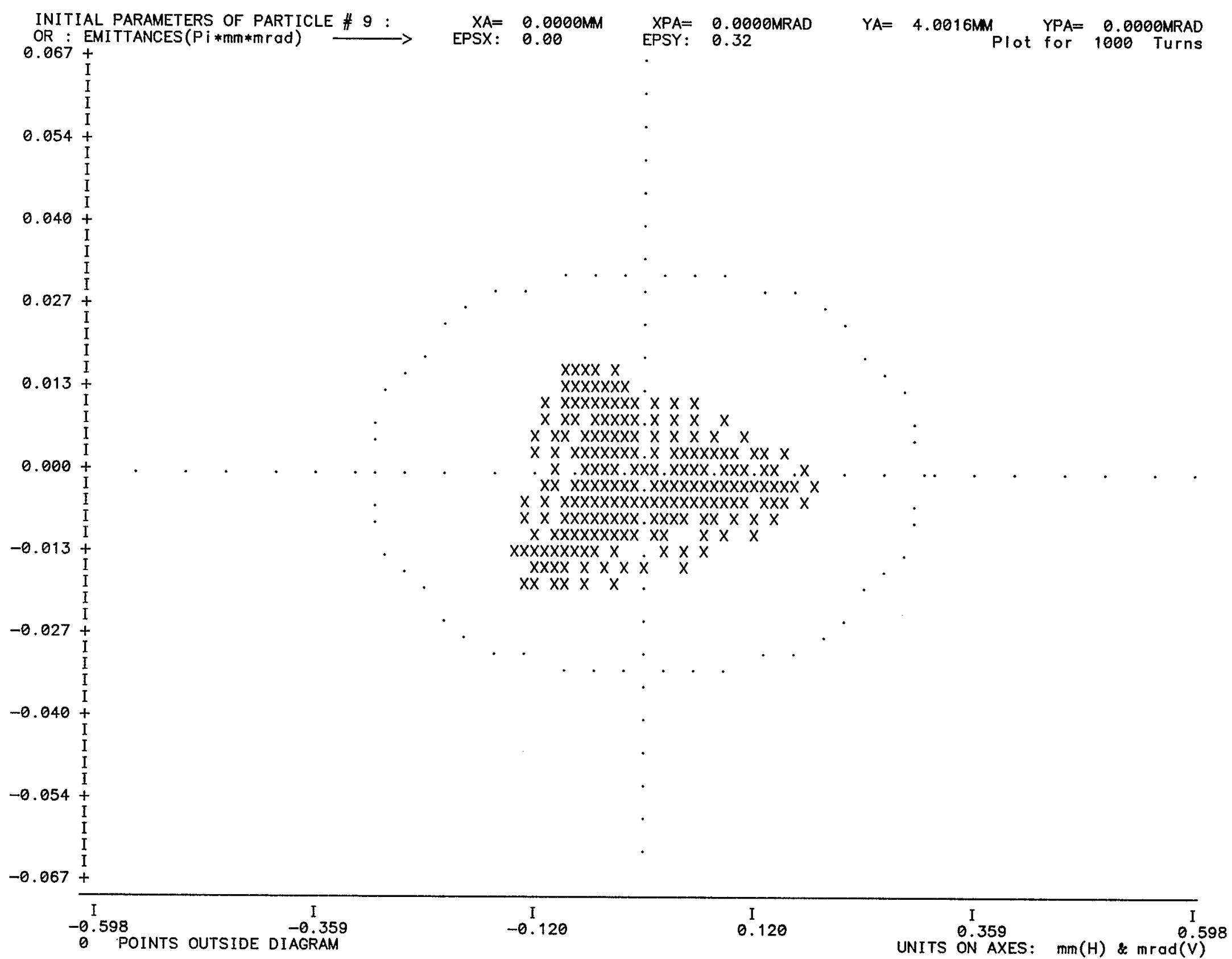

Figure 40. Particle trajectory in $\left(X, X^{\prime}\right)$ coordinates. Case \#3, particle no. 9 


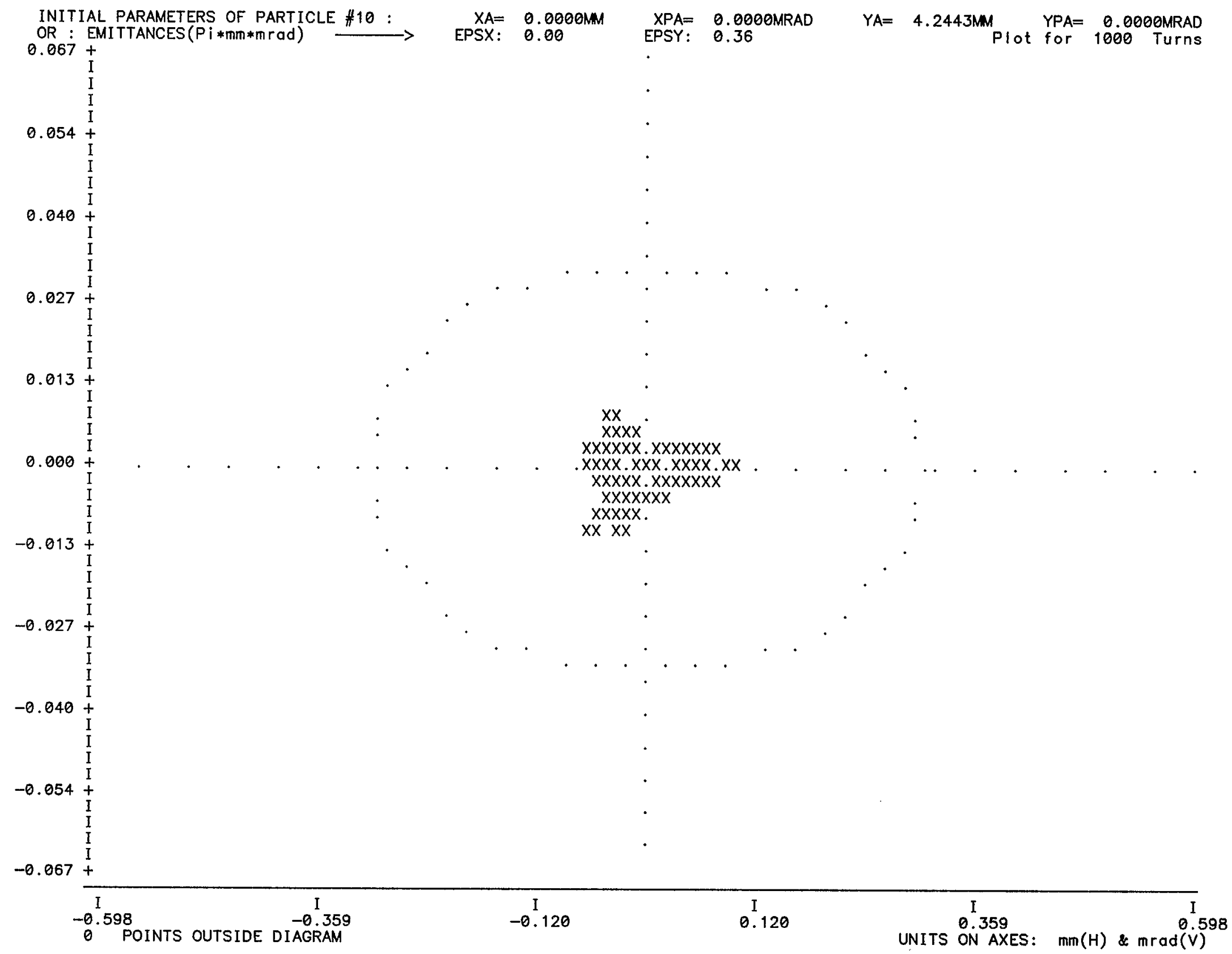

Figure 41. Particle trajectory in $\left(X, X^{\prime}\right)$ coordinates. Case \#3, particle no. 10 


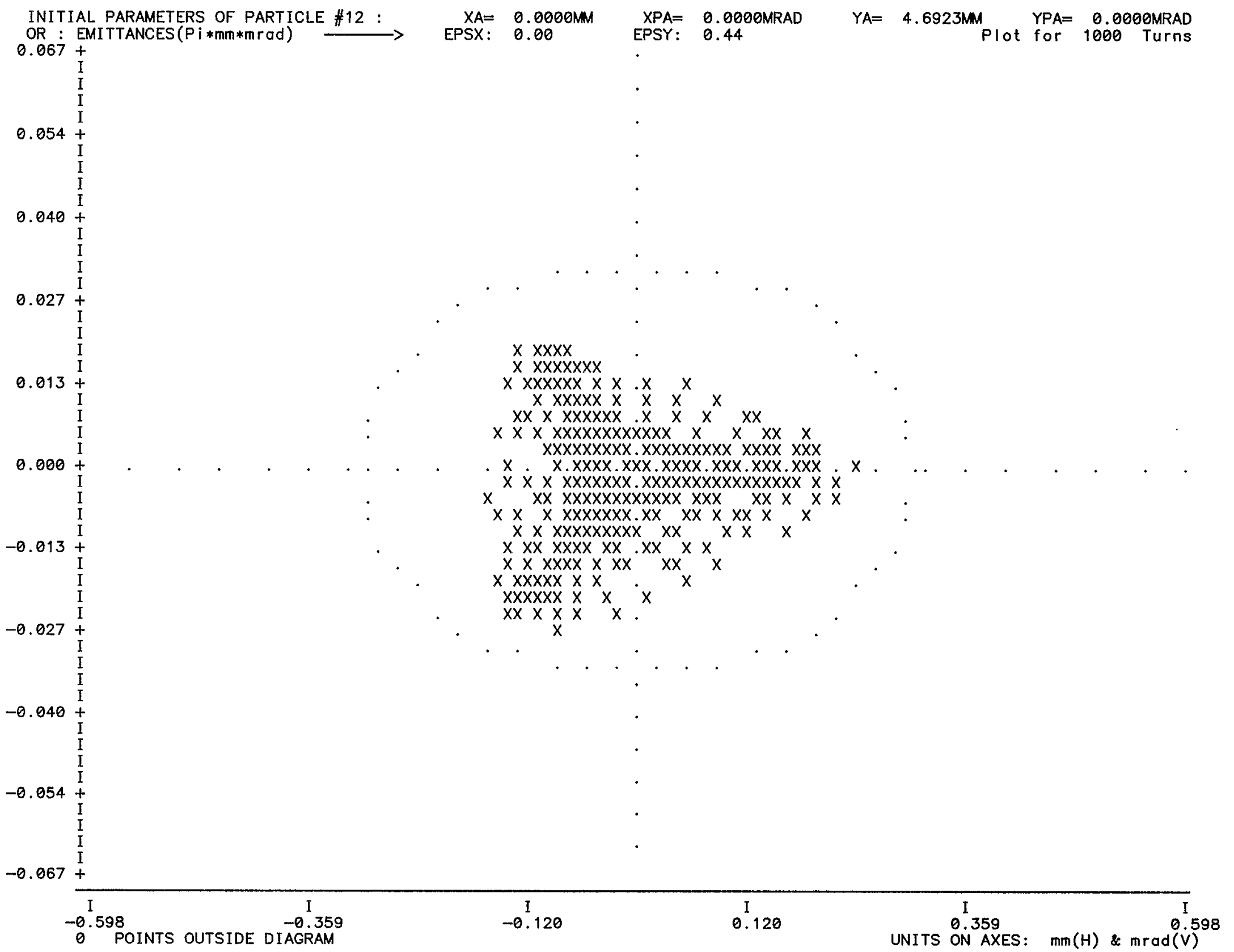

Figure 42. Particle trajectory in $\left(X, X^{\prime}\right)$ coordinates. Case \#3, particle no. 12 


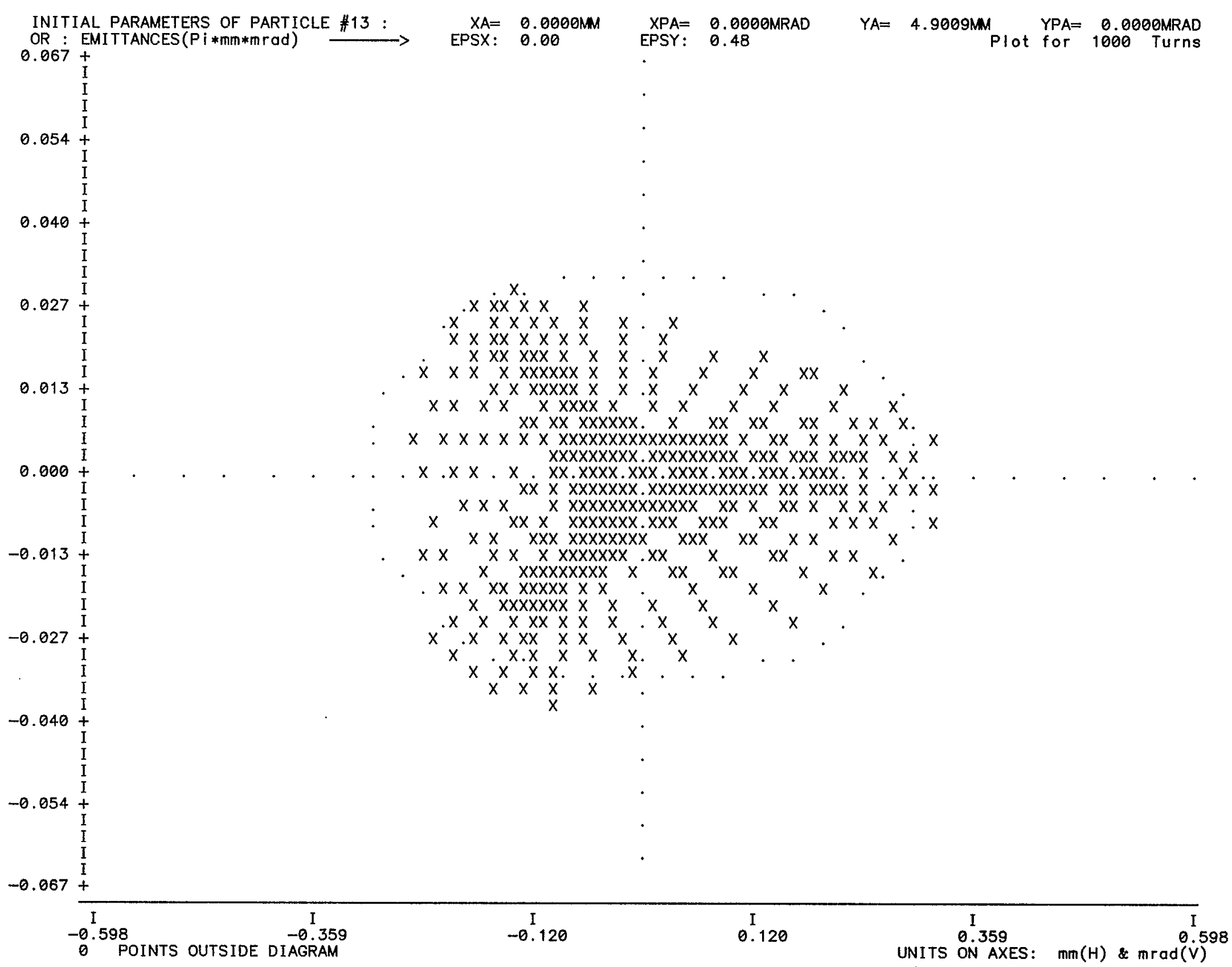

Figure 43. Particle trajectory in $\left(X, X^{\prime}\right)$ coordinates. Case \# 3, particle no. 13 


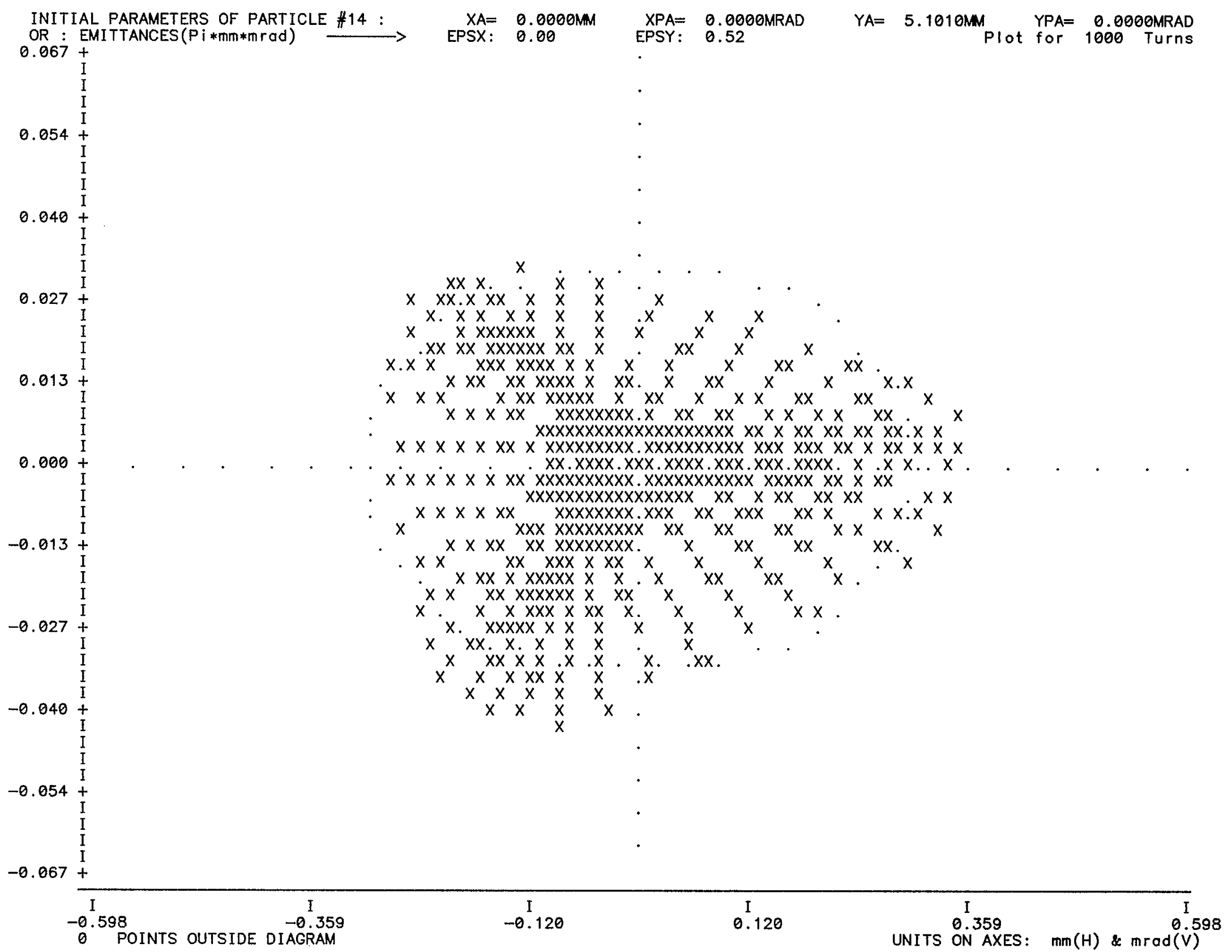

Figure 44. Particle trajectory in $\left(X, X^{\prime}\right)$ coordinates. Case \# 3 , particle no. 14 


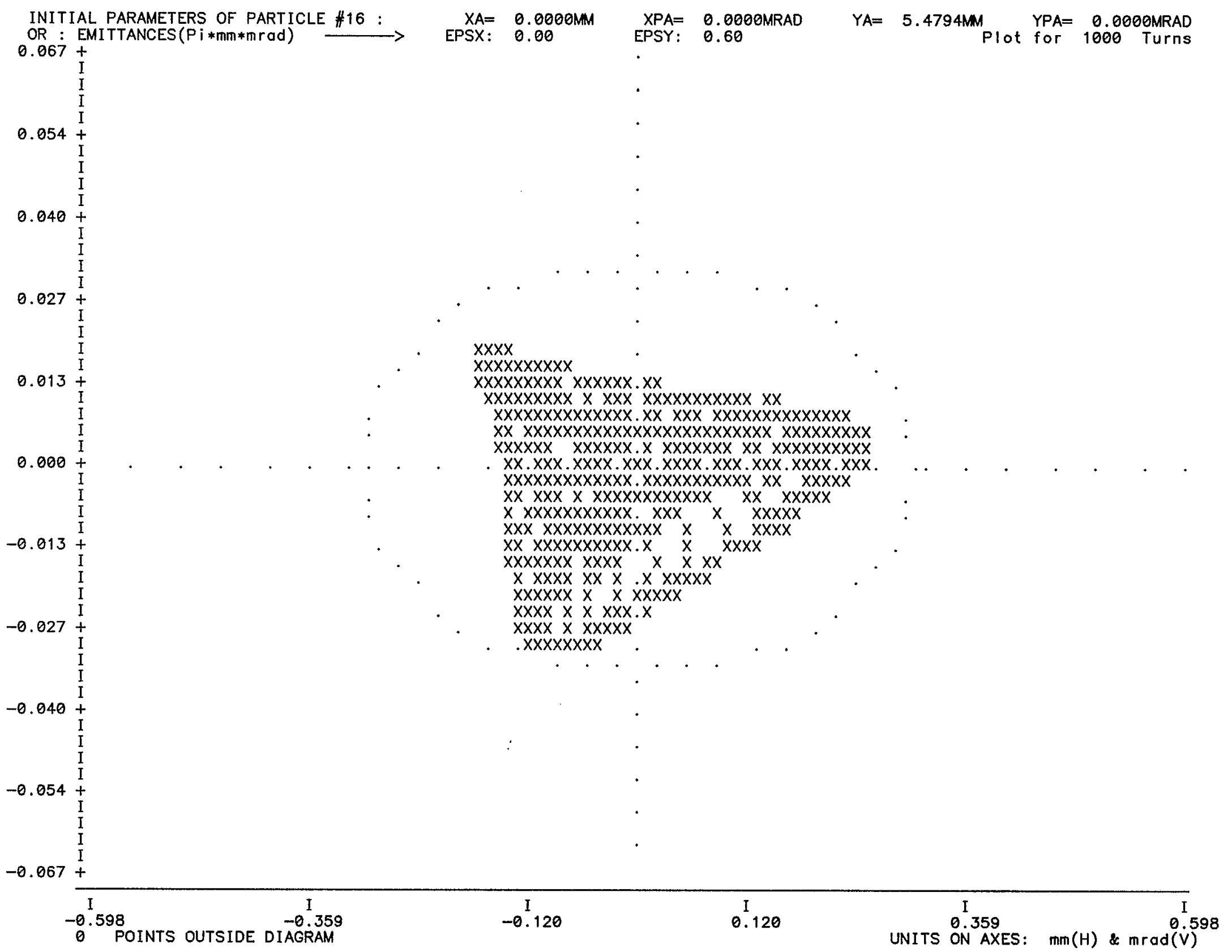

Figure 45. Particle trajectory in ( $\left.X, X^{\prime}\right)$ coordinates. Case $\#$ 3, particle no. 16 


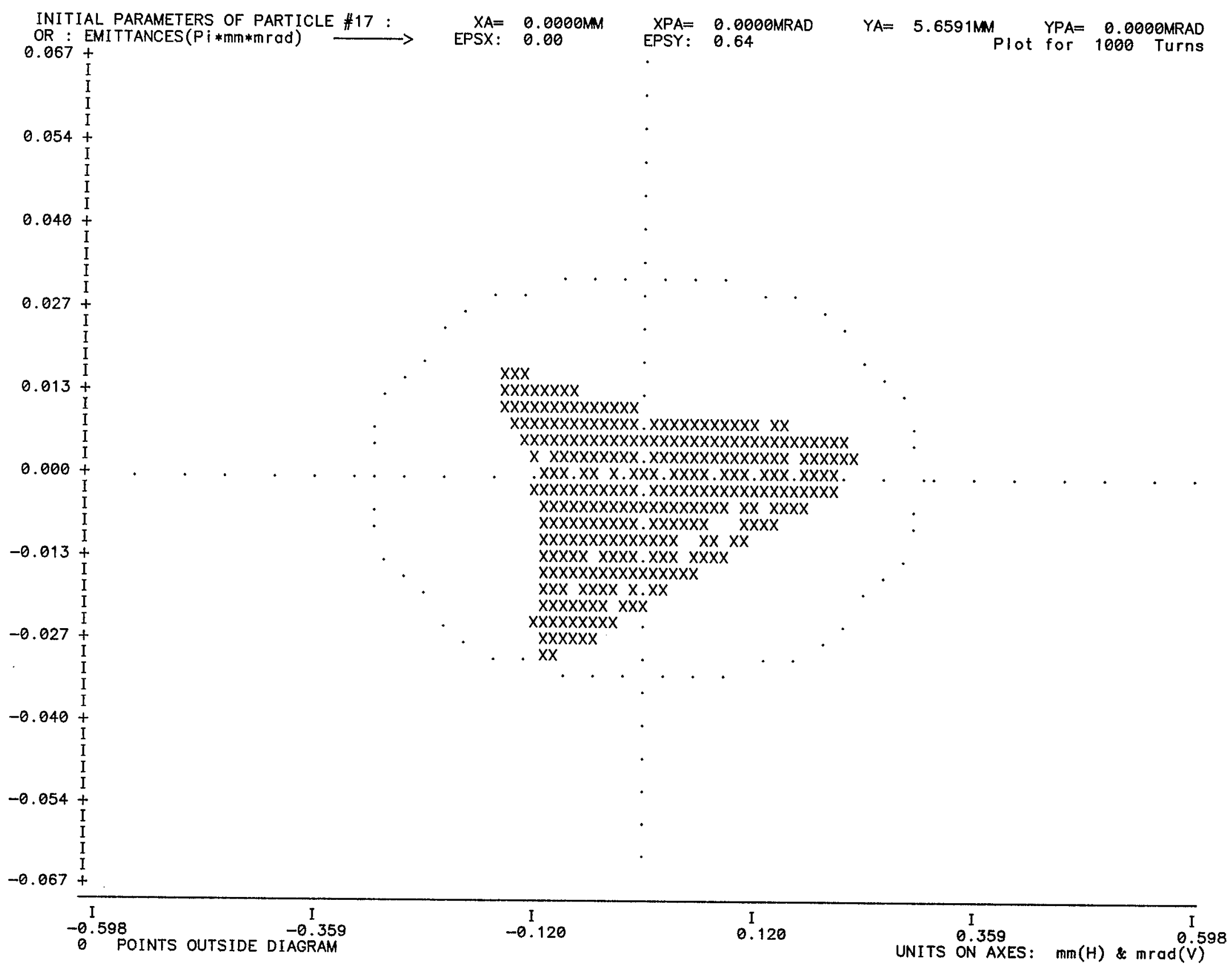

Figure 46. Particle trajectory in $\left(X, X^{\prime}\right)$ coordinates. Case \# 3, particle no. 17 


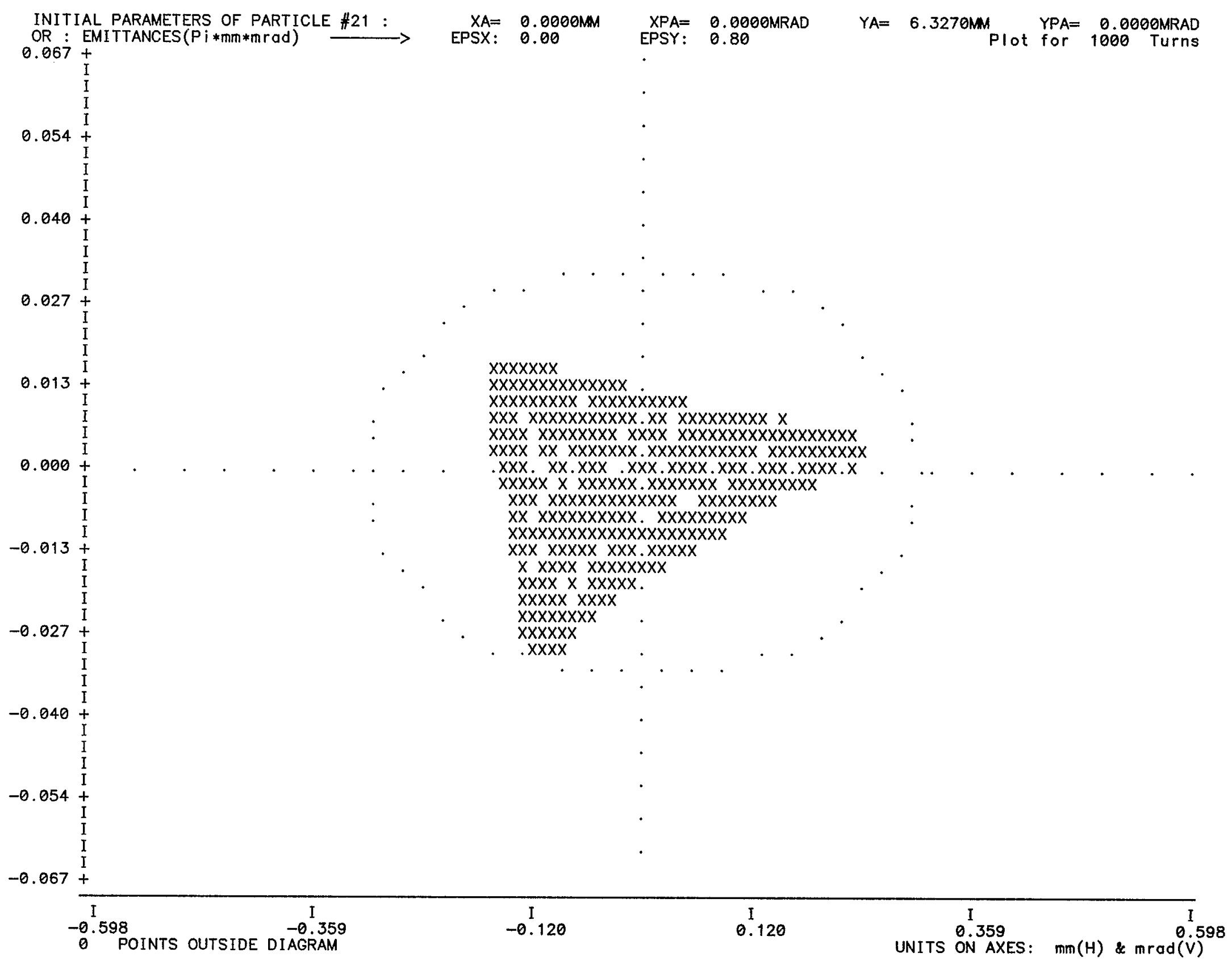

Figure 47. Particle trajectory in $\left(X, X^{\prime}\right)$ coordinates. Case \#3, particle no. 21 


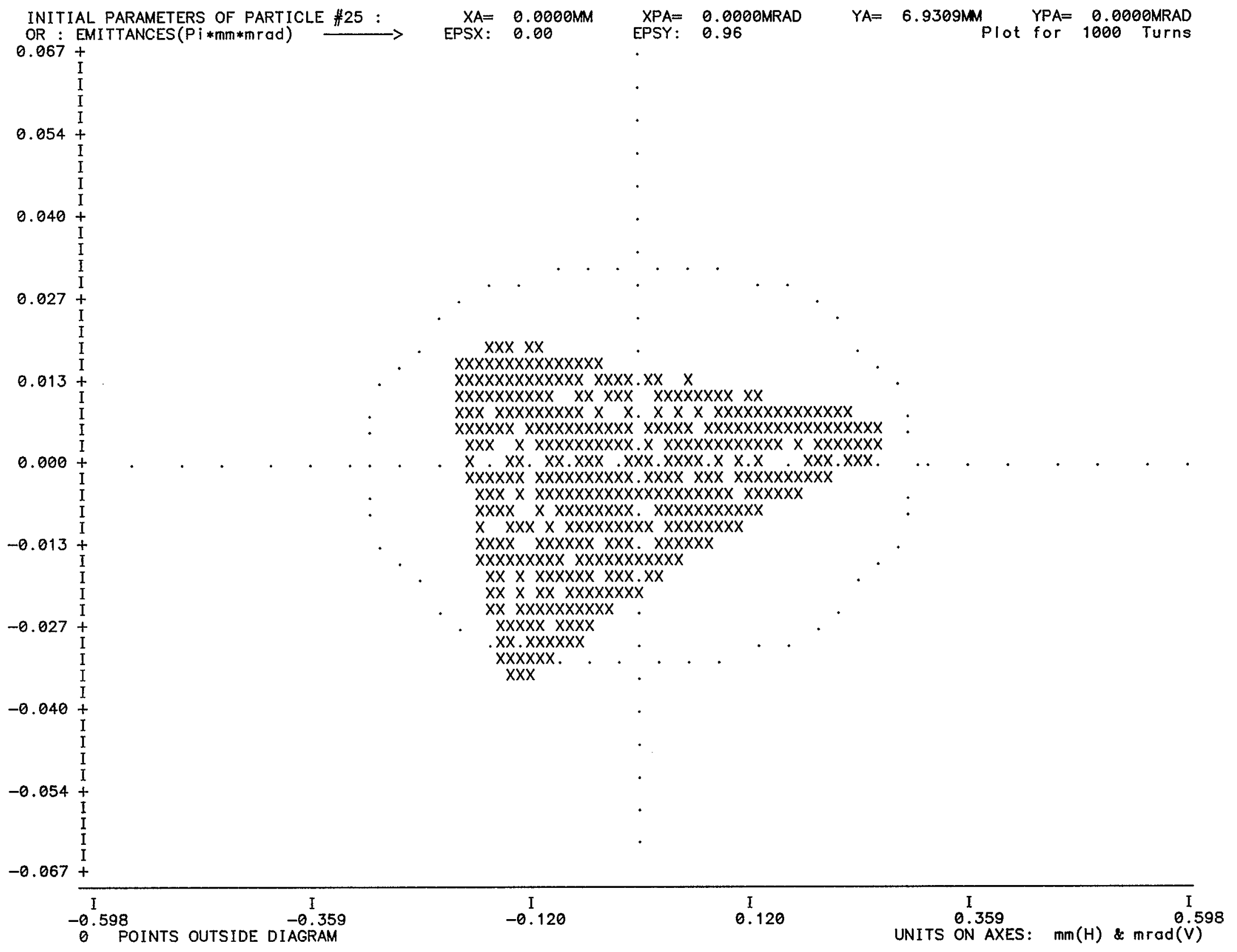

Figure 48. Particle trajectory in $\left(X, X^{\prime}\right)$ coordinates. Case \#3, particle no. 25 


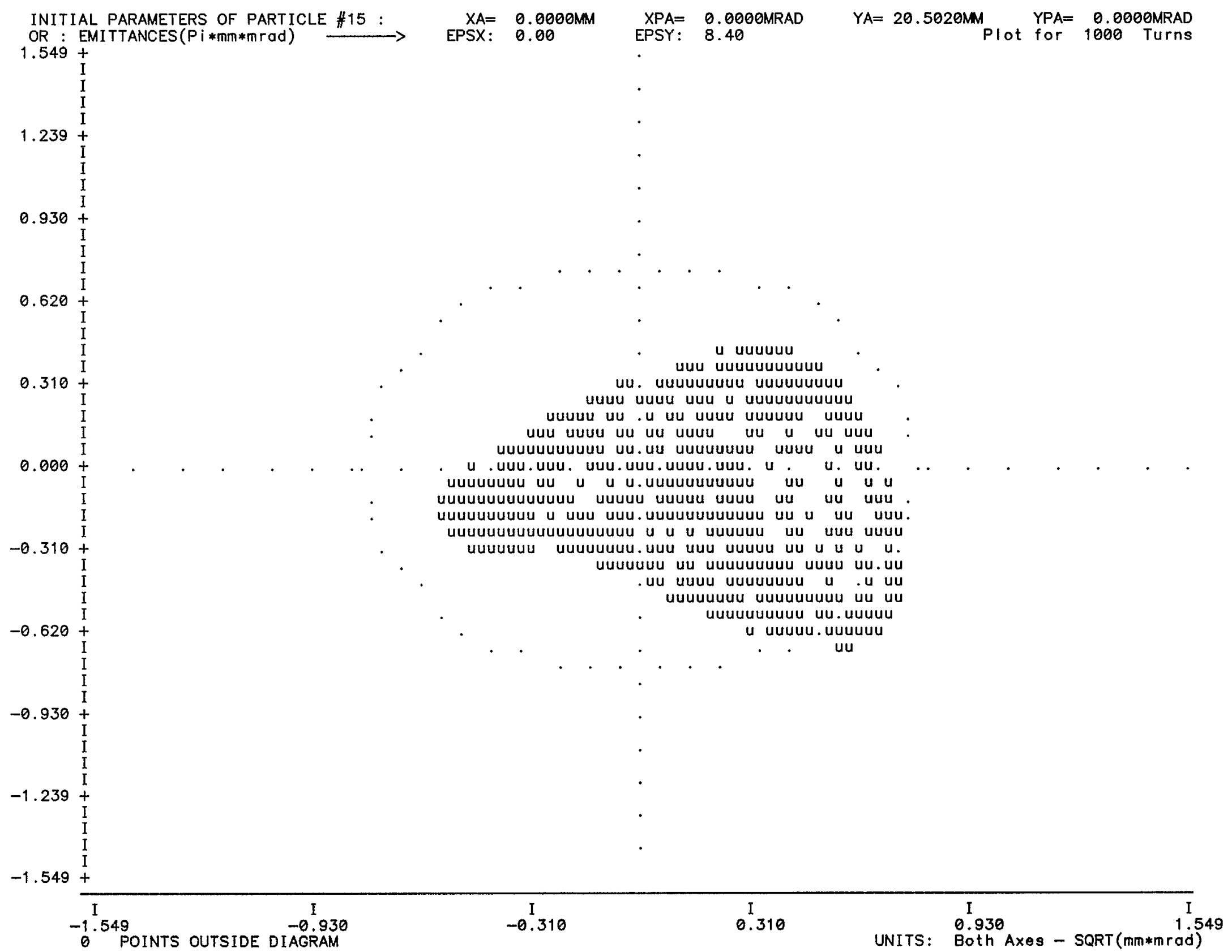

Figure 50. Particle trajectory in Floquet $\left(u, u^{\prime}\right)$ coordinates. Case \# 3 , large amplitude. 


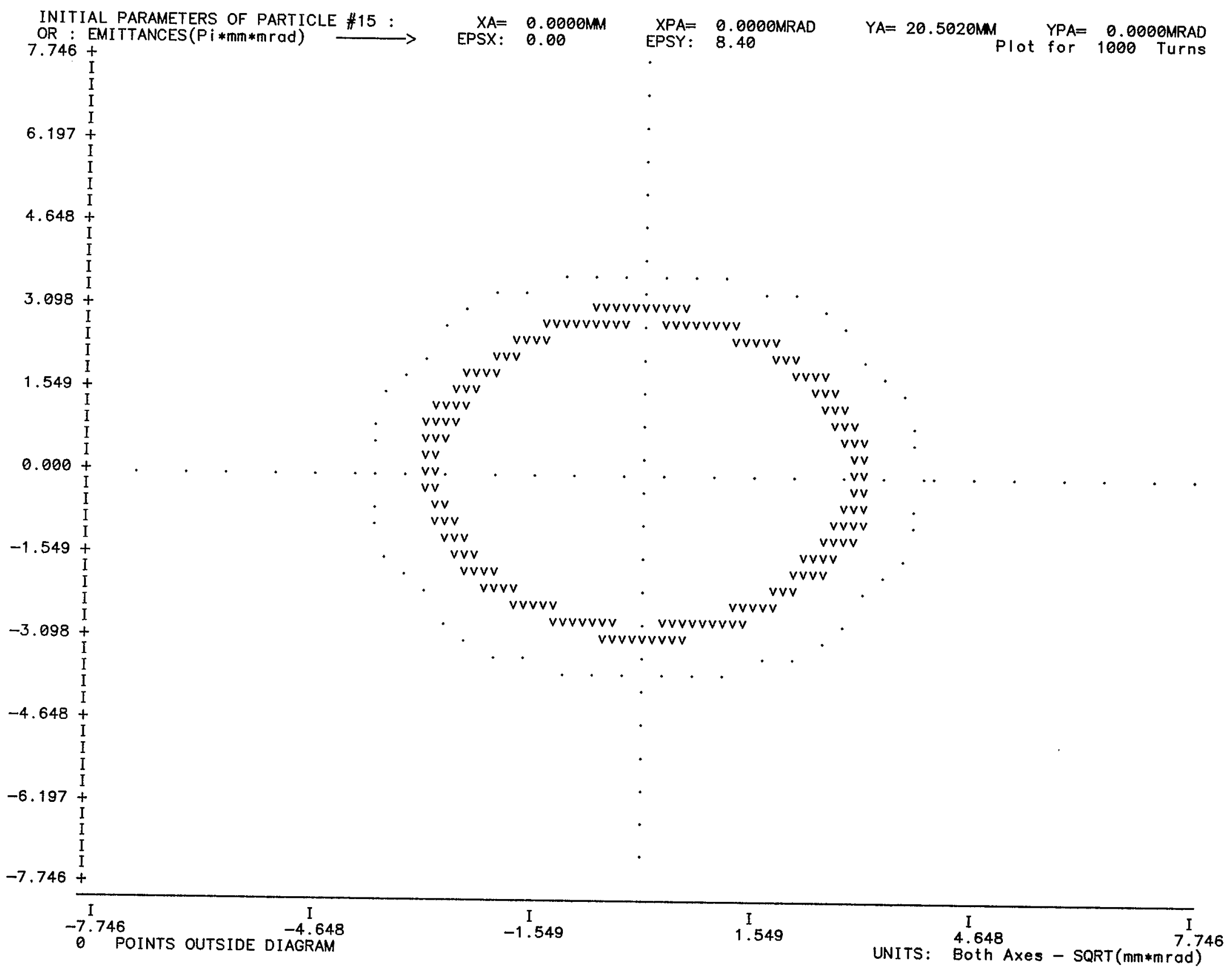

Figure 51. Particle trajectory in Floquet ( $\left.v, v^{\prime}\right)$ coordinates. Case \#3, large amplitude. 


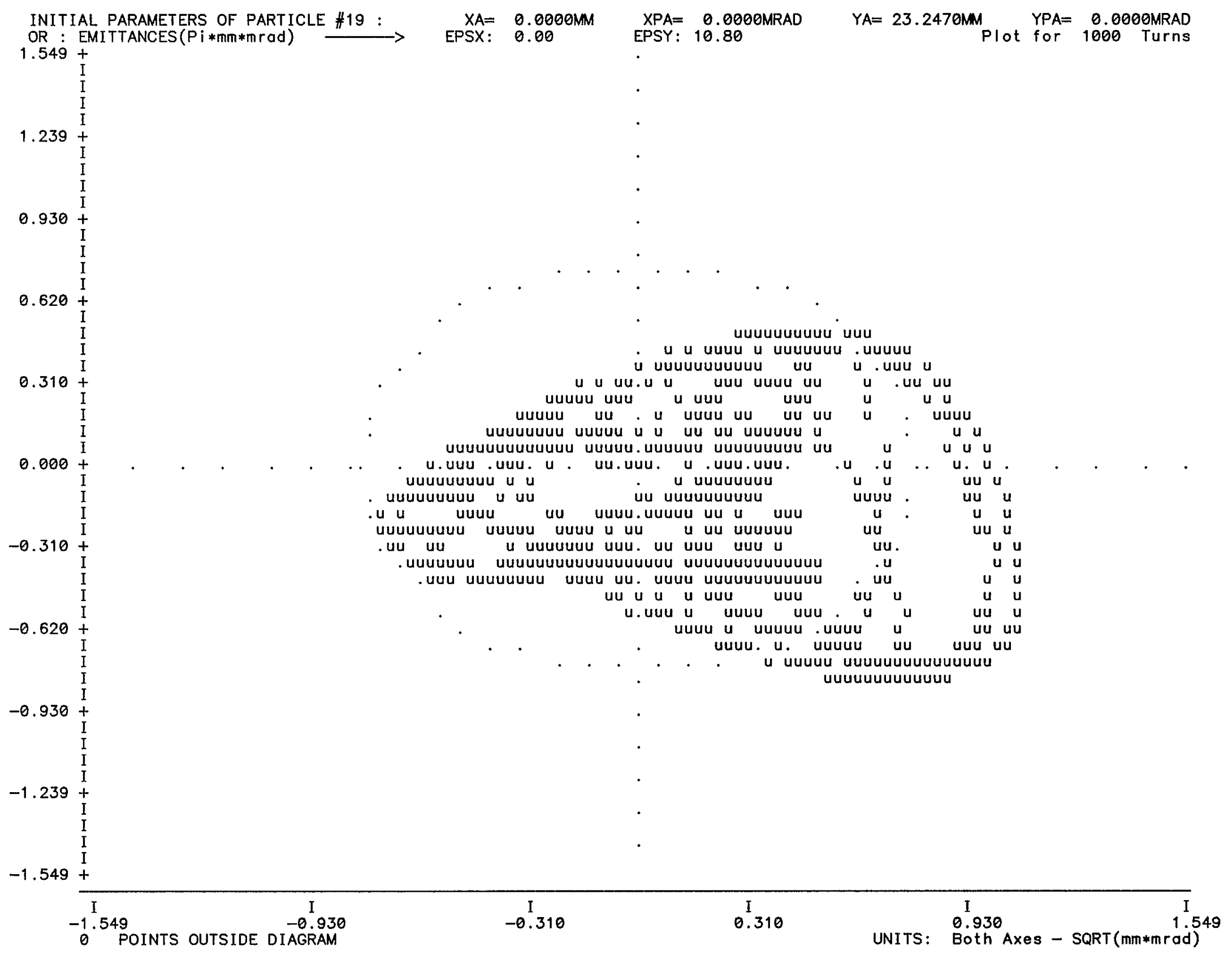

Figure 52. Particle trajectory in Floquet (u, $u^{\prime \prime}$ ) coordinates. Case \#3, amplitude close to dynamic aperture. 


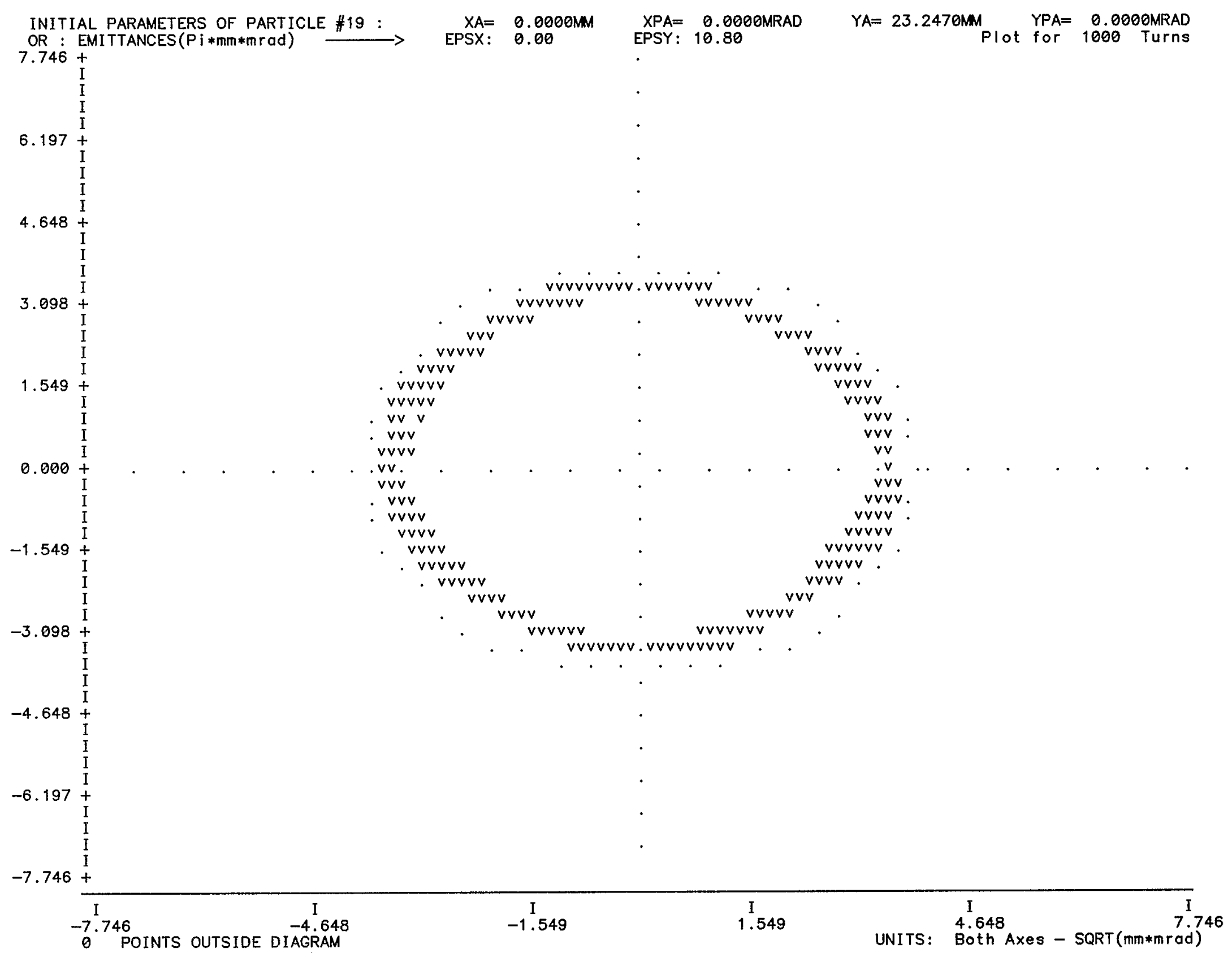

Figure 53. Particle trajectory in Floquet $\left(v, v^{\prime}\right)$ coordinates. Case $\# 3$, amplitude close to dynamic aperture. 


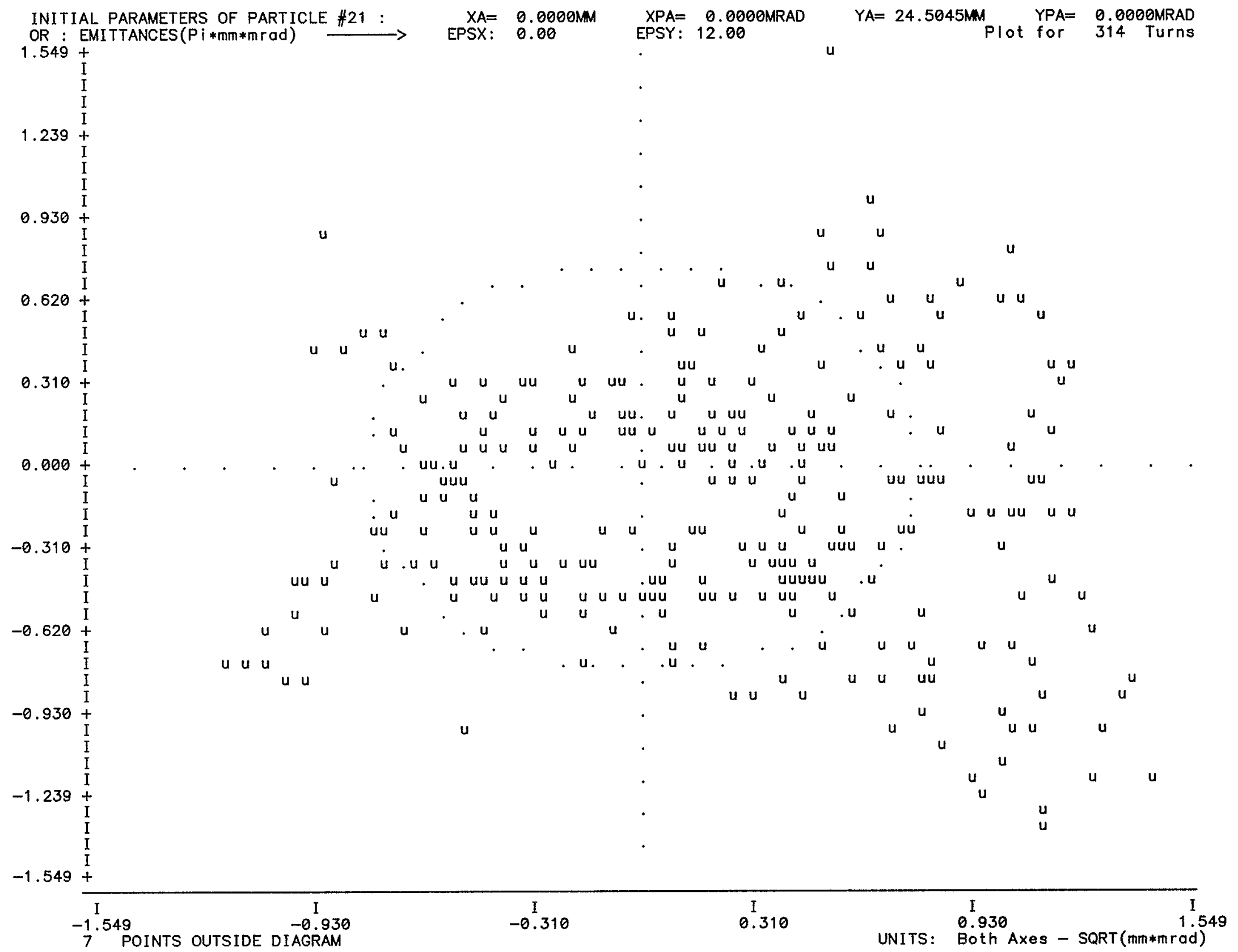

Figure 54. Particle trajectory in Floquet (u, $\left.u^{\prime}\right)$ coordinates. Case \# 3 , amplitude just outside dynamic aperture. 


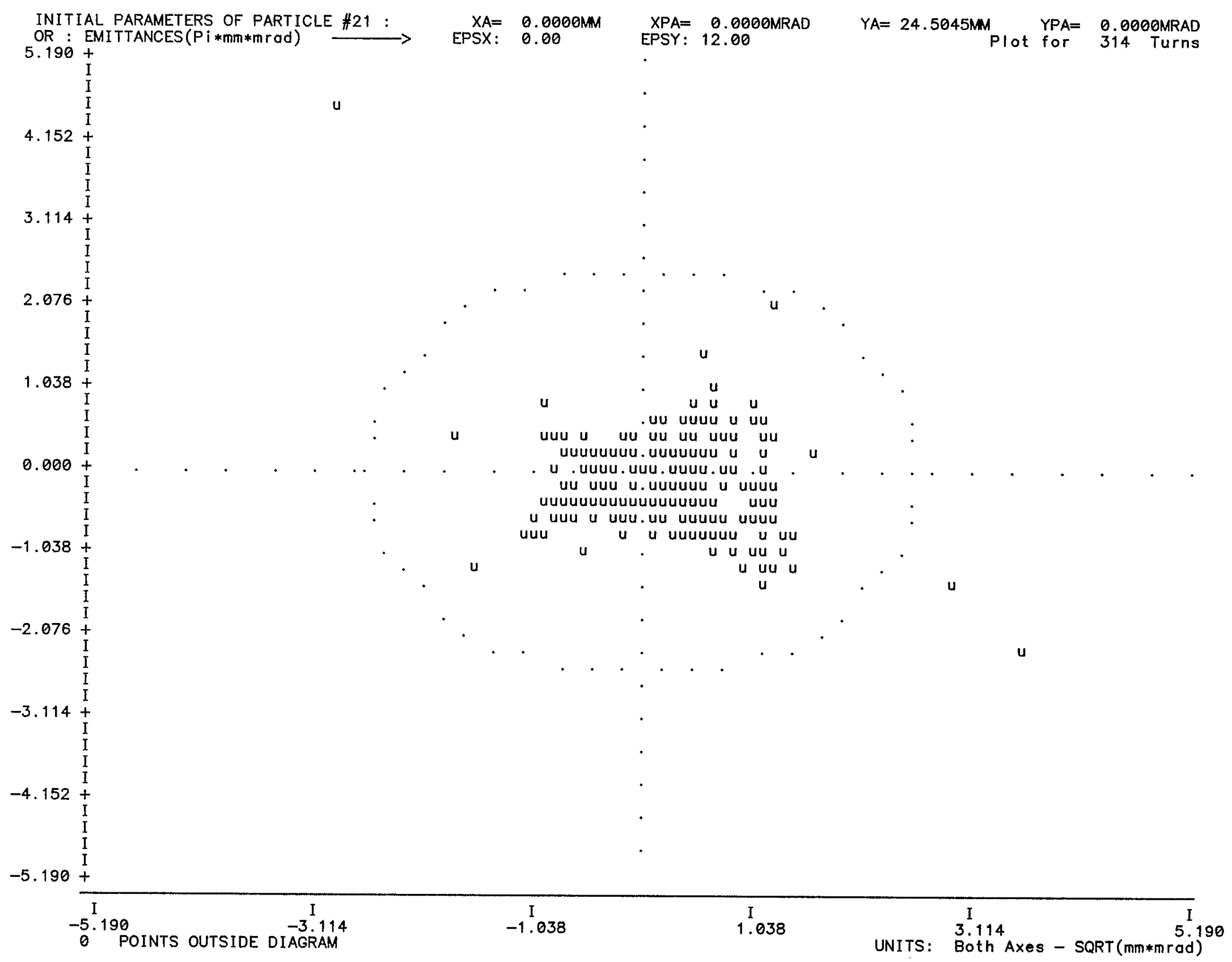

Figure 55. Particle trajectory in (u, $\left.u^{\prime}\right)$ coordinates. Case \# 3 , amplitude just outside dynamic aperture (larger scale). 


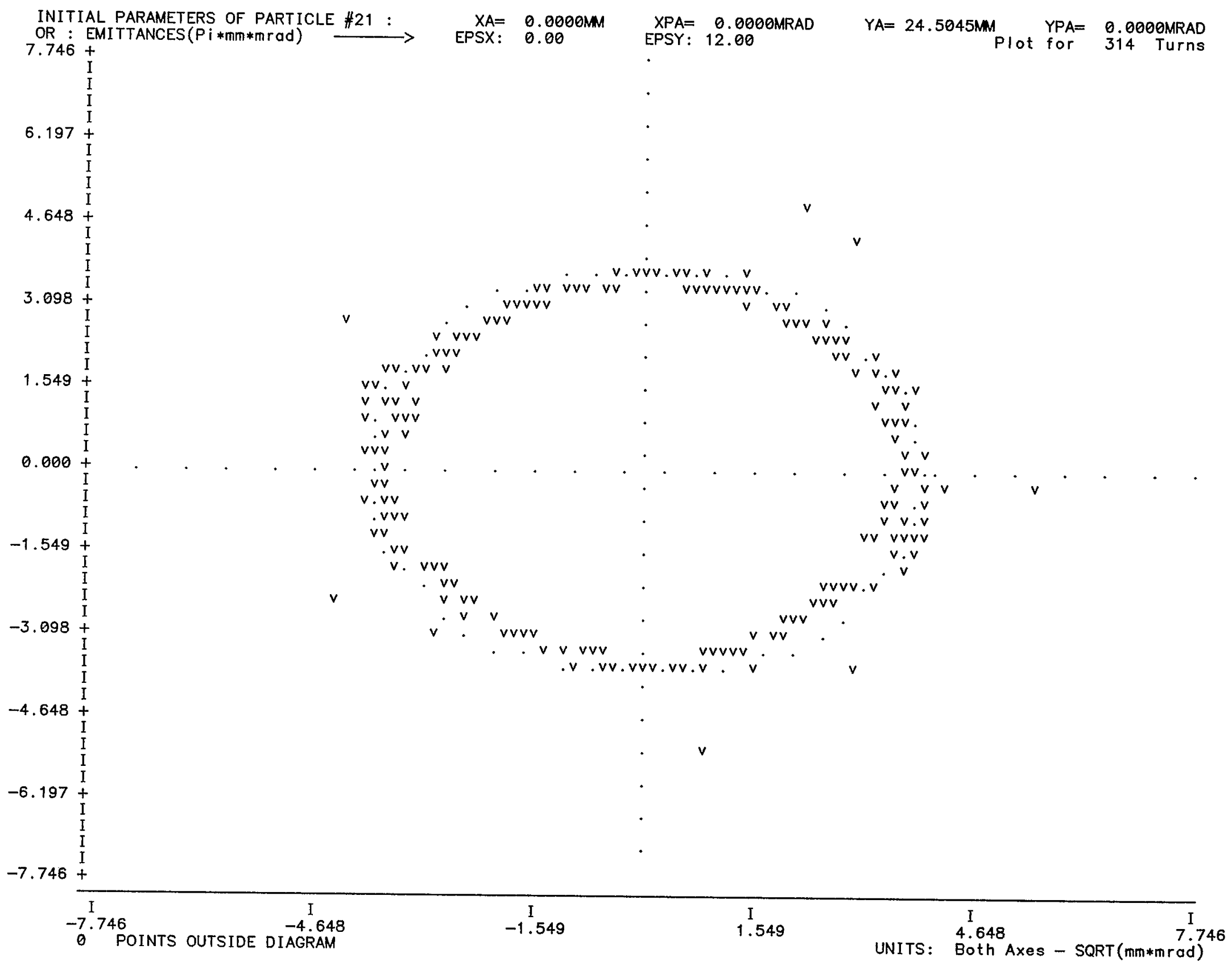

Figure 56. Particle trajectory in Floquet $\left(v, v^{\prime}\right)$ coordinates. Case \#3, amplitude just outside dynamic aperture. 